DOI 10.4171/JEMS/235

Pavel Etingof · Victor Ginzburg

\title{
Noncommutative del Pezzo surfaces and Calabi-Yau algebras
}

Received October 1, 2007 and in revised form February 22, 2010

Abstract. The hypersurface in $\mathbb{C}^{3}$ with an isolated quasi-homogeneous elliptic singularity of type $\widetilde{E}_{r}, r=6,7,8$, has a natural Poisson structure. We show that the family of del Pezzo surfaces of the corresponding type $E_{r}$ provides a semiuniversal Poisson deformation of that Poisson structure.

We also construct a deformation-quantization of the coordinate ring of such a del Pezzo surface. To this end, we first deform the polynomial algebra $\mathbb{C}\left[x_{1}, x_{2}, x_{3}\right]$ to a noncommutative algebra with generators $x_{1}, x_{2}, x_{3}$ and the following three relations labeled by cyclic parmutations $(i, j, k)$ of $(1,2,3)$ :

$$
x_{i} x_{j}-t \cdot x_{j} x_{i}=\Phi_{k}\left(x_{k}\right), \quad \Phi_{k} \in \mathbb{C}\left[x_{k}\right] .
$$

This gives a family of Calabi-Yau algebras $\mathfrak{A}^{t}(\Phi)$ parametrized by a complex number $t \in \mathbb{C}^{\times}$and a triple $\Phi=\left(\Phi_{1}, \Phi_{2}, \Phi_{3}\right)$ of polynomials of specifically chosen degrees.

Our quantization of the coordinate ring of a del Pezzo surface is provided by noncommutative algebras of the form $\mathfrak{A}^{t}(\Phi) /\left\langle\langle\Psi\rangle\right.$, where $\left\langle\langle\Psi\rangle \subset \mathfrak{A}^{t}(\Phi)\right.$ stands for the ideal generated by a central element $\Psi$ which generates the center of the algebra $\mathfrak{A}^{t}(\Phi)$ if $\Phi$ is generic enough.

\section{Contents}

1. Introduction . . . . . . . . . . . . . . . . . . . . . . . . . 1371

2. Poisson deformations of a quasi-homogeneous surface singularity $\ldots \ldots \ldots$. . . . 1377

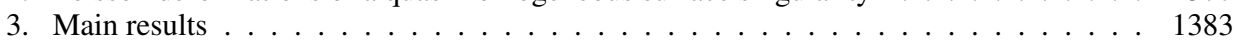

4. Three-dimensional Poisson structures . . . . . . . . . . . . . . . . . . . . 1391

5. Poisson (co)homology . . . . . . . . . . . . . . . . . . . . . . . . . . 1393

6. Classification results . . . . . . . . . . . . . . . . . . . . . . . . . . . . . . . . . . . . . . . . . 1400

7. Calabi-Yau deformations . . . . . . . . . . . . . . . . . . . . . . . . . . . . 1401

8. From Poisson to Hochschild cohomology . . . . . . . . . . . . . . . . . . . . . . . 1406

9. Appendix: computer calculation $\quad \ldots \ldots \ldots$. . . . . . . . . . . . . . . . . . . . . . . 1414

\section{Introduction}

\subsection{Poisson structures on del Pezzo surfaces}

We remind the reader that a del Pezzo surface is a smooth projective surface $S$ that is obtained by blowing up $\ell$ sufficiently general points in $\mathbb{C P}^{2}$, where $0 \leq \ell \leq 8$, or $\mathbb{C P}^{1} \times \mathbb{C P}^{1}$.

P. Etingof: Department of Mathematics, Massachusetts Institute of Technology, Cambridge, MA 02139, USA; e-mail: etingof@ math.mit.edu

V. Ginzburg: Department of Mathematics, University of Chicago, Chicago, IL 60637, USA; e-mail: ginzburg@math.uchicago.edu 
Let $S$ be such a del Pezzo surface with canonical bundle $K_{S}$, resp. anticanonical bundle $K_{S}^{-1}$. A regular section $\pi \in \Gamma\left(S, K_{S}^{-1}\right)$ is a bivector that gives $S$ a Poisson structure (any bivector $\pi$ on a surface automatically has a vanishing Schouten bracket: $[\pi, \pi]=0$ ). We say that a regular section $\pi \in \Gamma\left(S, K_{S}^{-1}\right)$ is nondegenerate provided the divisor of zeros of $\pi$ is a reduced smooth curve.

In this paper we consider the most interesting case where $\ell=6,7$, or 8 , and where $\pi$ is assumed to be a nondegenerate section. Then a simple application of the adjunction formula shows that the zero locus of $\pi$ is an elliptic curve $\mathbb{E} \subset S$. Furthermore, $X:=$ $S \backslash \mathbb{E}$ is an affine surface equipped with an algebraic symplectic structure provided by the (closed) 2-form $\pi^{-1} \in \Gamma\left(S \backslash \mathbb{E}, K_{S}\right)$.

There are two Poisson algebras naturally associated with the data $(S, \pi)$. The first algebra is $\mathbb{C}[X]$, the coordinate ring of the affine symplectic surface $X$. The second algebra is a graded algebra

$$
\mathcal{R}=\bigoplus_{n \geq 0} \mathcal{R}_{n}, \quad \mathcal{R}_{n}:=\Gamma\left(S,\left(K_{S}^{-1}\right)^{\otimes n}\right),
$$

the homogeneous coordinate ring associated with the anticanonical bundle, an ample line bundle on $S$. One can use a construction of Kaledin to make $\mathcal{R}$ a Poisson algebra as follows.

Choose a local nowhere vanishing section $\phi \in \Gamma\left(U, \mathcal{K}_{S}^{-1}\right)$ on a Zariski open subset $U \subset S$. Let $L_{h}(\phi):=\left[i_{\pi}(d h), \phi\right]$ denote the Lie derivative of the bivector $\phi$ with respect to $i_{\pi}(d h)$, the Hamiltonian vector field associated with a regular function $h$ on $U$. Further, write $\{-,-\}_{\pi}$ for the Poisson bracket on $U$ induced by the bivector $\pi$. Then, following $\lceil\mathrm{Ka}]$, one defines a Poisson bracket $\{-,-\}_{\mathcal{R}}: \mathcal{R}_{n} \times \mathcal{R}_{m} \rightarrow \mathcal{R}_{n+m}, m, n \geq 0$, by the formula

$\left\{f \phi^{n}, g \phi^{m}\right\}_{\mathcal{R}}:=\{f, g\}_{\pi} \cdot \phi^{n+m}+\left(m g L_{f}(\phi)-n f L_{g}(\phi)\right) \cdot \phi^{n+m-1}, \quad \forall f, g \in \Gamma\left(U, \mathcal{O}_{U}\right)$.

It is straightforward to verify that the resulting bracket is independent of the choice of a nowhere vanishing section $\phi$ on $U$.

To relate the Poisson algebras $\mathbb{C}[X]$ and $\mathcal{R}$, write $K$ for the total space of the canonical bundle $K_{S}$. Thus $K$ is a 3-dimensional variety equipped with a natural $\mathbb{C}^{\times}$-action. By definition, one has a graded algebra isomorphism $\mathcal{R}=\mathbb{C}[K]:=\Gamma\left(K, \mathcal{O}_{K}\right)$ such that the grading on $\mathbb{C}[K]$ comes from the $\mathbb{C}^{\times}$-action. Further, there is a diagram

$$
X=S \backslash \mathbb{E} \stackrel{i:=\pi^{-1}}{\longrightarrow} K \stackrel{p}{\rightarrow} S,
$$

where the second map $p$ is the line bundle projection and the first map is a section of $p$ over $S \backslash \mathbb{E}$ provided by the symplectic form.

One can show that the map $i=\pi^{-1}$, in the diagram, is a closed imbedding. Moreover, the corresponding restriction morphism $i^{*}: \mathcal{R}=\mathbb{C}[K] \rightarrow \mathbb{C}[X]$ induces an algebra isomorphism $\mathcal{R} /(\pi-1) \stackrel{\sim}{\rightarrow} \mathbb{C}[X]$, where $(\pi-1)$ denotes the ideal generated by the element $\pi-1 \in \mathcal{R}_{1} \oplus \mathcal{R}_{0}$. The element $\pi-1$ being nonhomogeneous, the grading on the 
algebra $\mathcal{R}$ does not descend to a grading on the quotient algebra. However, the ascending filtration $F_{\leq m} \mathcal{R}:=\bigoplus_{n \leq m} \mathcal{R}_{m}$ on $\mathcal{R}$ induces a well-defined ascending filtration $F . \mathbb{C}[X]$ that makes the coordinate ring $\mathbb{C}[X]$ a filtered algebra.

Let $\mathrm{R} \mathbb{C}[X]:=\sum_{n \geq 0} F_{n} \mathbb{C}[X] \cdot t^{n} \subset \mathbb{C}[X] \otimes \mathbb{C}[t]$ be the Rees algebra of the filtered algebra $\mathbb{C}[X]$. This is a graded algebra equipped with a canonical graded algebra imbedding $\mathbb{C}[t] \hookrightarrow \mathrm{R} \mathbb{C}[X]$ such that $\mathrm{R} \mathbb{C}[X] /(t-1) \cong \mathbb{C}[X]$. Furthermore, the Poisson bracket on $\mathbb{C}[X]$ induces one on the Rees algebra.

We leave to the reader the proof of the following simple result:

Proposition 1.1.3. There is a natural graded Poisson algebra isomorphism $\Xi$ : $\mathrm{R} \mathbb{C}[X] \stackrel{\sim}{\rightarrow} \mathcal{R}$ such that

- The canonical algebra imbedding $\mathbb{C}[t] \hookrightarrow \mathrm{R} \mathbb{C}[X]$ gets transported, via $\Xi$, to the graded algebra homomorphism $\mathbb{C}[t] \hookrightarrow \mathcal{R}$ induced by the assignment $t \mapsto \pi$.

- The isomorphism $\mathrm{R} \mathbb{C}[X] /(t-1) \cong \mathbb{C}[X]$ gets transported, via $\Xi$, to the algebra isomorphism $\mathbb{C}[K] /(\pi-1) \stackrel{\sim}{\rightarrow} \mathbb{C}[X]$.

The proposition shows how the Poisson algebras $\mathbb{C}[X]$ and $\mathcal{R}$ can be recovered from each other. Therefore, quantization (i.e., noncommutative deformation) problems for these two algebras are essentially equivalent.

In the rest of the paper, we will concentrate on the problem of quantizing the affine symplectic surface $X=S \backslash \mathbb{E}$ by constructing noncommutative deformations of the Poisson algebra $\mathbb{C}[X]$, to be viewed as coordinate rings of 'noncommutative affine surfaces'. Noncommutative deformations of the Poisson algebra $\mathcal{R}$, to be viewed as homogeneous rings of 'noncommutative projective surfaces', may then be obtained by applying the Rees algebra construction to the corresponding noncommutative deformations of $\mathbb{C}[X]$.

\subsection{Noncommutative surfaces}

The general theory of noncommutative projective surfaces has been initiated in the late 80's by Artin and Schelter [AS]. Many deep results were obtained later, in the papers [ATV], [AV], [BSV], [C1], [Le], and [St1].

The general philosophy of noncommutative surfaces, either projective or affine, was outlined by M. Artin in $[\mathrm{A}]$. According to that philosophy in the affine case, one tries to construct a noncommutative algebra $B$ that plays the role of 'coordinate ring' of an (affine) noncommutative surface $X$. It turns out that, in typical examples, the algebra $B$ often appears in the form $B=A /\langle\langle\Psi\rangle$. Here, $A$ is an auxiliary associative algebra which is somehow more accessible than $B$, and $\langle\langle\Psi\rangle\rangle$ denotes a two-sided ideal in $A$ generated by a normal (often central) element $\Psi \in A$. It has been remarked by M. Artin [A] that there should be some more conceptual a priori explanation of the appearance of the algebra $A$ and of the element $\Psi$.

The aim of the present paper is to propose such an explanation. Our approach is based on the concept of Calabi-Yau (CY) algebra, introduced recently (cf. e.g. [Bo], [Gi], and Definition 1.4.1 below). This approach is consistent with the point of view of string 
theory where 3-dimensional CY varieties are considered to be more fundamental than 2dimensional surfaces. Thus, a 2-dimensional surface should be viewed as a hypersurface in an ambient CY 3-fold which, in the affine case, is typically taken to be $\mathbb{C}^{3}$ and, in the projective case, is taken to be the total space of the canonical bundle of the surface.

The best way to understand what kind of noncommutative algebraic structures should be analogous to the structures of CY geometry is to consider a 'quasi-classical approximation' first. A noncommutative CY algebra of dimension 3 reduces, quasi-classically, to the coordinate ring $\mathbb{C}[M]$ of an affine 3-dimensional variety $M$. Such a variety comes equipped with an algebraic volume form vol $\in \Omega^{3}(M)$, that keeps track of the CY structure, and with a Poisson bracket, that 'remembers' about the noncommutative deformation, up to first order. A key point is that these two pieces of data must be related. Specifically, it was explained by Dolgushev [Do] that the correct quasi-classical analogue of the $\mathrm{CY}$ condition is the requirement that the Poisson bracket on $M$ be unimodular, that is, such that any Hamiltonian vector field on $M$ preserves the volume form vol, i.e. has the vanishing divergence.

It is easy to show that any unimodular Poisson bracket on a 3-fold with trivial first de Rham cohomology is determined by a single regular function $\phi \in \mathbb{C}[M]$ (see $\$ 4$ ). The function $\phi$ is unique up to a constant summand and it is automatically central with respect to the corresponding Poisson bracket. Furthermore, this function generates, generically, the whole Poisson center.

We turn now to noncommutative surfaces inside our noncommutative CY variety. Quasi-classically, giving such a surface amounts to giving a Poisson hypersurface $X \subset M$. For $M=\mathbb{C}^{3}$, for instance, that means, in the generic case, that the equation of the hypersurface $X$ must be given by a function contained in the Poisson center of $\mathbb{C}[M]$. In the situation where the Poisson center reduces to $\mathbb{C}[\phi]$ we conclude that our function is a polynomial in $\phi$. Hence, the only hypersurfaces which may arise in the process of quasiclassical degeneration of a noncommutative story are, essentially, the level sets of $\phi$. By redefining $\phi$, one may assume without loss of generality that the surface is the zero set of $\phi$, so the corresponding coordinate ring is $\mathbb{C}[X]=\mathbb{C}[M] /(\phi)$.

The discussion above suggests that $\mathbb{C}[M]$, the coordinate ring of the CY 3-fold, gets deformed via a quantization to a noncommutative $\mathrm{CY}$ algebra $A$ in such a way that the function $\phi$ gets deformed to a central (more generally, normal) element $\Psi \in A$. Therefore, the coordinate ring of the corresponding surface gets deformed to a noncommutative algebra of the form $B=A /\langle\langle\Psi\rangle\rangle$.

This provides a reason for the appearance of the objects $A$ and $\Psi$ we were looking for.

\subsection{Quantizing del Pezzo surfaces}

In this paper, we study hypersurfaces in the $\mathrm{CY}$ variety $M=\mathbb{C}^{3}$, equipped with the standard volume form $\mathrm{d} x \wedge \mathrm{d} y \wedge \mathrm{d} z$. Thus, we have $\mathbb{C}[M]=\mathbb{C}[x, y, z]$. As we have mentioned earlier, associated with any $\phi \in \mathbb{C}[x, y, z]$, there is a Poisson structure on $M$. Specifically, the Poisson brackets of coordinate functions are given by the explicit formulas

$$
\{x, y\}=\frac{\partial \phi}{\partial z}, \quad\{y, z\}=\frac{\partial \phi}{\partial x}, \quad\{z, x\}=\frac{\partial \phi}{\partial y} .
$$


It is immediate to verify that $\phi$ is a central element with respect to the above bracket. Therefore, $\mathbb{C}[x, y, z] /(\phi)$, a quotient by the principal ideal generated by $\phi$, inherits the structure of a Poisson algebra.

Definition 1.3.2. We write $\mathscr{A}_{\phi}:=\mathbb{C}[x, y, z]$ for the Poisson algebra with bracket (1.3.1), and let $\mathscr{B}_{\phi}:=\mathscr{A}_{\phi} /(\phi)$ be the quotient Poisson algebra with induced bracket.

It is interesting to take $\phi$ a (quasi-) homogeneous polynomial with an isolated singularity at the origin. In the special case where $\operatorname{deg} \phi \leq \operatorname{deg} x+\operatorname{deg} y+\operatorname{deg} z$, the equation $\phi=0$ defines a Poisson surface with either simple Kleinian, or elliptic singularity.

We study both commutative and noncommutative deformations of the corresponding Poisson algebra $\mathscr{B}_{\phi}$. We show that all Poisson algebra deformations are essentially obtained by deforming the polynomial $\phi$ (see Theorem 2.5.3). In the elliptic case, for instance, any such deformation gives the coordinate ring of an affine surface obtained by removing an elliptic curve from an appropriate projective del Pezzo surface.

Our approach to noncommutative deformations of elliptic singularities is motivated by the ideology explained in $\$ 1.2$. Specifically, we simultaneously deform both the corresponding surface $\phi=0$ and the ambient CY variety $\mathbb{C}^{3}$. This way, we construct a flat family of noncommutative CY algebras $\mathfrak{A}^{t}(\Phi)$ of dimension 3 , which provide a deformation of the Poisson algebra $\mathscr{A}_{\phi}$, and a family of central elements $\Psi \in \mathfrak{A}^{t}(\Phi)$. The noncommutative algebras of the form $\mathfrak{A}^{t}(\Phi) /\langle\langle\Psi\rangle$ thus provide a flat deformation of the Poisson algebra $\mathscr{B}_{\phi}$. In analogy with the Poisson case, these noncommutative algebras may be thought of as 'coordinate rings' of noncommutative del Pezzo surfaces.

There were a few other approaches to the problem of quantization of del Pezzo surfaces in the literature. One of them was proposed by M. Van den Bergh, in the paper [VB3], which gives a construction of the category of coherent sheaves on a 'would be' noncommutative (projective) del Pezzo surface. The connection between this approach and our approach is given by Chapter 12 of [VB3]. Namely, it is shown there that if one blows up six points in a quantum plane and then takes the affine part (the complement of the elliptic curve), then the coordinate ring is of the form $A /(n)$, where $A$ is a filtered deformation of an AS-regular algebra and $n$ is a normalizing element. We expect that this ring is the $E_{6}$-deformation considered in this paper, and that a similar approach works for $E_{7}$ and $E_{8}$.

A different construction, which is explicit but works only for a very special class of degenerate noncommutative del Pezzo surfaces, was proposed in [EOR].

Our present approach works in the general case, and is both quite simple and explicit. As a first step, we introduce a family of associative algebras $\mathfrak{A}^{t}(\Phi)$ to be the algebras with three generators, $x, y, z$, subject to three defining relations of the form

$$
[x, y]_{t}=\frac{\partial \Phi}{\partial z}, \quad[y, z]_{t}=\frac{\partial \Phi}{\partial x}, \quad[z, x]_{t}=\frac{\partial \Phi}{\partial y} .
$$

In this formula, $\Phi$ runs over a certain explicitly defined family of noncommutative cyclic potentials, $t$ is a complex parameter, and we have used the notation $[u, v]_{t}:=u v-t \cdot v u$. 
Remark 1.3.4. It is interesting to note that relations in 1.3 .3 look very similar to the formulas for the Poisson bracket (1.3.1), at least formally. The analogy goes much further since the actual formula for $\Phi$ (see (3.4.1)-3.4.2) is quite similar to the formula for the polynomial $\phi \in \mathbb{C}[x, y, z]$ that gives the equation of an affine del Pezzo surface (see (2.5.1-2.5.2).

Next, we prove one of our main results (see $\$ \$ 3.3-3.4$ ), saying that $\mathfrak{A}^{t}(\Phi)$ is a CalabiYau algebra of dimension 3 and that, for sufficiently general parameters, the center of $\mathfrak{A}^{t}(\Phi)$ has the form $\mathbb{C}[\Psi]$, a free polynomial algebra generated by an element $\Psi$ uniquely determined up to a constant summand. We show further that the family of noncommutative algebras of the form $\mathfrak{B}^{t}(\Phi, \Psi):=\mathfrak{A}^{t}(\Phi) /\langle\langle\Psi\rangle\rangle$ provides the required quantization of del Pezzo surfaces. It is also quite remarkable that, in a sense, any flat infinitesimal deformation of the Poisson algebra $\mathscr{B}_{\phi}$ can be obtained by the above construction (cf. Theorem 3.4.4).

In Section 3.5, we discuss the special case of homogeneous potentials. In this case, the algebras $\mathfrak{A}^{t}(\Phi)$ and $\mathfrak{B}^{t}(\Phi, \Psi)$ have natural gradings. The graded algebra $\mathfrak{A}^{t}(\Phi)$ is nothing but an Artin-Schelter regular algebra of dimension 3. These algebras, also known as Sklyanin algebras, have been intensively studied in the literature (see [AS], [ATV], $[\mathrm{AV}]$ and references therein). In particular, they were classified in D. Stephenson's Ph.D. thesis [St2] (see also [St3]). The best understood case is that of singularities of type $E_{6}$, resp. $E_{7}$, corresponding to quadratic, resp. cubic, Sklyanin algebras. The $E_{8}$-case has not been studied so well (cf. however [St1]).

The graded algebra $\mathfrak{B}^{t}(\Phi, \Psi)$ may be thought of as the homogeneous coordinate ring of a noncommutative elliptic singularity. There seems to be an interesting and largely unexplored theory of graded matrix factorizations for noncommutative elliptic singularities. In Section 3.6, we introduce a few basic results (cf. also [KST]) and formulate Conjecture 3.6 .8 .

In the general case of an arbitrary, not necessarily homogeneous, potential $\Phi$ the algebra $\mathfrak{A}^{t}(\Phi)$ comes equipped with a natural ascending filtration and one may form the corresponding Rees algebra. This way, one obtains a class of graded algebras that has been considered earlier, especially in type $E_{6}$ (see $[\overline{\mathrm{BSV}}]$ and $[\mathrm{C} 1]$, [C2]). Nonetheless, an explicit expression for the central element $\Psi \in \mathfrak{A}^{t}(\Phi)$, or the corresponding homogeneous central element of the Rees algebra, is quite complicated even in type $E_{6}$ (see $\$ 9$ and $[\bar{R}])$.

Remark 1.3.5. It would be interesting to establish a connection between our approach to noncommutative del Pezzo surfaces and the results of Chan-Kulkarni [CK].

\subsection{Definition of Calabi-Yau algebras}

We will work with unital associative, not necessarily commutative, $\mathbb{C}$-algebras, to be referred to as 'algebras'. We write $\otimes=\otimes_{\mathbb{C}}, \operatorname{dim}=\operatorname{dim}_{\mathbb{C}}$, etc.

Definition 1.4.1 ([Gi]). An algebra $A$ is said to be a Calabi-Yau algebra of dimension $d \geq 1$ provided it has finite Hochschild dimension, and there are $A$-bimodule isomor- 
phisms

$$
\operatorname{Ext}_{A \text {-bimod }}^{k}(A, A \otimes A) \cong \begin{cases}A & \text { if } k=d, \\ 0 & \text { if } k \neq d .\end{cases}
$$

The image of $1_{A} \in A$ under such an isomorphism gives a central element in $\operatorname{Ext}_{A \text {-bimod }}^{d}(A, A \otimes A)$, called the noncommutative volume on $A$.

Example 1.4.3. Let $X$ be a smooth connected affine complex algebraic variety of dimension $d$. A noncommutative volume for the algebra $A=\mathbb{C}[X]$, the coordintate ring of $X$, is the same thing as a nowhere vanishing section of the line bundle $\wedge^{d} T_{X}=$ $\mathscr{E} x t_{\mathcal{O}_{X \times X}}^{d}\left(\mathcal{O}_{X}, \mathcal{O}_{X \times X}\right)$. Thus, $A$ is a Calabi-Yau algebra if and only if $X$ is a CalabiYau variety.

Remark 1.4.4. Following Van den Bergh [VB1], it may be natural to consider a wider class of twisted $\mathrm{CY}$ algebras which satisfy a weaker version of (1.4.1) requiring that the group $\operatorname{Ext}_{A \text {-bimod }}^{k}(A, A \otimes A)$ be zero for $k \neq d$ and, for $k=d$, this Ext-group be an arbitrary invertible $A$-bimodule $U$, not necessarily $U=A$. Twisted $C Y$ algebras correspond geometrically to arbitrary Gorenstein varieties whose dualizing sheaf is a not necessarily trivial line bundle.

One should be able to develop an analogue of the theory of CY algebras in this more general framework. In such a theory, the role of $\mathrm{d} \Phi$, an exact noncommutative cyclic 1 -form associated with a cyclic potential $\Phi$ (cf. \$3.1 and [Gi, §3.5]) is expected to be played by a suitable noncommutative cyclic 1 -form with coefficients in $U^{-1}$, an inverse A-bimodule.

In the special case of graded algebras, any invertible graded $A$-bimodule $U$ must be a rank 1 free left $A$-module. The right $A$-action on $U$ is then given, in terms of a left $A$-module isomorphism $U \cong A$, by the formula $u a=u \cdot \sigma(a)$ for $u \in U, a \in A$, where $\sigma$ is an algebra automorphism of $A$. In the framework of Sklyanin algebras, this has the effect that the central element $\Psi$ of the CY algebra gets replaced by a normal element in a twisted CY algebra (cf. [ATV]).

\section{Poisson deformations of a quasi-homogeneous surface singularity}

\subsection{Deformations and cohomology}

Deformations of an algebraic object $A$ are often controlled by the vector space $H^{2}(A)$, the second cohomology group for an appropriate cohomology theory. That means, in particular, that associated with such a deformation, i.e. with a family of objects $\left\{A_{s} \mid\right.$ $s \in S\}$ parametrized by a scheme $S$, one has a canonical classifying, Kodaira-Spencer type, linear map

$$
\mathrm{KS}_{s}: T_{s} S \rightarrow H^{2}\left(A_{s}\right), \quad s \in S,
$$

where $T_{S} S$ stands for the Zariski tangent space to the scheme $S$ at a point $s$. A tangent vector $v \in T_{S} S$ determines a 1-parameter infinitesimal first order deformation of the object $A_{s}$. The image of $v$ under the classifying map $\mathrm{KS}_{s}$ is called the Kodaira-Spencer class of that deformation. 
Definition 2.1.2. A family $\left\{A_{s} \mid s \in S\right\}$, parametrized by a smooth scheme $S$, is said to be a (smooth) semiuniversa 1 deformation provided the classifying map is a vector space isomorphism for any $s \in S$.

Obstructions to deformations of an object $A$ are often controlled by $H^{3}(A)$, the third cohomology group. A standard result of deformation theory ensures the existence of a formal semiuniversal deformation of $A$ with base $S=H^{2}(A)$ provided one has: (1) $\operatorname{dim} H^{2}(A)<\infty$ and, moreover, (2) $H^{3}(A)=0$. However, a formal semiuniversal deformation of $A$ sometimes exists even if $H^{3}(A) \neq 0$. If the semiuniversal deformation exists, one says that the deformations of $A$ are unobstructed.

Given an associative, resp. commutative associative or Poisson, algebra $A$, one can define its Hochschild cohomology $H^{\bullet}(A):=\operatorname{Ext}_{A \text {-bimod }}(A, A)$ (Gerstenhaber), resp. Harrison cohomology, $\operatorname{Harr}^{\bullet}(A)$ (cf. [Lo] and references therein), or Poisson cohomology $P H^{\bullet}(A)$ (cf. [GK, Appendix] and $\$ 5.1$ below). By definition, in degree zero for an associative algebra $A$ one has $H H^{0}(A)=Z(A)$, the center of $A$. Similarly, for a Poisson algebra with Poisson bracket $\{-,-\}: A \times A \rightarrow A$, we have $P H^{0}(A)=\mathcal{Z}(A):=$ $\{z \in A \mid\{z, a\}=0, \forall a \in A\}$, is the Poisson center of $A$.

Also, for the corresponding degree zero Hochschild, resp. Poisson, homology, one has $H H_{0}(A)=A_{\text {cyc }}:=A /[A, A]$, the commutator quotient space, resp. $P H_{0}(A)=$ $A /\{A, A\}$.

Flat deformations of an associative, resp. commutative associative or Poisson, algebra $A$ are controlled by the second Hochschild cohomology group $H H^{2}(A)$, resp. $\operatorname{Harr}^{2}(A)$ or $\mathrm{PH}^{2}(A)$ (cf. [GK]). Thus, one may consider flat deformations of such an algebra $A$. Observe that a flat family of Poisson algebras is in particular a flat family of commutative algebras. This corresponds, in terms of cohomology, to the existence for any Poisson algebra $A$ of a canonical linear map can $: P H^{2}(A) \rightarrow \operatorname{Harr}^{2}(A)$.

Now, let $A$ be a Calabi-Yau algebra of dimension $d$ in the sense of Definition 1.4.1 According to [VB2], a choice of noncommutative volume for $A$ induces a Poincaré duality type isomorphism

$$
H H \cdot(A) \stackrel{\sim}{\rightarrow} H H^{d-\bullet}(A) .
$$

Following [CBEG], we introduce a BV operator $\Delta: H H^{\bullet}(A) \rightarrow H H^{\bullet-1}(A)$, obtained by transporting the Connes differential $B$, on Hochschild homology, to Hochschild cohomology via the duality isomorphism 2.1.3.

One may consider first order deformations of the CY algebra $A$ within the class of Calabi-Yau algebras. The Kodaira-Spencer classes of all such deformations form a vector subspace in $H H^{2}(A)$, which turns out to be equal to

$$
\operatorname{Ker}\left[\Delta: H H^{2}(A) \rightarrow H H^{1}(A)\right]
$$

\footnotetext{
1 The term "semiuniversal deformation" is often used for deformations parametrized by arbitrary (not necessarily smooth) formal schemes. In this paper, we will consider only smooth semiuniversal deformations, and for this reason will not explicitly mention that they are smooth.
} 
In the special case of Calabi-Yau algebras of dimension $d=3$, there is a chain of maps

$$
\kappa: A_{\mathrm{cyc}} \stackrel{2.1 .3}{\longrightarrow} H H^{3}(A) \stackrel{\Delta}{\longrightarrow} \operatorname{Ker}\left[\Delta: H H^{2}(A) \rightarrow H H^{1}(A)\right]
$$

where we have used that $\operatorname{Image}(\Delta) \subset \operatorname{Ker}(\Delta)$, since $\Delta^{2}=0$.

Let $A=\mathfrak{A}(\Phi)$ be a Calabi-Yau algebra of dimension 3 defined by a potential $\Phi$ (see $\$ 3.1$. An arbitrary infinitesimal variation $\Phi \rightsquigarrow \Phi+\varepsilon \Phi^{\prime}$ (where $\varepsilon^{2}=0$ ) of the potential yields an infinitesimal deformation of $A$. We show in $\$ 7.3$ below (cf. also [BT]) that such a deformation is automatically flat; moreover, it is a deformation within the class of Calabi-Yau algebras. Let $\Phi_{\text {cyc }}^{\prime} \in A_{\text {cyc }}$ denote the class of $\Phi^{\prime}$ in the commutator quotient. Then it is not difficult to prove the following proposition, whose proof is left to the reader.

Proposition 2.1.5. The Kodaira-Spencer class in $\operatorname{Ker}\left[\Delta: H H^{2}(A) \rightarrow H H^{1}(A)\right]$ of the deformation $\mathfrak{A}(\Phi) \rightsquigarrow \mathfrak{A}\left(\Phi+\varepsilon \Phi^{\prime}\right)$ is equal to $\kappa\left(\Phi_{\text {cyc }}^{\prime}\right)$, the image of $\Phi_{\text {cyc }}^{\prime}$ under the composite map 2.1.4.

\subsection{Quasi-homogeneous surface singularities.}

Let the multiplicative group $\mathbb{C}^{\times}$act on $\mathbb{C}^{3}$ with positive integral weights $a \leq b \leq c$. This makes the coordinate ring $\mathbb{C}[x, y, z]$ of $\mathbb{C}^{3}$, a nonnegatively graded algebra with homogeneous generators of degrees $\operatorname{deg} x=a, \operatorname{deg} y=b, \operatorname{deg} z=c$. Thus, $\phi \in \mathbb{C}[x, y, z]$ is a (weighted-, equivalently, quasi-) homogeneous polynomial of weight $\operatorname{deg} \phi=d$ if and only if one has $\mathrm{eu}(\phi)=d \cdot \phi$, where

$$
\mathrm{eu}:=a x \frac{\partial}{\partial x}+b y \frac{\partial}{\partial y}+c z \frac{\partial}{\partial z}
$$

denotes the Euler vector field that generates the $\mathbb{C}^{\times}$-action.

Associated with any polynomial $\phi \in \mathbb{C}[x, y, z]$ with an isolated singularity is its Jacobi ring $\mathbb{J}(\phi):=\mathbb{C}[x, y, z] /\left(\frac{\partial \phi}{\partial x}, \frac{\partial \phi}{\partial y}, \frac{\partial \phi}{\partial z}\right)$. If $\phi$ is (weighted-) homogeneous of weight $d$, then $0 \in \mathbb{C}^{3}$ is the only singular point. Furthermore, the Jacobi ring acquires a natural grading $\mathbb{J}(\phi)=\bigoplus_{m \geq 0} \mathbb{J}^{(m)}(\phi)$. For the corresponding Hilbert-Poincaré polynomial, one easily finds the formula (cf. $\$ 5.3$.

$$
\sum_{m \geq 0} u^{m} \cdot \operatorname{dim} \mathbb{J}^{(m)}(\phi)=\frac{\left(u^{d-a}-1\right)\left(u^{d-b}-1\right)\left(u^{d-c}-1\right)}{\left(u^{a}-1\right)\left(u^{b}-1\right)\left(u^{c}-1\right)} .
$$

Set $\mathcal{M}_{\phi}:=\phi^{-1}(0) \subset \mathbb{C}^{3}$. Specializing the RHS of 2.2.2) at $u \rightarrow 1$, we get a formula

$$
\operatorname{dim} \mathbb{J}(\phi)=\mu:=\frac{(d-a)(d-b)(d-c)}{a b c},
$$

for the Milnor number of the isolated singularity (at the origin) of the hypersurface $\mathcal{M}_{\phi}$.

Let $a \leq b \leq c<d$ be an arbitrary quadruple of positive integers such that $\operatorname{gcd}(a, b, c, d)=1$. According to Kyoji Saito [Sa Theorem 3], one has the following result. 
Theorem 2.2.4 (Saito). Assume that the rational function associated with the quadruple $(a, b, c ; d)$ by the formula on the right of $(2.2 .2)$ is a polynomial (i.e. has no poles). Then the surface $\mathcal{M}_{\phi}$ has an isolated singularity at the origin, for any general enough homogeneous polynomial $\phi \in \mathbb{C}[x, y, z]$ of degree $d$.

\subsection{Simple Kleinian and elliptic singularities}

Let $\mathbb{P}^{a, b, c}=\left(\mathbb{C}^{3} \backslash\{0\}\right) / \mathbb{C}^{\times}$denote the weighted projective plane corresponding to the $\mathbb{C}^{\times}$-action with weights $(a, b, c)$, where $\operatorname{gcd}(a, b, c)=1$. Restricting the projection $\mathbb{C}^{3} \backslash\{0\} \rightarrow \mathbb{P}^{a, b, c}$ to the punctured hypersurface, one obtains a map $\mathcal{M}_{\phi} \backslash\{0\} \rightarrow$ $\mathbb{P}\left(\mathcal{M}_{\phi}\right) \subset \mathbb{P}^{a, b, c}$. This way $\mathcal{M}_{\phi} \backslash\{0\}$ becomes a principal $\mathbb{C}^{\times}$-bundle over $\mathbb{P}\left(\mathcal{M}_{\phi}\right)$, a projective curve. The type of the hypersurface $\mathcal{M}_{\phi}$ is closely related to the integer

$$
\varpi:=d-a-b-c .
$$

There is a complete list of all hypersurfaces with $\varpi=-1,0,1$ (see [Sa] $)$. According to K. Saito, for any such hypersurface, one has $\mathcal{M}_{\phi} \backslash\{0\} \cong H_{\varpi} / \Gamma$. Here, $H_{\varpi}$ is the total space of the $\mathbb{C}^{\times}$-bundle associated with the canonical line bundle on a curve $C_{\varpi}$, and $\Gamma$ is a discrete group of bundle automorphisms. Depending on whether $\varpi=-1,0$, or +1 , the curve $C_{\varpi}$ is either the projective line $\mathbb{P}^{1}(\mathbb{C})$, the affine line $\mathbb{C}$, or the upper half-plane, respectively. Moreover, in each case, the group $\Gamma$ is a discrete subgroup of the group of motions of $C_{\varpi}$, viewed as a Riemann surface with the natural metric, and the $\Gamma$-action on $H_{\varpi}$ is induced by the natural $\Gamma$-action on $C_{\varpi}$.

In the case $\varpi=-1$, the surface $\mathcal{M}_{\phi}$ has a simple $A, D, E$ (Kleinian) singularity, while the case $\varpi=0$ corresponds to simple elliptic singularities $\widetilde{E}_{6}, \widetilde{E}_{7}, \widetilde{E}_{8}$ (for a reducible curve, all components must be rational). Specifically, one has the following classical result (cf. e.g. [B], and $\$ 6.1$ below).

Proposition 2.3.2. Let the variables $x, y, z$ have degrees $0<a \leq b \leq c$ such that $\operatorname{gcd}(a, b, c)=1$.

(i) Let $\phi \in \mathbb{C}[x, y, z]$ be an irreducible homogeneous polynomial of degree deg $\phi \leq$ $a+b+c$. Then the projective curve $\phi(x, y, z)=0$ is either rational or elliptic.

(ii) Let $d \leq a+b+c$ be such that, for a general homogeneous polynomial $\phi$ of degree $d$, the projective curve $\phi(x, y, z)=0$ is elliptic. Then $d=a+b+c$, and we have:

- One of the following holds:

\begin{tabular}{|ll|ccc|c|ccc|c|}
\hline & & $a$ & $b$ & $c$ & $d$ & $p:=d / a$ & $q:=d / b$ & $r:=d / c$ & $\mu$ \\
\hline$E_{6}$ & case & 1 & 1 & 1 & 3 & 3 & 3 & 3 & 8 \\
$E_{7}$ & case & 1 & 1 & 2 & 4 & 4 & 4 & 2 & 9 \\
$E_{8}$ & case & 1 & 2 & 3 & 6 & 6 & 3 & 2 & 10 \\
\hline
\end{tabular}

moreover, the integers $(p-1, q-1, r-1)$ give the lengths of three legs of the corresponding extended Dynkin diagram of type $\widetilde{E}_{6}, \widetilde{E}_{7}$, or $\widetilde{E}_{8}$. 
- The homogeneous equation of the corresponding elliptic curve can be brought to the canonical form

$$
\phi^{\tau}(x, y, z)=\frac{x^{p}}{p}+\frac{y^{q}}{q}+\frac{z^{r}}{r}+\tau \cdot x y z=0, \quad \text { where } \tau \in \mathbb{C}^{\times} .
$$

We note that in the setting of 2.3 .3 one has

$$
\frac{1}{p}+\frac{1}{q}+\frac{1}{r}=\frac{a}{d}+\frac{b}{d}+\frac{c}{d}=\frac{a+b+c}{d}=1 .
$$

Remark 2.3.5. The case $\varpi=1$ turns out to be closely related to 14 exceptional singularities (Dolgachev singularities) arising in degenerations of $K 3$ surfaces.

\section{4.}

Let $\mathbb{C}^{\times}$act on $\mathbb{C}^{3}$ with weights $0<a \leq b \leq c$, where $\operatorname{gcd}(a, b, c)=1$. Associated with $\phi \in \mathbb{C}[x, y, z]$ we have the Poisson algebra $\mathscr{B}_{\phi}$ (see Definition 1.3.2.

The following theorem will be proved in Subsection 5.5 using some results of Pichereau $([\overline{\mathrm{P}}]$, explained in $\$ 5.4$.

Theorem 2.4.1. For a (quasi-) homogeneous polynomial $\phi$ with an isolated singularity, we have:

(i) The Hochschild cohomology of $\mathscr{B}_{\phi}$ is

$$
H H^{\bullet}\left(\mathscr{B}_{\phi}\right) \cong \mathfrak{X}^{\bullet} \mathscr{B}_{\phi} \oplus u^{2} \cdot \mathbb{C}^{\bullet}[u] \otimes \mathbb{J}(\phi), \quad \operatorname{deg} u=1 .
$$

(ii) The Poisson cohomology of $\mathscr{B}_{\phi}$ is

$$
\begin{array}{ll}
P H^{0}\left(\mathscr{B}_{\phi}\right)=\mathbb{C}, & P H^{2}\left(\mathscr{B}_{\phi}\right)=\mathbb{J}^{(\varpi)}(\phi) \oplus \mathbb{J}(\phi), \\
P H^{1}\left(\mathscr{B}_{\phi}\right)=\mathbb{J}^{(\varpi)}(\phi), & P H^{k}\left(\mathscr{B}_{\phi}\right)=\mathbb{J}(\phi), \quad k \geq 3 .
\end{array}
$$

Here, in part (i), $\mathfrak{X}^{\bullet} \mathscr{B}_{\phi}$ denotes the algebra of polyderivations of the algebra $\mathscr{B}_{\phi}$ (cf. $\$ 4.1$, and in part (ii) we use the notation 2.3.1.

Theorem 2.4.1 ii) shows that the group $P H^{3}\left(\mathscr{B}_{\phi}\right)$ does not vanish. Nonetheless, there is an explicit Poisson deformation of the Poisson algebra $\mathscr{B}_{\phi}$ such that the tangent space to the base of that deformation is identified with $P H^{2}\left(\mathscr{B}_{\phi}\right)=\mathbb{J}^{(\varpi)}(\phi) \oplus \mathbb{J}(\phi)$. Specifically, the space $\mathbb{J}(\phi)$, the second direct summand, parametrizes deformations of the Poisson algebra $\mathscr{B}_{\phi}$ obtained by deformations of the polynomial $\phi$. Any nontrivial deformation of this kind gives a nontrivial deformation of $\mathscr{B}_{\phi}$, viewed as a commutative algebra (with the Poisson structure disregarded; cf. relation to Harrison cohomology below).

On the other hand, the space $\mathbb{J}^{(\varpi)}(\phi)$, the first direct summand in the decomposition $P H^{2}\left(\mathscr{B}_{\phi}\right)=\mathbb{J}^{(\varpi)}(\phi) \oplus \mathbb{J}(\phi)$, parametrizes deformations which change the Poisson structure on $\mathscr{B}_{\phi}$ while keeping the commutative algebra structure unaffected. To see this, we use results of Pichereau $[\mathrm{P}]$ (see also formula $[5.5 .1)$ ). According to $[\overline{\mathrm{P}}]$, elements of the direct summand $\mathbb{J}^{(\varpi)}(\phi) \subset P H^{2}\left(\mathscr{B}_{\phi}\right)$ may be represented by bivectors of the form $f \cdot \pi$, 
where $f \in \mathscr{B}_{\phi}$ is a homogeneous element of degree $\varpi$, and $\pi$ is the Poisson bivector that gives the Poisson bracket (1.3.1) on $\mathscr{B}_{\phi}$. We will see in the course of the proof of Theorem 2.4.1 that the family of bivectors of the form $\pi+f \cdot \pi, f \in \mathbb{J}^{(\varpi)}(\phi)$, yields the required family of nontrivial deformations of the Poisson structure on $\mathscr{B}_{\phi}$, parametrized by the vector space $\mathbb{J}^{(\varpi)}(\phi)$.

The direct sum decomposition $H H^{2}\left(\mathscr{B}_{\phi}\right)=\mathfrak{X}^{2} \mathscr{B}_{\phi} \oplus u \cdot \mathbb{J}(\phi)$, in Theorem 2.4.1 (i), corresponds to the Hodge decomposition of Hochschild cohomology (cf. [Lo, §4.5]). The second direct summand is equal to $\operatorname{Harr}^{2}\left(\mathscr{B}_{\phi}\right)$, the second Harrison cohomology group of the algebra $\mathscr{B}_{\phi}$. By general deformation theory, the latter group is the base of the semiuniversal unfolding of the quasi-homogeneous isolated singularity $\phi=0$. Thus, the canonical morphism can : $\operatorname{PH}^{2}\left(\mathscr{B}_{\phi}\right) \rightarrow \operatorname{Harr}^{2}\left(\mathscr{B}_{\phi}\right)$, which sends a Poisson deformation to the corresponding deformation of the underlying commutative algebra, may be identified with the second projection $\mathbb{J}^{(\varpi)}(\phi) \oplus \mathbb{J}(\phi) \rightarrow \mathbb{J}(\phi)$. This agrees with the discussion of the preceding paragraph: the direct summand which corresponds to Poisson deformations of $\mathscr{B}_{\phi}$ induced by deformations of the polynomial $\phi$ projects isomorphically onto the group $\operatorname{Harr}^{2}\left(\mathscr{B}_{\phi}\right)$. On the other hand, the direct summand $\mathbb{J}^{(\varpi)}(\phi)$, which corresponds to deformations of the Poisson structure which do not change the commutative algebra structure, projects to zero.

Note that if $\varpi=-1$, the case of Kleinian singularity, Theorem 2.4.1 yields $P H^{1}\left(\mathscr{B}_{\phi}\right)$ $=0$ and $P H^{2}\left(\mathscr{B}_{\phi}\right)=\operatorname{Harr}^{2}\left(\mathscr{B}_{\phi}\right)=\mathbb{J}(\phi)$. It is easy to see that, in this case, the map can reduces to the identity.

\subsection{Poisson deformations of elliptic singularities}

Given a triple $(p, q, r)$ of positive integers, introduce a triple of polynomials

$$
\begin{aligned}
& P=\frac{1}{p} \cdot x^{p}+\alpha_{1} \cdot x^{p-1}+\cdots+\alpha_{p-1} \cdot x \in \mathbb{C}[x], \\
& Q=\frac{1}{q} \cdot y^{q}+\beta_{1} \cdot y^{q-1}+\cdots+\beta_{q-1} \cdot y \in \mathbb{C}[y], \\
& R=\frac{1}{r} \cdot z^{r}+\gamma_{1} \cdot z^{r-1}+\cdots+\gamma_{r-1} \cdot z \in \mathbb{C}[z] .
\end{aligned}
$$

Further, we let

$$
\phi_{P, Q, R}^{\tau, v}:=\tau \cdot x y z+P(x)+Q(y)+R(z)+v \in \mathbb{C}[x, y, z], \quad \tau \in \mathbb{C}^{\times}, v \in \mathbb{C} .
$$

The family of polynomials $\phi_{P, Q, R}^{\tau, v}$ depends on $(p-1)+(q-1)+(r-1)+2=p+q+r-1$ complex parameters $\alpha_{i}, \beta_{j}, \gamma_{k}, \tau$, $v$. If all the parameters, except $\tau$, vanish, this family specializes to a homogeneous polynomial $\phi^{\tau}=\phi_{0,0,0}^{\tau, 0}$ of the form 2.3.4.

Recall that, for any polynomial $\phi \in \mathbb{C}[x, y, z]$, the equation $\phi(x, y, z)=0$ defines an affine Poisson surface in $\mathbb{C}^{3}$, with coordinate ring $\mathscr{B}_{\phi}$.

Theorem 2.5.3. Let $(a, b, c)$ and $(p, q, r)$ be the integers associated to one of the three cases $E_{\ell}, \ell=6,7,8$, of table (2.3.3), and let $\phi^{\tau}$ be the corresponding polynomial 2.3.4). Then 
(i) For the Milnor number $\operatorname{dim} \rrbracket\left(\phi^{\tau}\right)=\mu$, we have

$$
\mu=\frac{(a+b)(a+c)(b+c)}{a b c}=p+q+r-1 .
$$

(ii) The equations $\phi_{P, Q, R}^{\tau, v}(x, y, z)=0$ give a flat $\mu$-parameter family of affine del Pezzo surfaces of the corresponding type $E_{\ell}, \ell=6,7,8$.

(iii) The family of Poisson algebras $\left\{\mathscr{B}_{\phi} \mid \phi=c \cdot \phi_{P, Q, R}^{\tau, \nu}, c \in \mathbb{C}^{\times}\right\}$provides a semiuniversal Poisson deformation of $\mathscr{B}_{\phi^{\tau}}$, the coordinate ring of the corresponding elliptic singularity 2.3.4).

In the next section, we will state a 'quantum analogue' of Theorem 2.5.3 with Poisson algebras being replaced by noncommutative algebras.

Remark 2.5.5. Observe that the family of Poisson algebras $\mathscr{B}_{\phi}$, in part (iii), depends on $\mu+1$ parameters. The reason is that, although the underlying surface $\phi^{\tau}=0$ does not depend on the extra parameter $c \in \mathbb{C}^{\times}$, the corresponding Poisson structure does.

Proof of Theorem 2.5.3. Part (i) is a simple consequence of the equations $d=a+b+c$ and $p=d / a, q=d / b, r=d / c$, combined with formula 2.2.3. Part (ii) is a well known classical result (cf. $[\mathrm{D}])$.

Next, let $S=\mathbb{C}^{2} \times S_{p} \times S_{q} \times S_{r} \times \mathbb{C}^{\times}$. Here, the parameters $\tau, v$ form coordinates in the first factor $\mathbb{C}^{2}$, the affine linear spaces $S_{p}, S_{q}, S_{r}$ are spanned by the corresponding polynomials in 2.5.1, and the parameter $c$ gives a coordinate on the last factor $\mathbb{C}^{\times}$. Thus, members of the family $\left\{\mathscr{B}_{\phi} \mid \phi=c \cdot \phi_{P, Q, R}^{\tau, \nu}, c \in \mathbb{C}^{\times}\right\}$are parametrized by points of $S$. Let $o \in S$ be the point corresponding to vanishing parameters $v, c, P, Q, R$, i.e., to the Poisson algebra $\mathscr{B}_{\phi^{\tau}}$.

To prove (iii), we must show that the classifying map for our family of Poisson algebras induces a vector space isomorphism $T_{o} S \stackrel{\sim}{\rightarrow} P H^{2}\left(\mathscr{B}_{\phi^{\tau}}\right)$. According to Theorem 2.4.1 (cf. also the discussion at the beginning of this subsection), we have $P H^{2}\left(\mathscr{B}_{\phi^{\tau}}\right)=$ $\mathbb{J}\left(\phi^{\tau}\right) \oplus \mathbb{C}$, where the direct summand $\mathbb{C}$ corresponds to the 1-dimensional space $\mathbb{J}^{(\varpi)}\left(\phi^{\tau}\right)$. By part (i), we compute

$\operatorname{dim} P H^{2}\left(\mathscr{B}_{\phi^{\tau}}\right)=\operatorname{dim} \mathbb{J}\left(\phi^{\tau}\right)+1=\mu+1=(p-1)+(q-1)+(r-1)+3=\operatorname{dim} S$.

It is easy to see that the map $T_{o} S \rightarrow \mathbb{J}\left(\phi^{\tau}\right) \oplus \mathbb{C}$ we are interested in is the natural map sending a polynomial $c \cdot \phi_{P, Q, R}^{\tau, \nu}$ to its residue class in the Jacobi ring. This map is injective. Hence, it must be an isomorphism, due to the above equality of dimensions.

\section{Main results}

\subsection{Algebras defined by a potential}

Let $V$ be a $\mathbb{C}$-vector space with basis $x_{1}, \ldots, x_{n}$, and let $F=T V=\mathbb{C}\left\langle x_{1}, \ldots, x_{n}\right\rangle$ be the corresponding free tensor algebra. The commutator quotient space $F_{\text {cyc }}=F /[F, F]$ is a $\mathbb{C}$-vector space with the natural basis formed by cyclic words in the alphabet $x_{1}, \ldots, x_{n}$. Elements of $F_{\text {cyc }}$ are referred to as potentials. 
Let $\Phi \in F_{\text {cyc }}$. For each $j=1, \ldots, n$, one defines $\partial_{j} \Phi \in F$, the corresponding partial derivative of the potential, by the formula

$$
\partial_{j} \Phi:=\sum_{\left\{s \mid i_{s}=j\right\}} x_{i_{s}+1} x_{i_{s}+2} \ldots x_{i_{r}} x_{i_{1}} x_{i_{2}} \ldots x_{i_{s}-1} \in \mathbb{C}\left\langle x_{1}, \ldots, x_{n}\right\rangle .
$$

We extend this definition to arbitrary elements $\xi=\left(\xi_{1}, \ldots, \xi_{n}\right) \in \mathbb{C}^{n}$ by $\mathbb{C}$-linearity, i.e. we put $\partial_{\xi} \Phi:=\xi_{1} \cdot \partial_{1} \Phi+\cdots+\xi_{n} \cdot \partial_{n} \Phi$. This way, we get a linear map $V^{*} \rightarrow T V$, $\xi \mapsto \partial_{\xi} \Phi$.

Many interesting examples of Calabi-Yau algebras arise from the following construction of algebras associated with a potential (cf. [Gi]). Given $\Phi \in F_{\text {cyc }}$, introduce an associative algebra

$$
\mathfrak{A}(\Phi):=F /\langle\partial \partial \Phi\rangle=\mathbb{C}\left\langle x_{1}, \ldots, x_{n}\right\rangle /\left\langle\left\langle\partial_{i} \Phi\right\rangle_{i=1, \ldots, n},\right.
$$

a quotient of $F$ by the two-sided ideal generated by all $n$ partial derivatives $\partial_{i} \Phi, i=$ $1, \ldots, n$, of the potential $\Phi$.

\subsection{Filtered setting}

Let each of the generators $x_{k}, k=1, \ldots, n$, be assigned some positive degree deg $x_{k}=$ $d_{k} \geq 1$. This makes $V$ a graded vector space, with homogeneous basis $x_{k}, k=1, \ldots, n$. Thus, the tensor algebra $F=T V=\mathbb{C}\left\langle x_{1}, \ldots, x_{n}\right\rangle$ acquires a graded algebra structure with respect to the induced total grading $F=\bigoplus_{r \geq 0} F^{(r)}$ (not to be confused with the standard grading on the tensor algebra; the latter corresponds to the special case where $\operatorname{deg} x_{k}=1$ for all $k$ ).

One may also view $F$ as a filtered algebra, with an increasing filtration $\mathbb{C}=F^{\leq 0} \subset$ $F^{\leq 1} \subset \cdots$ given by $F^{\leq r}=F^{(0)} \oplus \cdots \oplus F^{(r)}$. The filtration, resp. grading, on $F$ gives rise to a filtration $F_{\mathrm{cyc}}^{\leq k}, k=0,1, \ldots$, resp. grading $F_{\mathrm{cyc}}=\bigoplus_{r} F_{\mathrm{cyc}}^{(r)}$, on the commutator quotient space $F_{\text {cyc }}$.

The increasing filtration on $F$ induces a filtration $\mathbb{C}=\mathfrak{A}^{\leq 0}(\Phi) \subset \mathfrak{A}^{\leq 1}(\Phi) \subset$ $\mathfrak{A}^{\leq 2}(\Phi) \subset \cdots$ on the quotient algebra $\mathfrak{A}(\Phi)$. In the special case where $\Phi$ is, in effect, homogeneous, our algebra inherits a grading $\mathfrak{A}(\Phi)=\bigoplus_{m \geq 0} \mathfrak{A}^{(m)}(\Phi)$.

Given a filtered algebra $A$ with filtration by finite-dimensional vector spaces, we write

$$
\mathrm{P}(A):=\sum_{m \in \mathbb{Z}} \operatorname{dim}\left(\operatorname{gr}^{(m)} A\right) \cdot u^{m} \in \mathbb{Z}[[u]]
$$

for the Hilbert-Poincaré series of the associated graded algebra gr $A=\bigoplus_{m \geq 0} \operatorname{gr}^{(m)} A$.

An ascending filtration, resp. grading, on $A$ induces a filtration $H H_{\leq m}^{\circ}(A)$, resp. grading $H H^{\bullet}(A)=\bigoplus_{m \in \mathbb{Z}} H H_{(m)}^{\cdot}(A)$, on each Hochschild cohomology group.

A family of nonnegatively filtered algebras is said to be a semiuniversal filtered family provided the associated graded algebras form a flat family and, moreover, the classifying map gives an isomorphism $T_{S} S \stackrel{\sim}{\rightarrow} H H_{\leq 0}^{2}\left(A_{s}\right)$ for all $s \in S$. There is a similar definition in the case of graded algebras.

The above discussion also applies to filtered, resp. graded, Poisson algebras and Poisson cohomology. 


\subsection{Quantization of the Poisson algebras $\mathscr{A}_{\phi}$ and $\mathscr{B}_{\phi}$}

In the three sections below, we are going to state four theorems which are the main results of this paper. The proofs of these theorems will be given later, mostly in $\$ \$ 7,8$.

Fix a triple of integers $0<a \leq b \leq c$ such that $\operatorname{gcd}(a, b, c)=1$. We will be interested in (not necessarily commutative) algebras with three generators. We put $F=\mathbb{C}\langle x, y, z\rangle$ and view $F$ as a graded algebra such that $\operatorname{deg} x=a, \operatorname{deg} y=b, \operatorname{deg} z=c$.

It will be convenient to introduce the following

Definition 3.3.1. An element $\Phi \in F_{\text {cyc }}$ is called a CY-potential provided $\mathfrak{A}(\Phi)$ is a Calabi-Yau algebra of dimension 3.

The basic example of a homogeneous CY-potential of degree $d=a+b+c$ is $\Phi=$

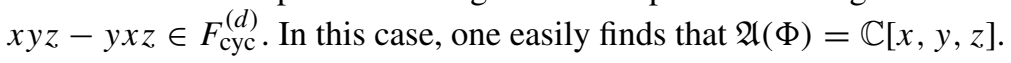

We will be mostly interested in general, not necessarily homogeneous, potentials of degree $d=a+b+c$.

Theorem 3.3.2. Let $(a, b, c)$ be a triple of positive integers and $\Phi^{(d)}$ a homogeneous $C Y$-potential of degree $d=a+b+c$. Then, for any potential $\Phi^{\prime} \in F_{\mathrm{cyc}}^{<d}$, one has:

(i) The sum $\Phi=\Phi^{(d)}+\Phi^{\prime}$ is a CY-potential, and the Hilbert-Poincaré series of the corresponding filtered algebra,

$$
\mathrm{P}(\mathfrak{A}(\Phi))=\frac{1}{\left(1-u^{a}\right)\left(1-u^{b}\right)\left(1-u^{c}\right)},
$$

coincides with the Hilbert-Poincaré series of the graded algebra $\mathbb{C}[x, y, z]$.

(ii) There exists a nonscalar central element $\Psi \in \mathfrak{A}^{\leq d}(\Phi)$.

Theorem 3.3.2 is proved in Subsection 8.3 .

The equation in part (i) of the theorem shows that any algebra of the form $\mathfrak{A}(\Phi)$, where $\Phi$ is a nonhomogeneous potential whose leading term is a CY-potential of degree $a+b+c$, may be thought of as a 'noncommutative analogue' of the polynomial algebra $\mathbb{C}[x, y, z]$. Further, a Calabi-Yau structure (i.e. a noncommutative volume) on the algebra may be thought of as a noncommutative deformation of a unimodular Poisson structure on the polynomial algebra. As we will see in $\$ 4$ below, any such unimodular Poisson algebra must be of the form $\mathscr{A}_{\phi}$ for an appropriate polynomial $\phi \in \mathbb{C}[x, y, z]$. Moreover, the polynomial $\phi$ is necessarily a central element for the Poisson structure.

This suggests viewing a central element $\Psi \in \mathfrak{A}(\Phi)$ as a noncommutative analogue of the polynomial $\phi$. Thus, one may view any algebra of the form

$$
\mathfrak{B}(\Phi, \Psi):=\mathfrak{A}(\Phi) /\langle\Psi\rangle, \quad \Psi \in Z(\mathfrak{A}(\Phi)),
$$

(a quotient of the CY algebra $\mathfrak{A}(\Phi)$ by the two-sided ideal generated by the central element $\Psi$ ) as a noncommutative analogue of a Poisson algebra of the form $\mathscr{B}_{\phi}=\mathscr{A}_{\phi} /(\phi)$. 


\subsection{Noncommutative del Pezzo surfaces}

For the rest of Section 3 we assume that $(a, b, c)$ is one of the triples from table 2.3.3 and recall the nonhomogeneous polynomials $\phi_{P, Q, R}^{\tau, \nu}$ of degree $d=a+b+c$ defined in 2.5.2. According to Theorem 2.5.3 (ii), the algebra $\mathscr{B}_{\phi}, \phi=\phi_{P, Q, R}^{\tau, v}$, gives the coordinate ring of an affine del Pezzo surface.

On the other hand, Theorem 3.3.2(ii) ensures the existence of nontrivial central elements in the noncommutative algebra $\mathfrak{A}(\Phi)$. Therefore, it is natural to look for cyclic potentials $\Phi$ of the form similar to one given by formula (2.5.2), and to view the corresponding algebras $\mathfrak{B}(\Phi, \Psi)$, in 3.3 .3 , as quantizations of those del Pezzo surfaces.

To implement this program, fix complex parameters $t, c$. To each triple $P \in \mathbb{C}[x]$, $Q \in \mathbb{C}[y], R \in \mathbb{C}[z]$, of polynomials given by formulas 2.5.1), of degrees $p, q, r$, respectively, we associate the potential

$$
\Phi_{P, Q, R}^{t, c}=x y z-t \cdot y x z+c \cdot[P(x)+Q(y)+R(z)] \in F_{\text {cyc }} .
$$

Clearly, $\Phi_{P, Q, R}^{t, c}$ is a nonhomogeneous potential of degree $d$. The corresponding algebra $\mathfrak{A}\left(\Phi_{P, Q, R}^{t, c}\right)$ is a filtered algebra with generators $x, y, z$, and the following three relations:

$$
x y-t \cdot y x=c \cdot \frac{d R(z)}{d z}, \quad y z-t \cdot z y=c \cdot \frac{d P(x)}{d x}, \quad z x-t \cdot x z=c \cdot \frac{d Q(y)}{d y} .
$$

We need the following

Definition 3.4.3. Let $X$ be an irreducible variety, thought of as a variety of 'parameters'. We say that a property $(\mathrm{P})$ holds for generic parameters $x \in X$ if there exists a countable family, $\left\{Y_{s}\right\}$, of closed subvarieties $Y_{s} \subsetneq X$ such that (P) holds for any $x \in X \backslash \bigcup_{s} Y_{s}$.

Recall formula 2.5.4) for the Milnor number $\mu=\operatorname{dim} \rrbracket(\phi)$ of an elliptic singularity. The two theorems below are our main results about noncommutative del Pezzo surfaces.

Theorem 3.4.4. For generic parameters $(t, c, P, Q, R)$, formula 3.4.1 gives a CY-potential, and we have:

(i) The algebras $\mathfrak{A}\left(\Phi_{P, Q, R}^{t, c}\right)$, with relations 3.4.2, form a semiuniversal filtered family of associative algebras that depends on $\mu$ parameters.

(ii) The algebras of the form $\mathfrak{B}\left(\Phi_{P, Q, R}^{t, c}, \Psi\right)$, where $\Psi \in \mathfrak{A}^{\leq d}\left(\Phi_{P, Q, R}^{t, c}\right)$ is a nonscalar central element, give a semiuniversal family of associative algebras that depends on $\mu+1$ parameters.

A sketch of proof of Theorem 3.4.4 is given in Subsection 8.5.

Our presentation for the algebras $\mathfrak{B}(\Phi, \Psi)$ in terms of generators and relations is not completely explicit yet, since we have not explicitly described the central elements $\Psi$. This can be done by a direct computation which has been carried out by Eric Rains (see $\$ 9$ and $[\mathrm{R}])$.

Part (1) of the next theorem gives a 'parametrization' of noncommutative del Pezzo algebras similar to the one provided, in the commutative (Poisson) case, by Theorem 2.5.3 (iii). 
Theorem 3.4.5. For any generic homogeneous potential $\Phi^{(d)}$ of degree $d=a+b+c$ and an arbitrary potential $\Phi^{\prime} \in F_{\mathrm{cyc}}^{<d}$, the sum $\Phi=\Phi^{(d)}+\Phi^{\prime}$ is a CY-potential, and the following holds:

(1) There exists a potential of the form $\Phi_{P, Q, R}^{t, c}$ (cf. 3.4.1) such that one has a filtered algebra isomorphism $\mathfrak{A}(\Phi) \cong \mathfrak{A}\left(\Phi_{P, Q, R}^{t, c}\right)$.

(2) The center of $\mathfrak{A}(\Phi)$ is a free polynomial algebra $\mathbb{C}[\Psi]$ generated by an element $\Psi \in$ $\mathfrak{A}^{\leq d}(\Phi)$, and one has $\operatorname{gr} Z(\mathfrak{A}(\Phi)) \cong Z\left(\mathfrak{A}\left(\Phi^{(d)}\right)\right)$.

Theorem 3.4 .5 is proved in Subsection 8.3

\subsection{Noncommutative elliptic singularities}

Let $(a, b, c)$ be one of the triples from table 2.3.3. In this subsection, we are interested in the special case where the polynomials $P, Q, R$ (cf. (2.5.1)) reduce to their leading terms. In that case, the corresponding potential $\Phi^{t, c}:=\Phi_{P, Q, R}^{t, c}$, and the central element $\Psi \in \mathfrak{A}^{\leq d}\left(\Phi^{t, c}\right)$, both become homogeneous elements of degree $\operatorname{deg} \Phi^{t, c}=\operatorname{deg} \Psi=$ $a+b+c=d$.

Explicitly, we have (cf. also $\$ 9$ and $[\mathbb{R}]$ )

\begin{tabular}{|c|c|c|}
\hline Case & $\Phi^{t, c} \in F_{\mathrm{cyc}}^{(d)}$ & $\Psi \in Z\left(\mathfrak{A}^{(d)}\left(\Phi^{t, c}\right)\right)$ \\
\hline$E_{6}$ & $x y z-t \cdot y x z+c\left(\frac{x^{3}}{3}+\frac{y^{3}}{3}+\frac{z^{3}}{3}\right)$ & $c \cdot y^{3}+\frac{t^{3}-c^{3}}{c^{3}+1}\left(y z x+c \cdot z^{3}\right)-t \cdot z y x$ \\
\hline$E_{7}$ & $x y z-t \cdot y x z+c\left(\frac{x^{4}}{4}+\frac{y^{4}}{4}+\frac{z^{2}}{2}\right)$ & $\left(t^{2}+1\right) x y x y-\frac{t^{4}+t^{2}+1}{t^{2}-c^{4}}\left(t \cdot x y^{2} x+c^{2} \cdot y^{4}\right)+t \cdot y^{2} x^{2}$ \\
\hline$E_{8}$ & $x y z-t \cdot y x z+c\left(\frac{x^{6}}{6}+\frac{y^{3}}{3}+\frac{z^{2}}{2}\right)$ & too long \\
\hline
\end{tabular}

Let $\chi(u)$ denote the rational function on the RHS of formula 2.2.2). Further, let $\Upsilon \in H H^{3}(\mathfrak{A}(\Phi))$ denote the image of $1 \in H H_{0}(\mathfrak{A}(\Phi))$ under the isomorphism in 2.1.3), resp. $\Delta$ denote the $\mathrm{BV}$-operator, associated with a noncommutative volume on the $\mathrm{CY}$ algebra $\mathfrak{A}(\Phi)$ (cf. Definition 1.4.1).

Theorem 3.5.2. Let $(a, b, c)$ be as in table 3.5.1. Then, for any generic homogeneous potential $\Phi$ of degree $d=a+b+c$, one has:

(i) There exists a potential of the form $\Phi^{t, c}$, as in table 3.5.1, such that one has a graded algebra isomorphism $\mathfrak{A}(\Phi) \cong \mathfrak{A}\left(\Phi^{t, c}\right)$.

(ii) Each group $H H^{k}(\mathfrak{A}(\Phi)), k \leq 3$, is a free $\mathbb{C}[\Psi]$-module with the Hilbert-Poincaré series

$$
\mathrm{P}\left(H H^{k}(\mathfrak{A}(\Phi))\right)= \begin{cases}\frac{1}{1-u^{d}} & \text { if } k=0,1, \\ \frac{1}{u^{d}\left[\frac{\chi(u)}{1-u^{d}}-1\right]} & \text { if } k=2, \\ \frac{\chi(u)}{u^{d}\left(1-u^{d}\right)} & \text { if } k=3 .\end{cases}
$$


(iii) The BV-operator kills $\Upsilon$ and induces the bijections

$$
\Delta: H H^{3}(\mathfrak{A}(\Phi)) / \mathbb{C} \cdot \Upsilon \stackrel{\sim}{\rightarrow} H H^{2}(\mathfrak{A}(\Phi)), \quad \text { resp. } \quad \Delta: H H^{1}(\mathfrak{A}(\Phi)) \stackrel{\sim}{\rightarrow} H H^{0}(\mathfrak{A}(\Phi)) .
$$

Theorem 3.5.2 is proved in Subsection 8.4

Remarks 3.5.3. (1) Part (ii) of the theorem is a generalization of a result of Van den Bergh [VB2]. The factor $u^{d}$ in denominators of the formulas is due to the fact that $\operatorname{deg} \Upsilon=-d$. We also recall that any Calabi-Yau algebra of dimension 3 has no Hochschild cohomology in degrees $>3$.

(2) For a result related to part (i) see also [BT, Proposition 5.4].

Associated with a nonzero homogeneous central element $\Psi \in \mathfrak{A}(\Phi)$, of degree $d$, there is the corresponding quotient algebra $\mathfrak{B}(\Phi, \Psi)$ (cf. (3.3.3)), which inherits a graded algebra structure. According to [ATV] and [St1], the element $\Psi$ is not a zero divisor in $\mathfrak{A}(\Phi)$; furthermore, the algebra $\mathfrak{B}(\Phi, \Psi)$ is a noetherian domain of Gelfand-Kirillov dimension two.

Let $D^{b}(\mathfrak{B}(\Phi, \Psi))$ be the bounded derived category of finitely generated graded left $\mathfrak{B}(\Phi, \Psi)$-modules. One also introduces Tails $(\mathfrak{B}(\Phi, \Psi)) \subset D^{b}(\mathfrak{B}(\Phi, \Psi))$, a full triangulated subcategory of tails, whose objects are complexes with finite-dimensional cohomology (cf. [NVB] $)$.

Recall next that, for any algebra of the form $\mathfrak{B}(\Phi, \Psi)$ as above, there exists a triple $(\mathbb{E}, \mathcal{L}, \sigma)$, where $\mathbb{E}$ is an elliptic curve, $\mathcal{L}$ is a positive line bundle on $\mathbb{E}$, and $\sigma$ is an automorphism of $\mathbb{E}$, such that one has a graded algebra isomorphism (see [ATV], [St1])

$$
\mathfrak{B}(\Phi, \Psi)=\bigoplus_{m \geq 0} \Gamma\left(\mathbb{E}, \mathcal{L} \otimes \sigma^{*} \mathcal{L} \otimes \cdots \otimes\left(\sigma^{m-1}\right)^{*} \mathcal{L}\right) .
$$

The graded algebra on the right of 3.5.4 is a $\sigma$-twisted homogeneous coordinate ring of $\mathbb{E}$. Therefore, the algebra $\mathfrak{B}(\Phi, \Psi)$ may be thought of as a flat graded noncommutative deformation of the affine cone over the elliptic curve $\mathbb{E}$.

Let $D^{b} \operatorname{Coh}(\mathbb{E})$ be the bounded derived category of coherent sheaves on $\mathbb{E}$. According to a result due to Artin and Van den Bergh [AV], there is a triangulated equivalence

$$
D^{b} \operatorname{Coh}(\mathbb{E}) \cong D^{b}(\mathfrak{B}(\Phi, \Psi)) / \operatorname{Tails}(\mathfrak{B}(\Phi, \Psi)) .
$$

\subsection{Matrix factorizations on a noncommutative singularity}

Given a nonnegatively graded algebra $A$ and a central homogeneous element $\Psi \in A$, of degree $d>0$, one may introduce $D_{\mathrm{gr}}(A, \Psi)$, a triangulated category of graded matrix factorizations (see [Or1]). An object of $D_{\mathrm{gr}}(A, \Psi)$ is a diagram

$$
M=\left(M_{+} \underset{g^{\prime}}{\stackrel{g}{\rightleftarrows}} M_{-}\right), \quad g \circ g^{\prime}=\Psi \cdot \operatorname{Id}_{M_{-}}, \quad g^{\prime} \circ g=\Psi \cdot \operatorname{Id}_{M_{+}},
$$


where $M_{+}, M_{-}$is a pair of finite rank free graded $A$-modules and $g, g^{\prime}$ is a pair of graded $A$-module morphisms of degrees 0 and $d$, respectively.

We take $A=\mathfrak{A}(\Phi)$ and apply a noncommutative version of results due to Orlov [Or1], [Or2]. This way, one obtains the following (cf. also [KST]).

Theorem 3.6.2. (i) There is a triangulated equivalence

$$
D^{b} \operatorname{Coh}(\mathbb{E}) \cong D_{\operatorname{gr}}(\mathfrak{A}(\Phi), \Psi) .
$$

(ii) Any maximal Cohen-Macaulay graded $\mathfrak{B}(\Phi, \Psi)$-module has a 2-periodic free graded $\mathfrak{A}(\Phi)$-module resolution.

Sketch of proof of Theorem 3.6.2 It is known that $\mathfrak{A}(\Phi)$, being a graded Calabi-Yau algebra, is automatically a Gorenstein, Artin-Schelter regular algebra of dimension 3 (see [BT], [ATV]). Further, the central element $\Psi$ is not a zero divisor in $\mathfrak{A}(\Phi)$, by construction. It follows that the quotient $\mathfrak{B}(\Phi, \Psi)=\mathfrak{A}(\Phi) /\langle\langle\Psi\rangle$ is an Auslander-Gorenstein algebra of dimension 2, by [Le].

Let $\operatorname{Perf}(\mathfrak{B}(\Phi, \Psi))$ denote the full triangulated subcategory in $D^{b}(\mathfrak{B}(\Phi, \Psi))$ of perfect complexes, i.e. of bounded complexes of free graded left $\mathfrak{B}(\Phi, \Psi)$-modules of finite rank. Following Orlov [Or1], one introduces a quotient category $D_{\mathrm{gr}}^{\text {sing }}(\mathfrak{B}(\Phi, \Psi)):=$ $D^{b}(\mathfrak{B}(\Phi, \Psi) / \operatorname{Perf}(\mathfrak{B}(\Phi, \Psi))$, the triangulated category of a homogeneous singularity.

An immediate generalization of [Or1, Theorem 3.9] yields the following result:

Proposition 3.6.3. Let $A=\bigoplus_{j>0} A_{j}$ be a graded noetherian algebra with $A_{0}=\mathbb{C}$. Assume that $A$ is a Gorenstein, Artin-Schelter regular algebra of dimension $n$. Let $\Psi \in A_{n}$ be a homogeneous central element which is not a zero divisor. Then there is a triangulated equivalence

$$
D_{\mathrm{gr}}^{\text {sing }}\left(A /\langle\langle\Psi\rangle) \cong D_{\mathrm{gr}}(A, \Psi) .\right.
$$

Proof. The proof is based on the fact that $A /\langle\Psi \Psi\rangle$ is an Auslander-Gorenstein algebra of dimension $n-1$, by [Le]. This ensures that an analogue of [Or1, Proposition 1.23] holds in our present noncommutative setting. The rest of the proof of [Or1, Theorem 3.9] then goes through, and Proposition 3.6 .3 follows.

Next, we apply [Or2, Theorem 2.5$]$ to the algebra $\mathfrak{B}(\Phi, \Psi)$. This way, we obtain a triangulated equivalence

$$
D_{\mathrm{gr}}^{\text {sing }}(\mathfrak{B}(\Phi, \Psi)) \cong D^{b}(\mathfrak{B}(\Phi, \Psi)) / \operatorname{Tails}(\mathfrak{B}(\Phi, \Psi)) .
$$

On the other hand, applying the equivalence of Artin and Van den Bergh, 3.5.5), and using the isomorphism in (3.5.4), we deduce that the quotient category on the right of 3.6.4 is equivalent to $D^{b} \operatorname{Coh}(\mathbb{E})$. This, combined with Proposition 3.6.3 yields part (i) of Theorem 3.6.2.

The proof of part (ii) is similar to the proof of the corresponding well-known result in commutative algebra, due to D. Eisenbud [Ei]. 
Example 3.6.5. One of the simplest examples is the case of a cubic curve $\mathbb{E}_{\tau} \subset \mathbb{P}^{2}=$ $\mathbb{P}\left(\mathbb{C}^{3}\right)$, with homogeneous equation of the form (cf. 2.3.4p)

$$
\psi^{\tau}(x, y, z):=x^{3}+y^{3}+z^{3}+\tau \cdot x y z, \quad \tau \in \mathbb{C}^{*} .
$$

Motivated by [ATV] and [LPP], to any point $u \in \mathbb{P}^{2}$ with homogeneous coordinates $(\alpha, \beta, \gamma)$, one associates the following $3 \times 3$-matrix $D$, as well as the corresponding adjoint $D^{\natural}$, the matrix formed by the $2 \times 2$-minors of $D$ :

$$
D:=\left(\begin{array}{ccc}
\alpha x & \beta z & \gamma y \\
\gamma z & \alpha y & \beta x \\
\beta y & \gamma x & \alpha z
\end{array}\right), \quad D^{\natural}=\left(\begin{array}{ccc}
\alpha^{2} y z-\beta \gamma x^{2} & \gamma^{2} x y-\alpha \beta z^{2} & \beta^{2} x y-\alpha \gamma y^{2} \\
\beta^{2} x y-\alpha \gamma z^{2} & \alpha^{2} y z-\beta \gamma y^{2} & \gamma^{2} y z-\alpha \beta x^{2} \\
\gamma^{2} x z-\alpha \beta y^{2} & \beta^{2} y z-\alpha \gamma x^{2} & \alpha^{2} x y-\beta \gamma z^{2}
\end{array}\right) .
$$

We have an identity $D \cdot D^{\natural}=D^{\natural} \cdot D=\operatorname{det} D \cdot \operatorname{Id}$. Assume that $\alpha, \beta, \gamma$ are all nonzero and put $D^{\prime}:=-\frac{1}{\alpha \beta \gamma} \cdot D^{\natural}$. Thus, we obtain an equation $D \cdot D^{\prime}=D^{\prime} \cdot D=-\frac{\operatorname{det} D}{\alpha \beta \gamma} \cdot$ Id.

Further, from the definition of $D$ one computes

$$
\operatorname{det} D=\left(\alpha^{3}+\beta^{3}+\gamma^{3}\right) x y z-\alpha \beta \gamma\left(x^{3}+y^{3}+z^{3}\right) .
$$

Therefore, assuming that the triple $(\alpha, \beta, \gamma)$ is such that $\alpha^{3}+\beta^{3}+\gamma^{3}=\tau \cdot \alpha \beta \gamma$, we may write $\operatorname{det} D=-\alpha \beta \gamma \cdot \psi^{\tau}$. We deduce that whenever $\psi^{\tau}(\alpha, \beta, \gamma)=0$ (cf. (3.6.6)), one has $D \cdot D^{\prime}=\psi^{\tau} \cdot \operatorname{Id}=D^{\prime} \cdot D$. This way, we have constructed a family of graded matrix factorizations

$$
M_{u}=\left(\mathbb{C}[x, y, z]^{\oplus 3} \underset{D}{\longleftarrow} \mathbb{D}\left[\mathbb{C}[x, y, z]^{\oplus 3}\right) \in D_{\mathrm{gr}}\left(\mathbb{C}[x, y, z], \psi^{\tau}\right), \quad u \in \mathbb{E}_{\tau},\right.
$$

parametrized by the points $u=(\alpha, \beta, \gamma) \in \mathbb{E}_{\tau}$ with nonvanishing coordinates.

There is an important class of point modules over the algebra $\mathfrak{A}(\Phi)$ introduced in [ATV]. A point module has a grading $P=\bigoplus_{k \geq 0} P^{(k)}$ such that $P^{(0)}=\mathbb{C}$ and $\operatorname{dim} P^{(k)}$ $\leq 1$ for any $k$. Given an integer $r>0$, we let $P_{\leq r}:=P / \bigoplus_{k>r} P^{(k)}$ denote the $r$ truncation of $P$.

Following [ATV], one proves that any point module $P$ is annihilated by $\Psi$, hence may be viewed as a $\mathfrak{B}(\Phi, \Psi)$-module. Further, it is not difficult to show that there exists $r>0$ such that the map $P \mapsto P_{\leq r}$ assigning to a point module its $r$-truncation gives a bijection between the moduli spaces of point modules and $r$-truncated point modules, respectively. Let $r_{o}$ be the minimal such $r$.

We expect that Example 3.6.5 can be generalized to a noncommutative setting. Specifically, let $\Phi$ be a homogeneous CY-potential of degree $d=a+b+c$, and let $\Psi \in \mathfrak{A}(\Phi)$ be a homogeneous central element of degree $d$.

Conjecture 3.6.8. To any point module $P$ over the algebra $\mathfrak{B}(\Phi, \Psi)$ one can associate naturally a matrix factorization $M(P)=\left(M_{+}, M_{-}\right)$, as in 3.6.1], where $\mathrm{rk} M_{ \pm}=$ $\operatorname{dim} P_{\leq r_{o}}$. 
In the $E_{6}$-case, one has $r_{o}=d-1=2$ and $\operatorname{dim} P_{\leq r_{o}}=d=3$. Moreover, it was shown in [ATV] that point modules are parametrized by the points of the corresponding elliptic curve $\mathbb{E}$. In that case, our conjectural matrix factorization $M(P)$ should reduce to 3.6.7), where $u \in \mathbb{E}$ stands for the parameter of the point module $P$.

\section{Three-dimensional Poisson structures}

\subsection{Some notation and conventions}

Given a (not necessarily smooth) finitely generated commutative $\mathbb{C}$-algebra $A$, write $\Omega^{1} A$ for the $A$-module of Kähler differentials of $A$, and let $\Omega^{*} A:=\Lambda_{A}^{*}\left(\Omega^{1} A\right)$ be the graded commutative algebra of differential forms, equipped with the de Rham differential $\mathrm{d}$. For each $p=1,2, \ldots$, we also have $\mathfrak{X}^{p} A=\operatorname{Hom}_{A}\left(\Omega^{p} A, A\right)$, the space of skew $p$ polyderivations $A \wedge \mathbb{C} \cdots \wedge_{\mathbb{C}} A \rightarrow A$.

Set $\mathfrak{X}^{0} A:=A=: \Omega^{0} A$. The graded space $\mathfrak{X}^{\bullet} A:=\bigoplus_{p \geq 0} \mathfrak{X}^{p} A$ has a natural structure of Gerstenhaber algebra with respect to the Schouten bracket $[-,-]: \mathfrak{X}^{p} A \times \mathfrak{X}^{q} A \rightarrow$ $\mathfrak{X}^{p+q-1} A$. Associated with a polyderivation $\eta \in \mathfrak{X}^{p} A$, there is a Lie derivative operator $L_{\eta}: \Omega^{\bullet} A \rightarrow \Omega^{\bullet-p+1} A$, resp. contraction operator $i_{\eta}: \Omega^{\bullet} A \rightarrow \Omega^{\bullet-p} A$. These operators make $\Omega^{\bullet} A$ a Gerstenhaber $\mathfrak{X}^{\bullet} A$-module.

Let $A=\mathbb{C}[\mathcal{M}]$ be the coordinate ring of a smooth affine variety $\mathcal{M}$, with tangent bundle $T_{\mathcal{M}}$, resp. cotangent bundle $T_{\mathcal{M}}^{*}$. Then we have canonical isomorphisms $\mathfrak{X}^{\bullet} A=$ $\Gamma\left(\mathcal{M}, \bigwedge^{\bullet} T_{\mathcal{M}}\right)$, resp. $\Omega^{\bullet} A=\Gamma\left(\mathcal{M}, \bigwedge^{\bullet} T_{\mathcal{M}}^{*}\right)$. We will also use the notation $\mathfrak{X}^{\bullet}(\mathcal{M})$, resp. $\Omega^{\bullet}(\mathcal{M})$, for these spaces.

\subsection{Unimodular Poisson structures}

Any Poisson bracket $\{-,-\}: A \times A \rightarrow A$ on a (not necessarily smooth) finitely generated commutative algebra $A$ determines (and is determined by) a bivector $\pi \in \mathfrak{X}^{2} A$, via the formula

$$
\{f, g\}:=\langle\mathrm{d} f \wedge \mathrm{d} g, \pi\rangle, \quad \forall f, g \in A .
$$

The Jacobi identity for the bracket $\{-,-\}$ is equivalent to the equation $[\pi, \pi]=0$ in $\mathfrak{X}^{3} A$.

Associated with any $f \in A$, there is a Hamiltonian derivation $\xi_{f}:=\{f,-\} \in \mathfrak{X}^{1} A$; it is easy to check that $\xi_{f}=[\pi, f]$.

Let $\mathcal{M}$ be a smooth affine variety of dimension $n$, with a trivial canonical bundle. Let vol $\in \Omega^{n}(\mathcal{M})$ be a nowhere vanishing volume $n$-form. Contraction with vol yields an isomorphism

$$
\mathfrak{X}^{p}(\mathcal{M}) \stackrel{\sim}{\rightarrow} \Omega^{n-p}(\mathcal{M}), \quad \eta \mapsto i_{\eta} \text { vol, } \quad p=0, \ldots, n .
$$

A Poisson bracket on the algebra $A=\mathbb{C}[\mathcal{M}]$ is said to be unimodular provided the divergence (with respect to the volume vol) of any Hamiltonian vector field vanishes, i.e., for any $f \in \mathbb{C}[\mathcal{M}]$, we have $\operatorname{div}\left(\xi_{f}\right)=\left(L_{\xi_{f}}\right.$ vol $) /$ vol $=0$. This means that the volume form is preserved by the Hamiltonian flow generated by the vector field $\xi_{f}$.

One has the following standard result. 
Lemma 4.2.3. Given an arbitrary bivector $\pi \in \mathfrak{X}^{2}(\mathcal{M})$, on a 3-dimensional smooth variety $\mathcal{M}$, let $\alpha:=i_{\pi}$ vol, a 1 -form. Then we have:

(i) The condition that $\pi$ be a Poisson bivector is equivalent to the equation $\alpha \wedge \mathrm{d} \alpha=0$.

(ii) $\pi$ gives a unimodular Poisson bracket $\Leftrightarrow L_{\pi} \mathrm{vol}=0 \Leftrightarrow \mathrm{d} \alpha=0$.

Proof. For any $\eta \in \mathfrak{X}^{p}(\mathcal{M})$, one has $i_{[\pi, \eta]}=\left[L_{\pi}, i_{\eta}\right]$, where $[-,-]$ stands for the super-commutator. Further, using Cartan's identity $L_{\pi}=i_{\pi} \mathrm{d}-\mathrm{d} i_{\pi}$ we get

$$
i_{[\pi, \eta]}=i_{\pi} \mathrm{d} i_{\eta}-\mathrm{d} i_{\pi} i_{\eta}-(-1)^{p} i_{\eta} i_{\pi} \mathrm{d}+(-1)^{p} i_{\eta} \mathrm{d} i_{\pi} .
$$

We take $p=2$ and apply the operations on each side of the identity to the 3-form vol. Clearly, $\mathrm{d}$ vol $=0$ and also $i_{\pi} i_{\eta} \mathrm{vol}=i_{\eta} i_{\pi} \mathrm{vol}=i_{\eta} \alpha=0$. Hence, we find

$$
i_{[\pi, \eta]} \mathrm{vol}=i_{\pi} \mathrm{d} i_{\eta} \mathrm{vol}+i_{\eta} \mathrm{d} \alpha+\mathrm{d} i_{\pi} \alpha=\left\langle\pi, \mathrm{d} i_{\eta} \mathrm{vol}\right\rangle+\langle\eta, \mathrm{d} \alpha\rangle .
$$

Now let $\eta=\pi$ and let $\Upsilon$ be the 3 -vector inverse to vol. Then $\pi=i_{\alpha} \Upsilon$. So, $\langle\pi, \mathrm{d} \alpha\rangle=$ $\left\langle i_{\alpha} \Upsilon, \mathrm{d} \alpha\right\rangle=\langle\Upsilon, \alpha \wedge \mathrm{d} \alpha\rangle$. Hence, we obtain $i_{[\pi, \pi]}$ vol $=2\langle\Upsilon, \alpha \wedge \mathrm{d} \alpha\rangle$. Thus, we see that $[\pi, \pi]=0$ if and only if $\alpha \wedge \mathrm{d} \alpha=0$. This yields part (i) since the pairing in 4.2.1) gives a Poisson bracket if and only if $[\pi, \pi]=0$.

There is also an alternative more geometric proof of (i) as follows. A bivector $\pi$ gives a Poisson structure on $\mathcal{M}$ if and only if $[\pi, \pi]=0$, which holds if and only if the distribution in $T_{\mathcal{M}}$ (of generic rank 2) spanned by $\pi$ is integrable. For $\alpha=i_{\pi}$ vol, the same distribution may be alternatively described as the distribution defined by the kernels of the 1 -form $\alpha$. The latter distribution is integrable if and only if $\alpha$ satisfies the Frobenius integrability condition $\alpha \wedge \mathrm{d} \alpha=0$.

The unimodularity property in part (ii) is equivalent to the equation

$$
0=\operatorname{div}\left(\xi_{f}\right) \cdot \operatorname{vol}=L_{\xi_{f}} \operatorname{vol}=\mathrm{d}\left(i_{\xi_{f}} \operatorname{vol}\right), \quad \forall f \in \mathbb{C}[\mathcal{M}] .
$$

We have

$$
\xi_{f}=i_{\mathrm{d} f} \pi=i_{\mathrm{d} f}\left(i_{\alpha} \Upsilon\right)=i_{\mathrm{d} f \wedge \alpha} \Upsilon .
$$

Therefore, we get $i_{\xi_{f}} \mathrm{vol}=\mathrm{d} f \wedge \alpha$, hence $\mathrm{d}\left(i_{\xi_{f}} \mathrm{vol}\right)=-\mathrm{d} f \wedge \mathrm{d} \alpha$. We see that 4.2.5 amounts to the equation $\mathrm{d} f \wedge \mathrm{d} \alpha=0$ for any regular function $f$. This holds if and only if $\mathrm{d} \alpha=0$.

\subsection{Unimodular Poisson structures on $\mathbb{C}^{3}$}

Fix a smooth 3-dimensional manifold with a nowhere vanishing volume form vol $\in$ $\Omega^{3}(\mathcal{M})$ and a regular function $\phi \in \mathbb{C}[\mathcal{M}]$.

Associated with $\mathrm{d} \phi$, an exact 1 -form, one has a bivector $\pi \in \mathfrak{X}^{2}(\mathcal{M})$ such that $i_{\pi} \mathrm{vol}=\mathrm{d} \phi$. By Lemma 4.2.3, this bivector gives rise to a unimodular Poisson bracket $\{-,-\}_{\phi}$ on $\mathbb{C}[\mathcal{M}]$. Explicitly, the bracket is determined by the equation

$$
\{f, g\}_{\phi} \cdot \operatorname{vol}=\mathrm{d} \phi \wedge \mathrm{d} f \wedge \mathrm{d} g, \quad \forall f, g \in \mathbb{C}[\mathcal{M}] .
$$

We now specialize to the case where $\mathcal{M}=\mathbb{C}^{3}$ is a vector space with coordinates $x, y, z$, and $\operatorname{vol}=\mathrm{d} x \wedge \mathrm{d} y \wedge \mathrm{d} z$ is the standard volume form. 
Corollary 4.3.2. Let $\{-,-\}$ be a unimodular polynomial Poisson structure on $\mathbb{C}[x, y, z]$. Then:

(i) There exists a polynomial $\phi \in \mathbb{C}[x, y, z]$ such that the Poisson bracket of linear functions is given by formula 1.3.1.

(ii) We have $\mathbb{C}[\phi] \subset \mathcal{Z}(\mathbb{C}[x, y, z])$. If the Poisson bracket is nonzero, then any element $f \in \mathcal{Z}(\mathbb{C}[x, y, z])$ is algebraic over the subalgebra $\mathbb{C}[\phi]$, i.e. there exists a nonzero polynomial $P \in \mathbb{C}\left[t_{1}, t_{2}\right]$ such that $P(\phi, f)=0$.

Proof. Recall that any polynomial closed 1 -form on $\mathbb{C}^{3}$ is exact. Hence, any unimodular Poisson bracket on the algebra $\mathbb{C}[\mathcal{M}]=\mathbb{C}[x, y, z]$ is of the form 4.3.1] for some polynomial function $\phi \in \mathbb{C}[\mathcal{M}]$. The corresponding Poisson bivector $\pi$ is given by

$\pi=i_{\mathrm{d} \phi} \Upsilon=\frac{\partial \phi}{\partial x} \cdot \frac{\partial}{\partial y} \wedge \frac{\partial}{\partial z}+\frac{\partial \phi}{\partial y} \cdot \frac{\partial}{\partial z} \wedge \frac{\partial}{\partial x}+\frac{\partial \phi}{\partial z} \cdot \frac{\partial}{\partial x} \wedge \frac{\partial}{\partial y}$, where $\Upsilon:=\frac{\partial}{\partial x} \wedge \frac{\partial}{\partial y} \wedge \frac{\partial}{\partial z}$.

Part (i) and the inclusion in part (ii) follow.

Next, let $f \in \mathbb{C}[x, y, z]$ be such that $\mathbb{C}(\phi, f)$, the field of rational functions generated by the polynomials $\phi$ and $f$, has transcendence degree 2 over $\mathbb{C}$. Then there exists a point $u \in \mathbb{C}^{3}$ such that $\left.\mathrm{d} \phi\right|_{u}$ and $\left.\mathrm{d} f\right|_{u}$ are linearly independent covectors.

Now, formula (4.3.1) shows that $f$ is a central element with respect to the Poisson bracket if and only if $\mathrm{d} \phi \wedge \mathrm{d} f=0$. Hence, for $f \in \mathcal{Z}(\mathbb{C}[x, y, z])$, the covectors $\left.\mathrm{d} \phi\right|_{u}$ and $\left.\mathrm{d} f\right|_{u}$ must be proportional, and part (ii) follows.

Remark 4.3.4. For a polynomial $\phi$ such that the ring $\mathbb{C}[\phi]$ is algebraically closed in $\mathbb{C}[x, y, z]$, Corollary 4.3.2 (ii) yields $\mathbb{C}[\phi]=\mathcal{Z}(\mathbb{C}[x, y, z])$. This condition holds for instance for any irreducible polynomial (cf. [P] Proposition 4.2] for a similar result in a special case).

\section{Poisson (co)homology}

\subsection{Poisson cohomology and the LKB complex}

Poisson homology $P H$. (A), resp. cohomology $P H^{\bullet}(A)$, of a Poisson algebra $A$ is defined as the homology of the total complex associated with a double complex, $D P_{\mathbf{.}}, .(A)=$ $\wedge_{A}^{\bullet}\left(D . \Omega^{1} A\right)$, resp. $D P^{\bullet \bullet}(A)=\operatorname{Hom}_{A}\left(D P_{\bullet}, \bullet(A), A\right)(\mathrm{cf}$. [GK, Appendix]). Here, $D . \Omega^{1} A$ denotes the cotangent complex of $A$, the latter being viewed as a commutative associative algebra (cf. [GK] formula (A.4)]).

The bigraded space $D P^{\bullet} \cdot(A)$ comes equipped with a natural Gerstenhaber (i.e. graded Poisson) algebra structure, of bidegree $(0,-1)$, which gives rise to a Gerstenhaber algebra structure on $P H^{\bullet}(A)$ (see [GK]). Also, $P H^{0}(A)=\mathcal{Z}(A)$ and for each $j=0,1, \ldots$, the group $P H^{j}(A)$, resp. $P H_{j}(A)$, comes equipped with a natural $\mathcal{Z}(A)$ module structure.

Let $\pi \in \mathfrak{X}^{2} A$ be the biderivation associated with the Poisson bracket (cf. (4.2.1)). The Lie derivative $L_{\pi}: \Omega^{\bullet} A \rightarrow \Omega^{\bullet-1} A$, resp. $L_{\pi}: \mathfrak{X}^{\bullet} A \rightarrow \mathfrak{X}^{\bullet+1} A$, makes the graded space 
$\Omega^{\bullet} A$, resp. $\mathfrak{X}^{\bullet} A$, a complex called the homological, resp. cohomological, LichnerowiczKoszul-Brylinski complex (LKB complex) of the Poisson algebra $A$ (cf. [ $[\mathrm{Br}]$ ).

The canonical projection $D . \Omega^{1} A \rightarrow \Omega^{1} A$ induces a map $D P_{\bullet}, \bullet(A) \rightarrow D P_{0, \bullet}(A)=$ $\Omega^{\bullet} A$, and also the dual map $\mathfrak{X}^{\bullet} A \rightarrow D P^{\bullet} \cdot \bullet(A)$. These maps provide morphisms between the LKB and Poisson cohomology complexes, respectively. Furthermore, unlike the case of the Hochschild complex, the map $\mathfrak{X}^{\bullet} A \rightarrow D P^{\bullet},{ }^{\bullet}(A)$ turns out to be a DG Gerstenhaber algebra morphism.

If the scheme Spec $A$ is smooth then the projection $D . \Omega^{1} A \rightarrow \Omega^{1} A$ is a quasiisomorphism. It follows that each of the morphisms $D P_{\bullet}, \bullet(A) \rightarrow \Omega^{\bullet} A$ and $\mathfrak{X}^{\bullet} A \rightarrow$ $D P^{\bullet} \cdot \bullet(A)$ is a quasi-isomorphism as well. In that case, Poisson (co)homology of $A$ may be computed via the corresponding LKB complex, that is (cf. [GK]), we have

$$
P H \cdot(A)=H^{\bullet}\left(\Omega^{\bullet} A, L_{\pi}\right), \quad \text { resp. } \quad P H^{\bullet}(A)=H^{\bullet}\left(\mathfrak{X}^{\bullet} A, L_{\pi}\right) .
$$

Observe that the de Rham differential $\mathrm{d}: \Omega^{\bullet} A \rightarrow \Omega^{\bullet+1} A$ anticommutes with the operator $L_{\pi}$, hence induces a well-defined operator $\mathrm{d}: P H_{\bullet}(A) \rightarrow P H_{\bullet+1}(A)$ on Poisson homology (cf. [X] $]$ ).

Assume next that $\operatorname{Spec} A$ is a manifold of pure dimension $n$, equipped with a nowhere vanishing volume form vol $\in \Omega^{n} A$. Define a differential $\delta: \mathfrak{X}^{\bullet} A \rightarrow \mathfrak{X}^{\bullet-1} A$ by transporting the de Rham differential $d: \Omega^{\bullet} A \rightarrow \Omega^{\bullet+1} A$ via the isomorphism $\mathfrak{X}^{\bullet} A \stackrel{\sim}{\rightarrow} \Omega^{n-\bullet} A$ (cf. (4.2.2). Then, by [Xu, Proposition 4.5 and Theorem 4.8], we have

Proposition 5.1.1. Let $\operatorname{Spec} A$ be smooth of pure dimension n. For any unimodular Poisson bivector $\pi \in \mathfrak{X}^{2} A$, one has:

(i) The isomorphism in 4.2.2 intertwines the $L_{\pi}$-actions on polyvector fields and on differential forms; it induces a degree reversing $\mathcal{Z}(A)$-module isomorphism $P H .(A)$ $\stackrel{\sim}{\rightarrow} P^{n-\bullet}(A)$.

(ii) The differential $\delta$ anticommutes with $L_{\pi}$; it descends to a well-defined $B V$-type differential $\delta: P H^{\bullet}(A) \rightarrow P H^{\bullet-1}(A)$.

\subsection{Poisson homology of a complete intersection}

Let $I \subset A$ be a Poisson ideal in a Poisson algebra $A$, so we have $\{I, A\} \subset I$. We set $B=A / I$. Thus, $B$ is a Poisson algebra and $\operatorname{Spec} B$ is a closed Poisson subscheme in Spec $A$.

The following is a Poisson analogue of a similar result known in the case of Hochschild cohomology (cf. e.g. [LR]).

Proposition 5.2.1. Assume that the Poisson scheme Spec A is smooth and, moreover, the Poisson subscheme $\operatorname{Spec} B$ is a locally complete intersection in Spec $A$. Then $L_{\pi}\left(I^{n} \cdot \Omega^{m} A\right) \subset I^{n+1} \cdot \Omega^{m-1} A$ for any $m, n \geq 0$, and there is a direct sum decomposition

$$
P H_{k}(B)=\bigoplus_{0 \leq 2 j \leq k} H^{k-2 j}\left(I^{j} \cdot \Omega^{\bullet} A / I^{j+1} \cdot \Omega^{\bullet} A, L_{\pi}\right), \quad \forall k \geq 0 .
$$


Proof. The first statement is verified by a direct computation. Further, the assumption that Spec $B$ be a locally complete intersection ensures that $I / I^{2}$ is a projective $B$-module, and the cotangent complex of Spec $B$ may be represented by a length two complex of amplitude $[-1,0]$,

$$
D . \Omega^{1} B \stackrel{\text { qis }}{\simeq}\left[I / I^{2} \stackrel{d}{\rightarrow} B \otimes_{A} \Omega^{1} A\right] .
$$

Hence, the Poisson double complex is quasi-isomorphic to a double complex with the following terms:

$$
\begin{aligned}
D P_{p, q}(B) \stackrel{\text { qis }}{\simeq} \Lambda_{B}^{q}\left(D_{p} \Omega^{1} B\right) & =\Lambda_{B}^{q}\left(\left[I / I^{2}\right][1] \oplus B \otimes_{A} \Omega^{1} A\right) \\
& =\bigoplus_{0 \leq j \leq q}\left(\left[\operatorname{Sym}^{j}\left(I / I^{2}\right)\right][j] \otimes_{A} \Omega^{q-j} A\right) \\
& =\bigoplus_{0 \leq j \leq q}\left(I^{j} \cdot \Omega^{q-j} A / I^{j+1} \cdot \Omega^{q-j} A\right)[j] .
\end{aligned}
$$

\subsection{Poisson cohomology of a hypersurface}

Below, we will be mostly interested in Poisson cohomology of an algebra of the form $B:=\mathbb{C}[\mathcal{M}] /(\phi)$ where $\mathcal{M}$ is a smooth Poisson variety and $\phi \in \mathbb{C}[\mathcal{M}]$ a regular function contained in the Poisson center. In that case, one can give a slightly different description of Poisson (co)homology of the algebra $B$, which is more explicit than the one provided by Proposition 5.2.1.

Observe first that contraction with the 1 -form $\mathrm{d} \phi$ provides a differential $i_{\mathrm{d} \phi}: \mathfrak{X}^{\bullet}(\mathcal{M})$ $\rightarrow \mathfrak{X}^{\bullet-1}(\mathcal{M})$ in the corresponding Koszul complex.

Remark 5.3.1. The Jacobi ring of $\phi$ may be identified with $H^{0}\left(\mathfrak{X}^{\bullet}(\mathcal{M}), i_{\mathrm{d} \phi}\right)$. The latter group is the only nontrivial cohomology group of the Koszul complex, provided $\phi$ has isolated singularities. This way, using the Euler-Poincaré principle, one proves formula 2.2.2.

Let $\pi \in \mathfrak{X}^{2}(\mathcal{M})$ be a Poisson bivector.

Lemma 5.3.2. For any $\phi \in \mathcal{Z}(\mathbb{C}[\mathcal{M}])$, the map $L_{\pi}$ is $\mathbb{C}[\phi]$-linear and it anticommutes with $i_{\mathrm{d} \phi}$.

Proof. In general, for any function $f$ and a bivector $\pi$, one has the standard identity

$$
L_{\pi} \circ i_{\mathrm{d} f}+i_{\mathrm{d} f} \circ L_{\pi}=L_{i_{\mathrm{d} f} \pi}=\xi_{f} .
$$

Now, the function $\phi$ is central with respect to the Poisson bracket given by a bivector $\pi$ if and only if $i_{\mathrm{d} \phi} \pi=0$. In that case the maps $L_{\pi}$ and $i_{\mathrm{d} \phi}$ anticommute. The $\mathbb{C}[\phi]$-linearity statement is clear.

Proposition 5.3.4. Let $\phi \in \mathcal{Z}(\mathbb{C}[\mathcal{M}])$ be a central regular function on $\mathcal{M}$. Assume that $\phi$ has only isolated singularities and that there exists a vector field eu $\in \mathfrak{X}^{1}(\mathcal{M})$ such that $\mathrm{eu}(\phi)=c \cdot \phi$, where $c$ is a nonzero constant. Then, for the Poisson cohomology of the algebra $B_{\phi}:=\mathbb{C}[\mathcal{M}] /(\phi)$, there is a convergent first quadrant spectral sequence $E_{2}^{p, q} \Rightarrow \operatorname{gr}_{p} P H^{p+q}\left(B_{\phi}\right)$, with $E_{1}$-term of the form 


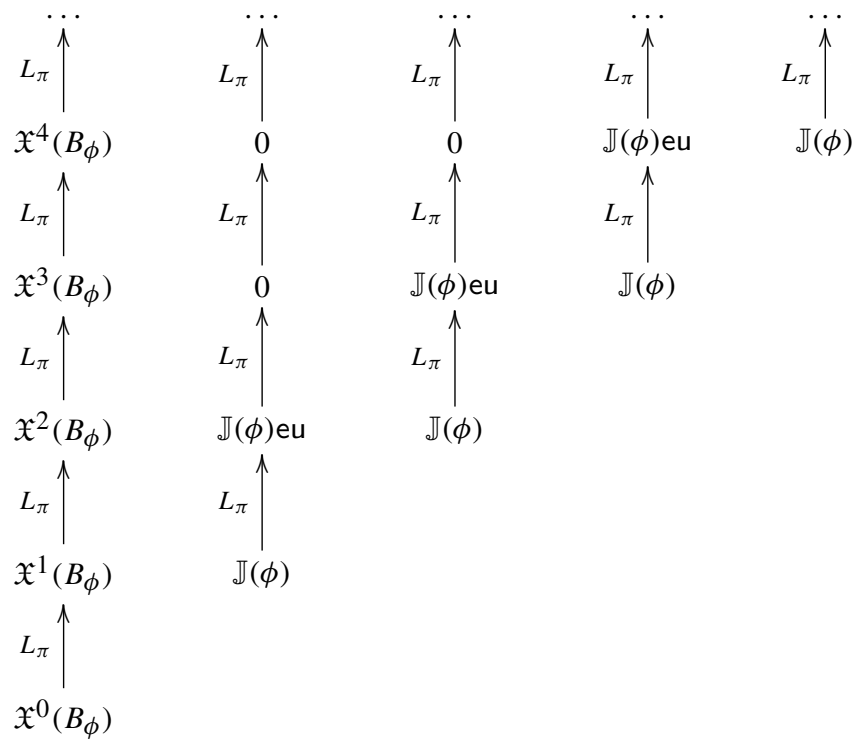

where the leftmost column is the LKB complex of the Poisson algebra $B_{\phi}$, and $\mathbb{J}(\phi)$ denotes the Jacobi ring of $\phi$ ( $c f$. $\$ 2.2$ ).

Proof. Put $A:=\mathbb{C}[\mathcal{M}]$ and let $\mathcal{D} A^{\bullet}=A \otimes \mathbb{C}[\tau] /\left(\tau^{2}\right)$ denote a graded super-commutative algebra such that $A$ is an even subalgebra placed in degree zero, and $\tau$ is an odd generator of degree -1 . We introduce a differential $\nabla: \mathcal{D} A^{\bullet} \rightarrow \mathcal{D} A^{\bullet+1}$, which is defined as an odd super-derivation, $\nabla=\phi \partial / \partial \tau$, that annihilates the subalgebra $A$ and is such that $\nabla(\tau)=\phi$. Clearly, one can view the resulting DG algebra as a 2-term complex $A \stackrel{\phi}{\longrightarrow} A$ with the differential given by multiplication by the function $\phi$. Therefore, we have $H_{0}(\mathcal{D} A)=B_{\phi}$ and $H_{j}(\mathcal{D} A)=0$ for any $j \neq 0$.

Next, we make $\mathcal{D} A$ a Poisson DG algebra by extending the Poisson bracket $\{-,-\}$ on $A$ by $\tau$-linearity. This way, $\mathcal{D} A$ becomes a Poisson DG algebra which is quasi-isomorphic to $B_{\phi}$. Thus, for the Poisson cohomology, we have $P H^{\bullet}\left(B_{\phi}\right) \cong P H^{\bullet}(\mathcal{D} A)$, where the cohomology on the right-hand side denotes the hyper-cohomology involving the differential $\nabla$, on $\mathcal{D} A$.

It will be convenient to use geometric language and write $\mathcal{D} A=\mathbb{C}[Y]$, where $Y=$ $\mathcal{M} \times \mathbb{C}$ is a smooth affine Poisson super-manifold of super-dimension $(\operatorname{dim} \mathcal{M} \mid 1)$. The corresponding Poisson cohomology may be computed, according to general principles, as a hyper-cohomology of the LKB double complex for the Poisson DG super-manifold $Y$. This way, we deduce

$$
P H^{\bullet}(A) \cong P H^{\bullet}(\mathcal{D} A) \cong H^{\bullet}\left(\mathfrak{X}^{\bullet} \mathcal{D} A, \nabla+L_{\pi}\right),
$$

where the differential $\nabla$ is induced by the same named differential on the DG algebra $\mathcal{D} A$ itself, and the differential $L_{\pi}$ comes from the Poisson structure on $\mathbb{C}[\mathcal{M}]$. 
Let $t$ denote an even coordinate on the total space, $T^{*} Y$, dual to the odd coordinate $\tau$ on $Y$. Thus, we get $\mathfrak{X}^{\bullet}(Y)=\mathfrak{X}^{\bullet}(\mathcal{M}) \otimes \mathbb{C}[t, \tau] /\left(\tau^{2}\right)$, where the variable $t$ is assigned grade degree +2 . With this notation, the LKB complex takes the form

$$
\left(\mathfrak{X}^{\bullet}(\mathcal{M}) \otimes \mathbb{C}[t, \tau] /\left(\tau^{2}\right), \phi \cdot \frac{\partial}{\partial \tau}+t \cdot i_{\mathrm{d} \phi}+L_{\pi}\right) .
$$

Let $T_{\phi}:=\left.T_{\mathcal{M}}\right|_{\phi^{-1}(0)}$ denote the restriction of the tangent bundle of $\mathcal{M}$ to the (not necessarily smooth) hyper-surface $\phi^{-1}(0) \subset \mathcal{M}$. Thus, $T_{\phi}$ is a vector bundle on $\phi^{-1}(0)$ of rank $\operatorname{dim} \mathcal{M}$, and we let $\Lambda_{\phi}^{\cdot}:=\Gamma\left(\phi^{-1}(0), \Lambda^{\bullet} T_{\phi}\right) \cong B_{\phi} \otimes_{A} \mathfrak{X}^{\bullet} A$ be the corresponding exterior algebra viewed as a graded algebra such that the space $B_{\phi} \otimes_{A} \mathfrak{X}^{1} A$ is placed in degree +1 .

Restriction to $\phi^{-1}(0)$ combined with the specialization $\tau \mapsto 0$ gives a natural algebra projection

$$
\operatorname{pr}:\left(\mathfrak{X}^{\bullet}(\mathcal{M}) \otimes \mathbb{C}[t, \tau] /\left(\tau^{2}\right), \phi \cdot \frac{\partial}{\partial \tau}+t \cdot i_{\mathrm{d} \phi}+L_{\pi}\right) \rightarrow\left(\Lambda_{\phi}^{\cdot} \otimes \mathbb{C}^{\bullet}[t], t \cdot i_{\mathrm{d} \phi}+L_{\pi}\right) .
$$

It is easy to see that the differential in 5.3.6 descends to the differential $t \cdot i_{\mathrm{d} \phi}$ on $\Lambda_{\phi}^{\cdot} \otimes$ $\mathbb{C}[t]$. Moreover, the map pr is a quasi-isomorphism of DG algebras.

Thus, we conclude that the Poisson cohomology of $B_{\phi}$ may be computed as hypercohomology of the DG algebra represented by the following mixed complex:

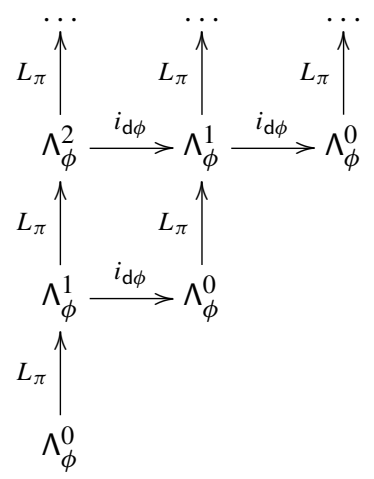

We view (5.3.8) as a bicomplex, $K$, with two differentials, $i_{\mathrm{d} \phi}$ and $L_{\pi}$. Associated with this bicomplex, there is a standard first quadrant spectral sequence $\left(E_{r}^{p, q}, d_{r}\right)$ such that $E_{1}=H^{\bullet}\left(K, i_{\mathrm{d} \phi}\right)$ and the differential $d_{1}$ is induced by $L_{\pi}$.

We first analyze the horizontal differential $i_{\mathrm{d} \phi}$. Let $\Lambda^{(q)}: \Lambda_{\phi}^{q} \rightarrow \cdots \rightarrow \Lambda_{\phi}^{1} \rightarrow \Lambda_{\phi}^{0}$ denote the complex in the $q$-th row of diagram (5.3.8) where, for any $j=0,1, \ldots, q$, the term $\Lambda_{\phi}^{j}$ is placed in degree $j$. The $E_{1}$ page of the spectral sequence of the bicomplex 5.3.8) takes the required form (5.3.5) thanks to the sublemma below.

Sublemma 5.3.9. (i) We have $H^{q}\left(\Lambda^{(q)}, i_{\mathrm{d} \phi}\right)=\mathfrak{X}^{q} B_{\phi}$.

(ii) The complex $\Lambda^{(q)}$ is acyclic in all degrees $j \neq 0,1$, or $q$. Moreover, $H^{0}\left(\Lambda^{(q)}, i_{\mathrm{d} \phi}\right)=$ $\mathbb{J}(\phi)$ and, if $q>1$, then also $H^{1}\left(\Lambda^{(q)}, i_{\mathrm{d} \phi}\right)=\mathbb{J}(\phi)$. 
Proof of Sublemma. To prove (i), recall that, in general, for any complete intersection $\mathcal{N} \subset \mathcal{M}$ where $\mathcal{M}$ is smooth, each polyderivation of the algebra $\mathbb{C}[\mathcal{N}]$ is induced by a section of the vector bundle $\left.\Lambda^{p} T_{\mathcal{M}}\right|_{\mathcal{N}}$. We take $\mathcal{N}=\mathcal{M}_{\phi}:=\phi^{-1}(0)$. It follows that each polyderivation $\theta: B_{\phi} \wedge \cdots \wedge B_{\phi} \rightarrow B_{\phi}$ comes from a section $s \in \Lambda_{\phi}^{p}=$ $\Gamma\left(\mathcal{M}_{\phi}, \Lambda^{p} T_{\mathcal{M}} \mid \mathcal{M}_{\phi}\right)$. An extension of $s$ to a section $\tilde{s} \in \Gamma\left(\mathcal{M}, \Lambda^{p} T_{\mathcal{M}}\right)$ gives a polyderivation $\widetilde{\theta}: A \wedge \cdots \wedge A \rightarrow A$ such that $\tilde{\theta}\left(\phi, a_{1}, \ldots, a_{p-1}\right) \in \phi \cdot A$ for any $a_{1}, \ldots, a_{p-1} \in A$. In geometric language, the latter condition translates into the equation $i_{\mathrm{d} \phi} s=0$, for the original section $s$. This proves (i).

Assume now that the function $\phi$ has an isolated singularity. Then the complex

$$
\mathfrak{X}^{(q)}: \mathfrak{X}^{q}(\mathcal{M}) \stackrel{i_{\mathrm{d} \phi}}{\longrightarrow} \mathfrak{X}^{q-1}(\mathcal{M}) \stackrel{i_{\mathrm{d} \phi}}{\longrightarrow} \cdots \longrightarrow \mathfrak{X}^{1}(\mathcal{M}) \stackrel{i_{\mathrm{d} \phi}}{\longrightarrow} \mathfrak{X}^{0}(\mathcal{M})
$$

is exact everywhere except possibly at the leftmost and rightmost terms. Furthermore, the cokernel at the rightmost term equals $\mathbb{J}(\phi)$.

By definition, we have a short exact sequence of complexes $0 \rightarrow \mathfrak{X}^{(q)} \rightarrow \mathfrak{X}^{(q)} \rightarrow$ $\Lambda^{(q)} \rightarrow 0$, where the morphism $\mathfrak{X}^{(q)} \rightarrow \mathfrak{X}^{(q)}$ is given by multiplication by the function $\phi$. From the corresponding long exact sequence of cohomology, we deduce that $H^{j}\left(\Lambda^{(q)}, i_{\mathrm{d} \phi}\right)=0$ unless $j \neq 0,1, q$. Moreover, since $H^{0}\left(\mathfrak{X}^{(q)}, i_{\mathrm{d} \phi}\right)=\mathbb{J}(\phi)$, the final part of the long exact sequence reads

$$
0=H^{1}\left(\mathfrak{X}^{(q)}, i_{\mathrm{d} \phi}\right) \rightarrow H^{1}\left(\Lambda^{(q)}, i_{\mathrm{d} \phi}\right) \rightarrow \mathbb{J}(\phi) \stackrel{\phi}{\rightarrow} \mathbb{J}(\phi) \rightarrow H^{0}\left(\Lambda^{(q)}, i_{\mathrm{d} \phi}\right) \rightarrow 0 .
$$

Now, by our assumptions, we have $\phi \in \mathbb{C} \cdot i_{\mathrm{d} \phi}$ eu. Therefore, the image of $\phi$ in the Jacobi ring $\mathbb{J}(\phi)$ vanishes. Thus, the map $\mathbb{J}(\phi) \rightarrow \mathbb{J}(\phi)$ induced by multiplication by $\phi$ is equal to zero. This, combined with the exact sequence 5.3.10), yields part (ii) of the sublemma. In addition, an easy diagram chase shows that the preimage of the element $1 \in \mathbb{J}(\phi)$ under the isomorphism $H^{1}\left(\Lambda^{(q)}, i_{\mathrm{d} \phi}\right) \stackrel{\sim}{\rightarrow} \mathbb{J}(\phi)$ (cf. 5.3.10) $)$ corresponds to the class of the vector field eu.

\subsection{Poisson cohomology of the algebra $\mathscr{A}_{\phi}$}

We now specialize to the setting of $\$ 2.2$. Thus, let $0<a \leq b \leq c$ be a triple of integers with $\operatorname{gcd}(a, b, c)=1$. Write eu for the corresponding Euler vector field 2.2.1) on $\mathcal{M}=$ $\mathbb{C}^{3}$, and $\Upsilon$ for the standard 3-vector (see 4.3 .3 ).

Given a homogeneous polynomial $\phi \in \mathbb{C}[\mathcal{M}]=\mathbb{C}[x, y, z]$, write $\pi:=i_{\mathrm{d} \phi} \Upsilon(\mathrm{cf}$. (4.3.3), and let $\mathscr{A}_{\phi}$ denote the corresponding Poisson algebra (cf. Definition 1.3.2).

A. Pichereau has found all Poisson cohomology groups of the algebra $\mathscr{A}_{\phi}$ explicitly (see $[\mathrm{P}]$ ). To state some of her results set $\mu:=\operatorname{dim} \mathbb{J}(\phi)$ and choose homogeneous elements $1, f_{1}, \ldots, f_{\mu-1} \in \mathbb{C}[x, y, z]$ such that their residue classes modulo the Jacobi ideal form a $\mathbb{C}$-basis of the vector space $\mathbb{J}(\phi)$. View the elements $i_{\mathrm{d} f_{k}} \Upsilon \in \mathfrak{X}^{2}\left(\mathbb{C}^{3}\right), k=$ $1, \ldots, \mu-1$, as elements of the LKB complex for the algebra $\mathscr{A}_{\phi}$.

Proposition 5.4.1 (Pichereau). For any homogeneous polynomial $\phi$ with an isolated singularity of degree $a+b+c$, the Poisson cohomology of $\mathscr{A}_{\phi}$ vanishes in degrees $\geq 4$, and one has: 
(i) $P H^{0}\left(\mathscr{A}_{\phi}\right)=\mathbb{C}[\phi]$. Furthermore, the group $P H^{1}\left(\mathscr{A}_{\phi}\right)=\mathbb{C}[\phi]$ eu is a rank 1 free $\mathbb{C}[\phi]$-module with generator eu.

(ii) The group $P H^{3}\left(\mathscr{A}_{\phi}\right)$ is a rank $\mu$ free $\mathbb{C}[\phi]$-module with basis $\Upsilon, f_{1} \cdot \Upsilon, \ldots, f_{\mu-1} \cdot \Upsilon$, resp. $P H^{2}\left(\mathscr{A}_{\phi}\right)$ is a free $\mathbb{C}[\phi]$-module with basis $i_{\mathrm{d} f_{1}} \Upsilon, \ldots, i_{\mathrm{d} f_{\mu-1}} \Upsilon, \pi$.

\subsection{Poisson cohomology of a quasi-homogeneous singularity}

Pichereau has also computed cohomology groups of the LKB complex for the (singular) Poisson algebra $\mathscr{B}_{\phi}$ associated with a quasi-homogeneous polynomial $\phi \in \mathbb{C}[x, y, z]$ of an arbitrary weight $d>0$. Specifically, she shows that $\mathfrak{X}^{p} \mathscr{B}_{\phi}=0$ for all $p>2$. Furthermore, the cohomology of the LKB-differential $L_{\pi}$ is as follows (see $[\mathrm{P}]$ ):

$$
H^{0}\left(\mathfrak{X}^{\bullet} \mathscr{B}_{\phi}\right)=\mathbb{C}, \quad H^{1}\left(\mathfrak{X}^{\bullet} \mathscr{B}_{\phi}\right)=\mathbb{J}^{(\varpi)}(\phi) \text { eu }, \quad H^{2}\left(\mathfrak{X}^{\bullet} \mathscr{B}_{\phi}\right) \cong \mathbb{J}^{(\varpi)}(\phi) \pi,
$$

where $\mathbb{J}^{(\varpi)}(\phi)$ is viewed, in the notation of the previous subsection, as the span of the basis elements $f_{j}$ with $\operatorname{deg} f_{j}=\varpi$.

Proof of Theorem 2.4.1. We begin with part (ii) of the theorem. Our Poisson bivector has the form $\pi=i_{\mathrm{d} \phi} \Upsilon$. Therefore, for any $f \in \mathscr{A}_{\phi}$ we have $L_{\pi}(f)=\xi_{f}=i_{\mathrm{d} f \wedge \mathrm{d} \phi} \Upsilon \subset$ $i_{\mathrm{d} \phi}\left(\mathfrak{X}^{2}\left(\mathscr{A}_{\phi}\right)\right)$. It follows that, for any $p \geq 1$, the vertical differential $L_{\pi}: E_{1}^{p, p}=$ $\mathbb{J}(\phi) \rightarrow E_{1}^{p, p+1}=\mathbb{J}(\phi)$ eu, in the spectral sequence 5.3 .5 , vanishes. Thus, the $E_{2}$ page of the spectral sequence reads

$$
\begin{array}{ccccc}
\ldots & \ldots & \ldots & \ldots & \ldots \\
H^{4}\left(\mathfrak{X}^{\bullet} \mathscr{B}_{\phi}, L_{\pi}\right) & 0 & 0 & \mathbb{J}(\phi) \text { eu } & \mathbb{J}(\phi) \\
H^{3}\left(\mathfrak{X}^{\bullet} \mathscr{B}_{\phi}, L_{\pi}\right) & 0 & \mathbb{J}(\phi) \text { eu } & \mathbb{J}(\phi) & \\
H^{2}\left(\mathfrak{X} \cdot \mathscr{B}_{\phi}, L_{\pi}\right) & \mathbb{J}(\phi) \text { eu } & \mathbb{J}(\phi) & & \\
H^{1}\left(\mathfrak{X} \cdot \mathscr{B}_{\phi}, L_{\pi}\right) & \mathbb{J}(\phi) & & & \\
H^{0}\left(\mathfrak{X}^{\bullet} \mathscr{B}_{\phi}, L_{\pi}\right) & & & &
\end{array}
$$

Here, the cohomology in the leftmost column is provided by formula 5.5.1], hence vanish in degrees $>2$. Thus, we see that all differentials $d_{r}: E_{r}^{p, q} \rightarrow E_{r}^{p-r+1, q+r}$, $r \geq 3$, have zero range, and the statement of part (ii) follows.

The case of Hochschild cohomology is quite similar. Write $H^{\bullet}(-)=H H^{\bullet}(-, b)$, where we have explicitly indicated the Hochschild differential $b$. Then, using the notation $\nabla=\phi \frac{\partial}{\partial \tau}$, we get

$$
H H^{\bullet}\left(\mathscr{B}_{\phi}, b\right)=H H^{\bullet}(\mathcal{D} A, \nabla+b)=H H^{\bullet}(\mathbb{C}[Y], \nabla+b)=H^{\bullet}\left(\mathfrak{X}^{\bullet}(Y), \nabla\right),
$$

where the last isomorphism is due to the Hochschild-Kostant-Rosenberg theorem applied to the smooth super-scheme $Y$.

One can now repeat the argument in the proof of Proposition 5.3.4 and replace the complex $\left(\mathfrak{X}^{\bullet}(Y), \nabla\right)$ by a smaller complex $\left(\wedge_{\phi}^{*} \otimes \mathbb{C}[t], t \cdot i_{\mathrm{d} \phi}\right)$, which is quasi-isomorphic to it (cf. (5.3.7)). This way, we see that the Hochschild cohomology of the algebra $\mathscr{B}_{\phi}$ may be computed as hyper-cohomology of the complex similar to (5.3.8), where the vertical differential $L_{\pi}$ is replaced by zero. This yields part (i). 
Remark 5.5.3. (i) Theorem 2.4.1 shows that the Poisson cohomology groups of the algebra $\mathscr{B}_{\phi}$ are nonzero in all degrees $\geq 2$, in particular, these groups are not the same as the cohomology groups of the LKB complex (cf. [5.5.1)). That agrees with the fact that the surface $\mathcal{M}_{\phi}=\operatorname{Spec} \mathscr{B}_{\phi}$ has a singularity.

(ii) Let $f \in \mathscr{A}_{\phi}$. For any $p=1,2, \ldots$, the image of the element $f$ in $\mathbb{J}(\phi)$ gives a class in $E_{2}^{p, p}$ (cf. (5.5.2). An explicit lift of that class to a $2 p$-cocycle in the total complex associated with the corresponding bicomplex $(5.3 .8)$ is provided by the element $f+i_{\mathrm{d} f} \Upsilon \in \Lambda_{\phi}^{0} \oplus \Lambda_{\phi}^{2}$. Indeed, we have $L_{\pi} f=\xi_{f}=i_{\mathrm{d} f \wedge \mathrm{d} \phi} \Upsilon$ (cf. 4.2.6). Further, using [5.3.3, the fact that $L_{\pi} \Upsilon=0$ and unimodularity of the Poisson bivector $\pi$ (Lemma 4.2.3, we get $L_{\pi} i_{\mathrm{d} f} \Upsilon=L_{i_{\mathrm{d} f} \pi} \Upsilon=L_{\xi_{f}} \Upsilon=0$. Thus, we compute

$\left(L_{\pi}+i_{\mathrm{d} \phi}\right)\left(f+i_{\mathrm{d} f} \Upsilon\right)=L_{\pi} f+L_{\pi} i_{\mathrm{d} f} \Upsilon+i_{\mathrm{d} \phi} i_{\mathrm{d} f} \Upsilon=i_{\mathrm{d} f \wedge \mathrm{d} \phi} \Upsilon+0+i_{\mathrm{d} \phi \wedge \mathrm{d} f} \Upsilon=0$.

Similarly, for a homogeneous function $f \in \mathscr{A}_{\phi}$ of degree $\operatorname{deg} f=k$, the element $(\varpi-k) f \Upsilon+f$ eu $\in \Lambda_{\phi}^{3} \oplus \Lambda_{\phi}^{1}$ gives, for each $p=1,2, \ldots$, a $(2 p+1)$-cocycle in the total complex associated with the corresponding bicomplex (5.3.8). To see this, one may use the identity $\pi \wedge \mathrm{eu}=\operatorname{deg} \phi \cdot \Upsilon$ to obtain the following equation (see [ $[\mathrm{P}$, formula (27)]):

$$
L_{\pi}(f \mathrm{eu})=(k-\varpi) f \pi-\operatorname{deg} \phi \cdot \phi \cdot i_{\mathrm{d} f} \Upsilon=(k-\varpi) f \pi \bmod (\phi) .
$$

Further, we have $i_{\mathrm{d} \phi} \mathrm{eu}=\mathrm{eu}(\phi)=(a+b+c) \phi$. Thus, we find that, in $\Lambda_{\phi}^{\cdot}=$ $\mathfrak{X}^{\bullet}(\mathcal{M}) /(\phi)$, one has

$$
\begin{aligned}
\left(L_{\pi}+i_{\mathrm{d} \phi}\right)[(\varpi-k) f \Upsilon+f \mathrm{eu}] & =(\varpi-k) f i_{\mathrm{d} \phi} \Upsilon+L_{\pi}(f \mathrm{eu})+f \mathrm{eu}(\phi) \\
& =(\varpi-k) f \pi+(k-\varpi) f \pi \bmod (\phi)=0 .
\end{aligned}
$$

\section{Classification results}

\subsection{Proof of Proposition 2.3 .2}

Assume that the curve is not rational. Let $a \leq b \leq c$.

If all the degrees are equal, then they are equal to 1 , and $\operatorname{deg} \phi \leq 3$. In this case, the statement is classical (the $E_{6}$ case).

Now assume that the degrees are not equal to each other. In this case the leading power of $z$ is $\leq 2$. If this power were 1 , the curve would be rational, so it is 2 . Consider two cases.

Case 1: $a<b=c$. In this case $z^{2}$ comes together with $z y$ and $y^{2}$, so for generic coefficients, by making a linear change of $y, z$, we can kill $z^{2}$ and $y^{2}$, so the leading term in $z$ will be linear. This shows that the curve is rational, a contradiction.

Case 2: $a \leq b<c$. Then the leading term in $z$ is $z^{2}$, so we get $2 c \leq a+b+c$, hence $c \leq a+b$. After a change of variable the equation of the curve can be written as $z^{2}=g(x, y)$, where $g$ is homogeneous of degree $2 c \leq 2(a+b)$. Consider two cases.

Case 2a: $a=b$. In this case, $g$ has degree 3 or 4 . If the degree of $g$ is 3 , then $a=$ $b=2, c=3$, and then the curve is rational, because the point $(x, y, z)$ is equivalent to 
$(x, y,-z)$ in the weighted projective space. Thus it remains to consider the case when $\operatorname{deg} g=4$, and thus $a=b=1, c=2$. In this case, for generic coefficients we do get an elliptic curve (the $E_{7}$ case).

Case 2b: $a<b$. Then the terms that can be present in $g(x, y)$ are $y^{3}$ and terms that contain $y$ in power $\leq 2$. Thus for the curve not to be rational, the term $y^{3}$ must be present. So $2 c=3 b$, and thus $b \leq 2 a$. If $b=2 a$, then $a=1, b=2, c=3, d=6$, and for generic coefficients we indeed get an elliptic curve (the $E_{8}$ case). On the other hand, if $b<2 a$, then $g$ cannot contain quadratic terms in $y$ (the only possible quadratic terms are $y^{2}, x y^{2}, x^{2} y^{2}$, and none of them has the right degree). The only linear term in $y$ that can occur in $g$ is $x^{3} y$, in which case the curve is given by $z^{2}=y^{3}+x^{3} y$, in weighted projective space of weights $(4,6,9)$. This curve is rational, because the point $(x, y, z)$ is equivalent to $(x, y,-z)$. Otherwise, the curve is $z^{2}=y^{3}+x^{p}, 4 \leq p \leq 5$, in weighted projective space with weights $(6,2 p, 3 p)$. If $p=5$, the curve is rational since $(x, y, z)$ is equivalent to $(x, y,-z)$. If $p=4$, the curve is given by the equation $z^{2}=y^{3}+x^{4}$, with weights $(3,4,6)$, and the curve is rational since $(x, y, z)$ is equivalent to $(x, \varepsilon y, z)$, where $\varepsilon$ is a cubic root of unity.

\subsection{Proof of Theorem 3.4.5 1)}

Let $Y^{\prime}$ be the space of all nonhomogeneous potentials of degree $a+b+c$, and $Y$ be the space of all nonhomogeneous commutative polynomials of that degree. Let $G^{\prime}$ be the group of degree preserving automorphisms of $\mathbb{C}\langle x, y, z\rangle$. Then we have the following exact sequence of $G^{\prime}$-modules:

$$
0 \rightarrow U \rightarrow Y^{\prime} \rightarrow Y \rightarrow 0,
$$

where $U$ is a 1-dimensional representation spanned by $x y z-y x z$ in the $E_{6}$ case, and a 2-dimensional representation spanned by $x y z-y x z$ and $x y x y-x^{2} y^{2}$ in the $E_{7}$ and $E_{8}$ cases.

Also, let $G$ be the group of degree preserving automorphisms of $\mathbb{C}[x, y, z]$. We have an exact sequence

$$
1 \rightarrow H \rightarrow G^{\prime} \rightarrow G \rightarrow 1,
$$

where $H=1$ in the $E_{6}$ case, and $H=\mathbb{G}_{a}$ consisting of elements $x \rightarrow x, y \rightarrow y, z \rightarrow$ $z+b(x y-y x)$ in the $E_{7}$ and $E_{8}$ cases. It is easy to see that a generic element of $U$ is equivalent under $H$ to $\gamma(x y z-y x z)$. Thus to prove the theorem, it suffices to show that the expressions $x y z+c \cdot[P+Q+R]$ (cf. $[2.5 .2)$ ) give normal forms of generic elements in $Y$ under the action of $G$. But this is a classical fact from the theory of del Pezzo surfaces (see $[\bar{D}]$ ).

\section{Calabi-Yau deformations}

\subsection{The $D G$ algebra $\mathfrak{D}(\Phi)$}

Let $F=\mathbb{C}\left\langle x_{1}, \ldots, x_{n}\right\rangle$ be a free algebra on $n$ homogeneous generators $x_{1}, \ldots, x_{n}$, where $\operatorname{deg} x_{i}>0$ for all $i=1, \ldots, n$. We view $F$ either as a graded or as a filtered algebra, as in $\$ 3.2$. We shall refer to the grading on $F$ as a weight grading. 
Associated with any potential $\Phi \in F_{\text {cyc }}$, we have introduced in [Gi §1.4] a free graded associative algebra $\mathfrak{D}(\Phi)=\bigoplus_{r \geq 0} \mathfrak{D}(\Phi)_{r}$ with $2 n+1$ homogeneous generators $x_{1}, \ldots, x_{n}, y_{1}, \ldots, y_{n}, t$. We have $\mathfrak{D}(\Phi)_{0}=F$. The algebra $\mathfrak{D}(\Phi)$ comes equipped with a differential $\partial: \mathfrak{D}(\Phi) . \rightarrow \mathfrak{D}(\Phi) \bullet_{-1}$ such that $H^{0}(\mathfrak{D}(\Phi))=F / \partial\left(\mathfrak{D}_{1}(\Phi)\right)=\mathfrak{A}(\Phi)$.

In the case where $\Phi$ is a homogeneous potential of degree $d>\max \left\{\operatorname{deg} x_{i} \mid i=\right.$ $1, \ldots, n\}$, there is an additional weight grading on $\mathfrak{D}(\Phi)$ such that the generators $y_{1}, \ldots$, $y_{n}, t$ are assigned degrees $\operatorname{deg} y_{i}:=d-\operatorname{deg} x_{i}$, and $\operatorname{deg} t:=d$. This way, multiplication by elements of $\mathfrak{D}(\Phi)_{0}$ makes each component $\mathfrak{D}(\Phi)_{r}$ a graded left $F$-module $\mathfrak{D}(\Phi)_{r}=$ $\bigoplus_{s>0} \mathfrak{D}^{(s)}(\Phi)_{r}$, where the $s$-grading denotes the weight grading.

The precise definition of the DG algebra $\mathfrak{D}(\Phi)$ is not essential for us at the moment. The important points are the following four properties:

- The differential on $\mathfrak{D}(\Phi)$ and the weight grading are determined by the potential $\Phi$, while the algebra structure and the $r$-grading do not involve the potential.

- For each $r=0,1, \ldots$, the homogeneous component $\mathfrak{D}(\Phi)_{r}$ is a free $F$ module, and if $\Phi$ is homogeneous, then $\operatorname{dim}_{\mathbb{C}}\left(\mathfrak{D}^{(s)}(\Phi)_{r}\right)<\infty, \forall s \geq 0$.

- If $H^{j}(\mathfrak{D}(\Phi))=0$ for all $j>0$, then $\Phi$ is a CY-potential.

- If $\Phi$ is a homogeneous CY-potential then the differential $\partial$ preserves the weight grading on $\mathfrak{D}(\Phi)$; moreover, $H^{j}(\mathfrak{D}(\Phi))=0$ for all $j>0$, i.e. the converse to 7.1 .3 holds as well.

Here, 7.1.1 -7.1 .2 are immediate from the definition of $\mathfrak{D}(\Phi)$, while $7.1 .3-7.1 .4$ follow from [Gi, Theorem 5.3.1], which is one of the main results of that paper.

For each $i=1, \ldots, n$, write $d_{i}:=\operatorname{deg} x_{i}>0$, and let Aut $F$ denote the group of degree preserving automorphisms of the algebra $F$. Given $d \geq 3$, let $\mathrm{CY}_{3}\left(d, d_{1}, \ldots, d_{n}\right) \subset$ $F_{\text {cyc }}^{(d)}$ be the set of homogeneous CY-potentials of some fixed degree $d \geq 3$.

Lemma 7.1.5. (i) The set $\mathrm{CY}_{3}\left(d, d_{1}, \ldots, d_{n}\right)$ is Aut $F$-stable; moreover, it is the intersection of an at most countable family of Zariski open (possibly empty) subsets in $F_{\text {cyc }}^{(d)}$.

(ii) For all $\Phi \in \mathrm{CY}_{3}\left(d, d_{1}, \ldots, d_{n}\right)$, the algebras $\mathfrak{A}(\Phi)$ have the same Hilbert-Poincaré series.

Proof. For any $\Phi \in F_{\text {cyc }}^{(d)}$, we may split the differential $\partial$ on the DGA $\mathfrak{D}(\Phi)$ into components $\partial_{r, s}^{\Phi}: \mathfrak{D}^{(s)}(\Phi)_{r} \rightarrow \mathfrak{D}^{(s)}(\Phi)_{r-1}$, where each $\mathfrak{D}^{(s)}(\Phi)_{r}$ is a finite-dimensional vector space, by (7.1.2). Since $\partial^{2}=0$, for any $r, s \geq 0$ one has dim Image $\partial_{r+1, s}^{\Phi} \leq \operatorname{dim} \operatorname{Ker} \partial_{r, s}^{\Phi}$.

According to property (7.1.4), $\Phi$ is a CY-potential iff the DGA $\mathfrak{D}(\Phi)$ has no nonzero cohomology in positive degrees. Thus, we have

$$
\mathrm{CY}_{3}\left(d, d_{1}, \ldots, d_{n}\right)=\left\{\begin{array}{l|l}
\Phi \in F_{\mathrm{cyc}}^{(d)} & \begin{array}{l}
\operatorname{dim} \text { Image } \partial_{r+1, s}^{\Phi} \geq \operatorname{dim} \operatorname{Ker} \partial_{r, s}^{\Phi}, \text { and } \\
\partial_{1, s}^{\Phi} \text { has maximal rank, } \forall r>0, s \geq 0
\end{array}
\end{array}\right\}
$$

The set on the right is clearly the intersection of a countable family of Zariski open subsets in $F_{\text {cyc }}^{(d)}$. Part (i) follows. Part (ii) is [Gi, Proposition 5.4.7]. 


\subsection{Deformation setup}

In this and the following subsection, we develop a formalism that will be used in the proofs of our main results.

Given a vector space $V$, we write $V[[\hbar]]$ for the space of formal power series in an indeterminate $\hbar$ with coefficients in $V$. In particular, we have $\mathbb{C}[[\hbar]]$, the ring of formal power series. A $\mathbb{C}[[\hbar]]$-module is said to be topologically free if it is isomorphic to a module of the form $V[[\hbar]]$, where $V$ is a $\mathbb{C}$-vector space. Such a module is clearly a flat $\mathbb{C}[[\hbar]]$-module, complete in the $\hbar$-adic topology.

Let $K=\bigoplus_{r \geq 0} K_{r}$ be a complex of topologically free $\mathbb{C}[[\hbar]]-$ modules, equipped with a $\mathbb{C}[[\hbar]]-$ linear continuous differential $d: K \cdot \rightarrow K \cdot{ }_{\bullet-1}$. Put $\bar{K}:=K / \hbar \cdot K$. This is a complex of $\mathbb{C}$-vector spaces, with induced differential $\bar{d}: \bar{K} \cdot \rightarrow \bar{K}_{\bullet-1}$.

We recall the following standard result.

Lemma 7.2.1. If the complex $(\bar{K}, \bar{d})$ is acyclic in positive degrees then:

(i) The complex $(K, d)$ is acyclic in positive degrees.

(ii) The cohomology group $H^{0}(K, d)$ is a flat $\mathbb{C}[[\hbar]]-m o d u l e$.

(iii) The projection $K \rightarrow \bar{K}$ induces an isomorphism

$$
H^{0}(K, d) / \hbar \cdot H^{0}(K, d) \stackrel{\sim}{\rightarrow} H^{0}(\bar{K}, \bar{d}) .
$$

We will also use a graded analogue of the above lemma, where the variable $\hbar$ is assigned grade degree 1 . Thus, let $K$ be a complex of graded $\mathbb{C}[\hbar]$-modules $K_{r}=\bigoplus_{s \geq 0} K_{r}^{s}$, with homogeneous, $\mathbb{C}[\hbar]$-linear differential $d: K_{r} \rightarrow K_{r-1}$. Put $\bar{K}:=K / \hbar K$, resp. $K^{\prime}:=K /(\hbar-1) K$, and let $\bar{d}$, resp. $d^{\prime}$, be the induced differential on $\bar{K}$, resp. on $K^{\prime}$. For each $r$, the grading on $K_{r}$ induces a filtration on $K_{r}^{\prime}$. Replacing each term $K_{r}$ by its completion $\widehat{K}_{r}:=\prod_{s \geq 0} K_{r}^{s}$ and applying Lemma 7.2.1 to the resulting complex yields the following elementary result.

Lemma 7.2.2. Assume, in the above setting, that each $K_{r}$ is a free graded $\mathbb{C}[\hbar]$-module such that $\operatorname{dim}_{\mathbb{C}} K_{r}^{s}<\infty$ for all $r, s$, and that $H^{r}(\bar{K}, \bar{d})=0$ for any $r>0$. Then $H^{r}\left(K^{\prime}, d^{\prime}\right)=0$ for all $r>0$. Furthermore, the natural map $\bar{K} \rightarrow \operatorname{gr} K^{\prime}$ induces an isomorphism $H^{0}(\bar{K}, \bar{d}) \stackrel{\sim}{\rightarrow} \operatorname{gr} H^{0}\left(K^{\prime}, d^{\prime}\right)$.

Below, it will be necessary to work with $\mathbb{C}[[\hbar]]-$ algebras, that is, with associative algebras $B$ equipped with a central algebra imbedding $\mathbb{C}[[\hbar]] \hookrightarrow B$. A $\mathbb{C}[[\hbar]]-$ algebra $B$ which is complete in the $\hbar$-adic topology will be referred to as an $\hbar$-algebra. Abusing terminology, we call such an algebra flat if it is topologically free as a left (equivalently, right) $\mathbb{C}[[\hbar]]$-module.

We reserve the notation $F_{\hbar}$ for the $\hbar$-algebra $F[[\hbar]]$. We have a canonical isomorphism of free $\mathbb{C}[[\hbar]]$-modules $F_{\hbar} /\left[F_{\hbar}, F_{\hbar}\right] \cong F_{\text {cyc }}[[\hbar]]$. This way, for any potential

$$
\Phi=\Phi_{0}+\hbar \cdot \Phi_{1}+\hbar^{2} \cdot \Phi_{2}+\cdots \in(F[[\hbar]])_{\mathrm{cyc}}=F_{\mathrm{cyc}}[[\hbar]],
$$

where $\Phi_{j} \in F_{\text {cyc }}$, one may define the $\hbar$-algebras

$$
\mathfrak{A}_{\hbar}(\Phi):=F_{\hbar} /\left\langle\left\langle\partial_{i} \Phi\right\rangle\right\rangle_{i=1, \ldots, n}, \quad \text { resp. } \quad \mathfrak{D}_{\hbar}(\Phi)=\bigoplus_{r \geq 0} \mathfrak{D}_{\hbar}(\Phi)_{r} .
$$


Here, $\mathfrak{D}_{\hbar}(\Phi)$ is a DG $\hbar$-algebra with $\mathbb{C}[[\hbar]]$-linear differential, of degree -1 ; moreover, $\mathfrak{D}_{\hbar}(\Phi)_{0}=F_{\hbar}$ and $\mathfrak{A}_{\hbar}(\Phi)=H^{0}\left(\mathfrak{D}_{\hbar}(\Phi)\right)$. There are natural ' $\hbar$-analogues' of properties (7.1.1)-7.1.4).

Corollary 7.2.4. Let $\Phi$ be a potential as in (7.2.3. Then:

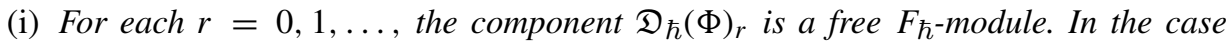
where all $\Phi_{j}$ are homogeneous of the same degree $d$, the homogeneous component $\mathfrak{D}^{(s)}(\Phi)_{r}$ is a finite rank free $\mathbb{C}[[\hbar]]$-module, for any $s \geq 0$.

(ii) Reduction modulo $\hbar$ induces a DG algebra isomorphism $\mathfrak{D}\left(\Phi_{0}\right) \stackrel{\sim}{\rightarrow} \mathfrak{D}_{\hbar}(\Phi) / \hbar$. $\mathfrak{D}_{\hbar}(\Phi)$ which, in the homogeneous case, is compatible with the weight gradings on each side.

Proof. Part (i) follows from an ' $\hbar$-analogue' of property (7.1.2); part (ii) follows from definitions.

\subsection{Formal deformations of potentials}

For any vector space, resp. algebra, $C$ let $C((\hbar))$ be the vector space, resp. algebra, of formal Laurent series with coefficients in $C$. In particular, we put $\mathbb{k}=\mathbb{C}((\hbar))$, the field of Laurent series.

It is clear that $\mathbb{k} \otimes_{\mathbb{C}[[\hbar]]} F_{\hbar}=F((\hbar))$. Moreover, $F_{\text {cyc }}[[\hbar]] \subset F_{\text {cyc }}((\hbar))=[F((\hbar))]_{\text {cyc }}$. Therefore, any potential $\Phi \in F_{\text {cyc }}[[\hbar]]$ may also be viewed as a potential for the $\mathbb{k}$ algebra $F((\hbar))$. Thus, one may view $\mathbb{k}$ as a ground field and form a $\mathbb{k}$-algebra $\mathfrak{A}(\Phi)=$ $\left.F((\hbar)) /\left\langle\partial_{j} \Phi\right\rangle\right\rangle_{j=1, \ldots, n}$. To emphasize the fact that the latter is a $\mathbb{k}$-algebra, we will write $\mathfrak{A}_{\mathbb{k}}(\Phi):=\mathfrak{A}(\Phi)$. There is an obvious $\mathbb{k}$-algebra isomorphism $\mathfrak{A}_{\mathbb{k}}(\Phi)=\mathbb{k} \otimes_{\mathbb{C}[[\hbar]]} \mathfrak{A}_{\hbar}(\Phi)$.

We begin with the following result which says that being a CY-potential is an 'open condition'.

Proposition 7.3.1. Fix a homogeneous $C Y$-potential $\Phi_{0} \in F_{\text {cyc }}^{(d)}$.

(i) For any (not necessarily homogeneous) element $\Phi^{\prime} \in F_{\text {cyc }}[[\hbar]]$, the sum $\Phi=\Phi_{0}+$ $\hbar \cdot \Phi^{\prime}$ is a Calabi-Yau potential for the algebra $F((\hbar))$. Furthermore, $\mathfrak{A}_{\hbar}(\Phi)$ is a flat $\hbar$-algebra and the natural projection yields an algebra isomorphism

$$
\mathfrak{A}\left(\Phi_{0}\right) \stackrel{\sim}{\rightarrow} \mathfrak{A}_{\hbar}(\Phi) / \hbar \cdot \mathfrak{A}_{\hbar}(\Phi) .
$$

(ii) For any element $\Phi^{\prime} \in F_{\text {cyc }}^{<d}$, the sum $\Phi=\Phi_{0}+\Phi^{\prime}$ is a CY-potential for the algebra $F$. Furthermore, the natural projection yields a graded algebra isomorphism

$$
\mathfrak{A}\left(\Phi_{0}\right) \stackrel{\sim}{\rightarrow} \operatorname{gr} \mathfrak{A}(\Phi) .
$$

We remark that part (ii) of Proposition 7.3.1 is due to Berger and Taillefer [BT]; cf. also [Gi, Corollary 5.4.4] for an alternative approach.

Proof of Proposition 7.3.1. To prove (i), let $K:=\mathfrak{D}_{\hbar}(\Phi)$. Corollary 7.2.4(i) ensures that the assumptions of Lemma 7.2.1 hold for $K$. It follows from property (7.1.4) and Lemma 
7.2.1(i) that the DG algebra $\mathfrak{D}_{\hbar}(\Phi)$ is acyclic in positive degrees. Hence, the DG algebra $\mathfrak{D}_{\mathbb{k}}(\Phi)=\mathbb{k} \otimes_{\mathbb{C}[[\hbar]]} \mathfrak{D}_{\hbar}(\Phi)$ is acyclic in positive degrees as well. Thus, property (7.1.3) implies that $\Phi$ is a Calabi-Yau potential. Now, Lemma 7.2.1 (ii) ensures that $\mathfrak{A}_{\hbar}(\Phi)$ is a flat $\hbar$-algebra, and Lemma 7.2.1 (iii) completes the proof of Proposition 7.3.1 (i).

Now, we prove part (ii) of Proposition 7.3.1 by an argument involving Rees algebras that will also be used later in this section. Let $F_{\hbar}^{\circ}:=F[\hbar]=\mathbb{C}[\hbar] \otimes F$. We assign the variable $\hbar$ degree +1 . This, together with the grading $F=\bigoplus_{s} F^{(s)}$, makes $F_{\hbar}^{\cdot}$ a graded $\mathbb{C}[\hbar]$-algebra, the Rees algebra of $F$, the latter being viewed as a filtered algebra. Thus, $\left(F_{\hbar}^{\bullet}\right)_{\text {cyc }}$ is a graded $\mathbb{C}[\hbar]$-module.

Next, write a decomposition $\Phi^{\prime}=\Phi^{(d-1)}+\Phi^{(d-2)}+\cdots+\Phi^{(0)}$ into homogeneous components $\Phi^{(r)} \in F_{\mathrm{cyc}}^{(r)}, r=1, \ldots, d$. Introduce a new homogeneous potential (of degree $d$ ) for the graded algebra $F_{\hbar}^{\cdot}=F[\hbar]$ as follows:

$$
\Phi^{\hbar}:=\Phi_{0}+\hbar \cdot \Phi^{(d-1)}+\hbar^{2} \cdot \Phi^{(d-2)}+\cdots+\hbar^{d} \cdot \Phi^{(0)} \in\left(F_{\hbar}^{\cdot}\right)_{\mathrm{cyc}}=F_{\mathrm{cyc}}[\hbar] .
$$

One has a DG algebra $\mathfrak{D}_{\hbar}^{\cdot}\left(\Phi^{\hbar}\right)=\bigoplus_{r, s \geq 0} \mathfrak{D}_{\hbar}^{(s)}\left(\Phi^{\hbar}\right)_{r}$ with differential $\mathfrak{D}_{\hbar}^{(\bullet)}\left(\Phi^{\hbar}\right)_{r} \rightarrow$ $\mathfrak{D}_{\hbar}^{(\cdot)}\left(\Phi^{\hbar}\right)_{r-1}$ defined in terms of the homogeneous potential $\Phi^{\hbar}$. For each $r \geq 0$, the component $\mathfrak{D}_{\hbar}^{(\cdot)}\left(\Phi^{\hbar}\right)_{r}$ is a free graded $\mathbb{C}[\hbar]$-module and the differential is a morphism of graded $\mathbb{C}[\hbar]$-modules. Further, we have DG algebra isomorphisms (cf. Corollary7.2.4(ii))

$$
\mathfrak{D}_{\hbar}^{\cdot}\left(\Phi^{\hbar}\right) /(\hbar-1) \cdot \mathfrak{D}_{\hbar}^{\cdot}\left(\Phi^{\hbar}\right) \stackrel{\sim}{\rightarrow} \mathfrak{D}(\Phi), \quad \text { resp. } \quad \mathfrak{D}_{\hbar}^{\cdot}\left(\Phi^{\hbar}\right) / \hbar \cdot \mathfrak{D}_{\hbar}^{\cdot}\left(\Phi^{\hbar}\right) \stackrel{\sim}{\rightarrow} \mathfrak{D}\left(\Phi_{0}\right) .
$$

Here, the DG algebra on the right is acyclic in positive cohomological degrees by (7.1.4], since $\Phi_{0}$ is a homogeneous CY-potential. Hence, the DG algebra on the left is acyclic in positive cohomological degrees, by Lemma 7.2.2. Also, from (7.3.3), we deduce

$H^{0}\left(\mathfrak{D}_{\hbar}^{\cdot}\left(\Phi^{\hbar}\right) /(\hbar-1) \cdot \mathfrak{D}_{\hbar}^{\cdot}\left(\Phi^{\hbar}\right)\right) \cong \mathfrak{A}(\Phi), \quad$ resp. $\quad H^{0}\left(\mathfrak{D}_{\hbar}^{\cdot}\left(\Phi^{\hbar}\right) / \hbar \cdot \mathfrak{D}_{\hbar}^{\cdot}\left(\Phi^{\hbar}\right)\right) \cong \mathfrak{A}\left(\Phi_{0}\right)$.

Thus, the last statement of Lemma 7.2.2 yields the algebra isomorphism $\mathfrak{A}\left(\Phi_{0}\right) \cong$ gr $\mathfrak{A}(\Phi)$.

\subsection{The case: $n=3$}

We put $F=\mathbb{C}\langle x, y, z\rangle$ and assign the generators $x, y, z$ positive weights $(a, b, c)$. Let $d:=a+b+c$.

First of all, we know that $\Phi_{0}:=x y z-y x z$ is a CY-potential of degree $d$. In other words, we have $\Phi_{0} \in \mathrm{CY}_{3}(d, a, b, c)$. We recall Definition 3.4.3 and deduce

Corollary 7.4.1. (i) A generic homogeneous potential $\Phi \in F^{(d)}$ is a CY-potential; the Hilbert-Poincaré series of the corresponding graded algebra $\mathfrak{A}(\Phi)$ is equal to that of the algebra $\mathbb{C}[x, y, z]$.

(ii) For any $\Phi^{\prime} \in F_{\text {cyc }}^{<d}$, the sum $\Phi=x y z-y x z+\Phi^{\prime}$ is a CY-potential; moreover, the natural projection yields a graded algebra isomorphism

$$
\mathbb{C}[x, y, z] \stackrel{\sim}{\rightarrow} \operatorname{gr} \mathfrak{A}(\Phi) .
$$


Proof. Part (ii) follows from Proposition 7.3.1 (ii). Further, we observe that the set $\mathrm{CY}_{3}(d, a, b, c)$ contains $\Phi_{0}$, hence is nonempty. Therefore, part (i) follows from Lemma 7.1 .5 .

Recall that $\mathbb{k}=\mathbb{C}((\hbar))$. Since $\mathrm{CY}_{3}(d, a, b, c) \neq \emptyset$ for $d=a+b+c$, from Proposition 7.3.1 (i) we deduce

Lemma 7.4.2. For any element $\Phi^{\prime} \in F_{\text {cyc }}[[\hbar]]$, the sum $\Phi=x y z-y x z+\hbar \cdot \Phi^{\prime}$ is a CY-potential for the $\mathbb{k}$-algebra $F((\hbar))$. Furthermore, the $\hbar$-algebra $\mathfrak{A}_{\hbar}(\Phi)$ with relations

$$
x y-y x=\hbar \cdot \frac{\partial \Phi^{\prime}}{\partial z}, \quad y z-z y=\hbar \cdot \frac{\partial \Phi^{\prime}}{\partial x}, \quad z x-x z=\hbar \cdot \frac{\partial \Phi^{\prime}}{\partial y}
$$

is a flat formal deformation of the polynomial algebra $\mathbb{C}[x, y, z]$.

Reducing the flat deformation of the lemma modulo $\hbar^{2}$, one obtains in a standard way a Poisson bracket on $\mathbb{C}[x, y, z]$. To describe this Poisson bracket, consider the natural abelianization map

$$
\mathbb{C}\langle x, y, z\rangle_{\text {cyc }} \rightarrow \mathbb{C}[x, y, z], \quad f \mapsto f^{\mathrm{ab}} .
$$

Further, expand the potential in Lemma 7.4 .2 as a power series in $\hbar$ and write

$$
\Phi=x y z-y x z+\hbar \cdot \Phi_{1}+\hbar^{2} \cdot \Phi_{2}+\cdots, \quad \Phi_{j} \in \mathbb{C}\langle x, y, z\rangle_{\mathrm{cyc}} .
$$

It is easy to show that the Poisson bracket on $\mathbb{C}[x, y, z]$ arising from the flat deformation of Lemma 7.4.2 is given by formula 1.3.1; specifically, we have

$$
\{-,-\}=\{-,-\}_{\phi} \quad \text { where } \quad \phi:=\left(\Phi_{1}\right)^{\mathrm{ab}} \in \mathbb{C}[x, y, z],
$$

the image under the abelianization map of the degree 1 term in the $\hbar$-power series expansion of $\Phi$.

\section{From Poisson to Hochschild cohomology}

\subsection{Deforming central elements}

We fix a triple of positive weights $(a, b, c)$. Put $F=\mathbb{C}\langle x, y, z\rangle$ and assign the generators $x, y, z$ some positive weights $a, b, c$, respectively. This gives the ascending filtration $F \leq m, m=0,1, \ldots$, on $F$, as in $\$ 3.2$ Further, we introduce a variable $\hbar$ of degree zero and use the notation $F_{\hbar}^{\leq m}:=\left(F^{\leq m}\right)[[\hbar]]$, resp. $F_{\text {cyc }}^{\leq m}[[\hbar]]$, for the corresponding induced filtrations on the $\hbar$-algebra $F_{\hbar}$, resp. $\left(F_{\hbar}\right)_{\text {cyc }}=\left(F_{\text {cyc }}\right)[[\hbar]]$. Thus, given a potential $\Phi \in\left(F_{\hbar}\right)_{\text {cyc }}^{\leq m}$, we get a filtered $\hbar$-algebra $\mathfrak{A}_{\hbar}(\Phi)$. Note that these filtrations on $F_{\hbar}$, $\left(F_{\hbar}\right)_{\text {cyc }}$, and $\mathfrak{A}_{\hbar}(\Phi)$ are not exhaustive, rather, e.g., $\bigcup_{m \geq 0} \mathfrak{A}_{\hbar}^{\leq m}(\Phi)$ is dense in $\mathfrak{A}_{\hbar}(\Phi)$ with respect to the $\hbar$-adic topology.

Now, we put $d=a+b+c$, and we recall the notation $\mathbb{k}=\mathbb{C}((\hbar))$, resp. $\mathfrak{A}_{\mathbb{k}}(\Phi)=$ $\mathbb{k} \otimes_{\mathbb{C}[[\hbar]]} \mathfrak{A}_{\hbar}(\Phi)$ and Definition 1.3 .2 
Proposition 8.1.1. (i) For any potential $\Phi \in\left(F_{\hbar}\right)_{\text {cyc }}^{\leq d}$ of the form $(7.4 .4)$, with $\Phi_{0}=$ $x y z-y x z$, the $\hbar$-algebra $\mathfrak{A}_{\hbar}(\Phi)$ contains a central element $\Psi \in \mathfrak{A}_{\hbar}^{\leq d}(\Phi)$ such that $\Psi(\bmod \hbar)=\left(\Phi_{1}\right)^{\mathrm{ab}}$.

Assume, in addition, that $\left(\Phi_{1}\right)^{\mathrm{ab}}$ is a homogeneous polynomial of degree $a+b+c$ with an isolated singularity. Then we have:

(ii) There is a bigraded $\mathbb{k}$-algebra isomorphism

$$
H H^{\bullet}\left(\mathfrak{A}_{\mathbb{k}}(\Phi)\right) \cong \mathbb{k} \otimes P H^{\bullet}\left(\mathscr{A}_{\phi}\right) \text { where } \phi:=\left(\Phi_{1}\right)^{\mathrm{ab}} \in \mathbb{C}[x, y, z] .
$$

(iii) The center of $\mathfrak{A}_{\hbar}(\Phi)$ is $Z\left(\mathfrak{A}_{\hbar}(\Phi)\right)=\mathbb{C}[\Psi][[\hbar]]$, a free topological $\hbar$-algebra in one generator, and $H H^{1}\left(\mathfrak{A}_{\mathbb{k}}(\Phi)\right)=\mathbb{k}[\Psi] \mathrm{Eu}$, is a rank one free $\mathbb{k}[\Psi]$-module generated by the Euler derivation.

Proof of Proposition 8.1.1 $(i)$. Let $R=\mathbb{C}[u]$ be a graded polynomial algebra where the variable $u$ is assigned grade degree 1 . Below, we consider $R$ as a ground ring, and write $R[x, y, z]=\mathbb{C}[x, y, z, u]$, a polynomial $R$-algebra. Given a commutative $R$-algebra $A$ we use the notation $\Omega_{R}^{\bullet} A$, resp. $\mathfrak{X}_{R}^{\bullet} A$, for the algebra of relative differential forms with respect to the subalgebra $R \subset A$, resp. $R$-linear polyderivations of $A$.

Given a filtered algebra $B$ we write $\mathrm{R} B^{\bullet}=\sum_{m \geq 0} B^{\leq m} \cdot u^{m}$ for the corresponding Rees algebra, a flat graded $R$-algebra. Thus, associated with the filtered algebra $F$, resp. $F_{\hbar}$, one has a graded $R$-algebra $\mathrm{R} F$, resp. a graded $R[[\hbar]]$-algebra $\mathrm{R} F_{\hbar}$.

Now, fix a potential $\Phi=\sum \hbar^{j} \Phi_{j} \in\left(F_{\hbar}\right)_{\mathrm{cyc}}^{\leq d}$, as in (7.4.4), and for each $j=1,2, \ldots$, write $\Phi_{j}=\Phi_{j}^{(d)}+\Phi_{j}^{(d-1)}+\cdots+\Phi_{j}^{(0)}$, where $\Phi_{j}^{(m)} \in F_{\mathrm{cyc}}^{(m)}$. We introduce a new homogeneous potential of degree $d$ similar to the one in 7.3.2 (but where the role of $\hbar$ is now played by the variable $u$ ),

$\Phi^{u}=x y z-y x z+\sum_{j=1}^{\infty} \hbar^{j} \cdot \Phi_{j}^{u} \in \mathrm{R} F_{\text {cyc }}[[\hbar]]$, where $\Phi_{j}^{u}:=\sum_{m=0}^{d} u^{m} \cdot \Phi_{j}^{(d-m)} \in \mathrm{R}^{(d)} F_{\text {cyc }}$.

Associated with the potential $\Phi$, resp. $\Phi^{u}$, we have a filtered $\hbar$-algebra $\mathfrak{A}_{\hbar}(\Phi)$, resp. graded $R[[\hbar]]$-algebra $\mathfrak{A}_{\hbar}\left(\Phi^{u}\right)$. Clearly, there is a natural graded $\hbar$-algebra isomorphism $\mathrm{RA}_{\hbar}(\Phi) \cong \mathfrak{A}_{\hbar}\left(\Phi^{u}\right)$.

One can prove $R$-analogues of Corollary 7.4.1 and of Lemma 7.4.2. This way, one deduces that the natural projection $\mathfrak{A}_{\hbar}\left(\Phi^{u}\right) / \hbar \cdot \mathfrak{A}_{\hbar}\left(\Phi^{u}\right) \stackrel{\sim}{\rightarrow} R[x, y, z]$ is a graded algebra isomorphism. Thus, the algebra $\mathfrak{A}_{\hbar}\left(\Phi^{u}\right)$ provides a $\mathbb{C}^{\times}$-equivariant flat formal deformation (where $\hbar$ is the deformation parameter and where the $\mathbb{C}^{\times}$-equivariant structure comes from the grading) of $\mathcal{R} \mathcal{A}:=R[x, y, z]$, the latter being viewed as a Poisson $R$ algebra with an $R$-bilinear Poisson bracket arising from the polynomial $\phi^{u}:=\left(\Phi_{1}^{u}\right)^{\mathrm{ab}}$ (cf. 7.4.5).

Recall next that to any formal deformation-quantization of a commutative algebra $A$ one can associate a Poisson bivector $\pi_{\hbar} \in \mathfrak{X}^{2} A[[\hbar]]$ that represents the Kontsevich class of the deformation. The Kontsevich correspondence is known to respect equivariant structures arising from a reductive group action by Poisson automorphisms. Furthermore, according to a result of Dolgushev [Do], the bivector $\pi_{\hbar}$ gives a unimodular Poisson structure if and only if the corresponding deformation-quantization gives a flat family of CY 
algebras. These results by Kontsevich and Dolgushev can be easily generalized to the setting of (flat) $R$-algebras.

Now, put $\mathcal{R} \mathcal{A}_{\hbar}:=\mathcal{R} \mathcal{A}[[\hbar]]$ and let $\pi_{\hbar} \in \mathfrak{X}_{R}^{2} \mathcal{R} \mathcal{A}_{\hbar}$ be a Poisson bivector that represents the Kontsevich class of the deformation-quantization of $\mathcal{R} \mathcal{A}$ provided by the noncommutative $R[[\hbar]]$-algebra $\mathfrak{A}_{\hbar}\left(\Phi^{u}\right)$. We know, by $R$-analogues of Corollary 7.4.1 and of Lemma 7.4.2, that this deformation is indeed a flat family of CY $R$-algebras. Therefore, we conclude that the $R[[\hbar]]$-bilinear Poisson bracket $\{-,-\}$ on $\mathcal{R} \mathcal{A}_{\hbar}$ associated with the bivector $\pi_{\hbar}$ is unimodular. Moreover, since the Kontsevich correspondence respects the $\mathbb{C}^{\times}$-equivariant structure arising from the grading on $\mathfrak{A}_{\hbar}\left(\Phi^{u}\right)$, resp. on $\mathcal{R} \mathcal{A}_{\hbar}$, we deduce that the Poisson bracket associated with the bivector $\pi_{\hbar}$ respects the grading on the algebra $\mathcal{R} \mathcal{A}_{\hbar}$, i.e. $\operatorname{deg}\{f, g\}=\operatorname{deg} f+\operatorname{deg} g$ for any homogeneous elements $f, g \in \mathcal{R} \mathcal{A}_{\hbar}$ (where $\operatorname{deg} \hbar=0$ as before).

Next, one uses an $R$-analogue of Corollary 4.3.2 (i) to show that there exists a formal series of the form $\psi=\hbar \cdot \psi_{1}+\hbar^{2} \cdot \psi_{2}+\cdots, \psi_{j} \in \mathcal{R} \mathcal{A}$, such that, in $\mathfrak{X}_{R}^{2} \mathcal{R} \mathcal{A}[[\hbar]]$, one has $\pi_{\hbar}=i_{\mathrm{d} \psi} \Upsilon$. Here, $\Upsilon \in \mathfrak{X}_{R}^{3} \mathcal{R} \mathcal{A}$ is the standard 3-vector given by formula 4.3.3. Thus, deg $\Upsilon=-(a+b+c)=-d$. It follows that each element $\psi_{j} \in \mathcal{R} \mathcal{A}^{(d)}$ must be homogeneous of degree $d$. It is also immediate from 7.4.5 that, for the first term in the expansion of $\psi$, one has

$$
\psi_{1}=\left(\Phi_{1}^{u}\right)^{\mathrm{ab}}
$$

We introduce $\mathcal{R} \mathcal{A}((\hbar))$, a commutative $R((\hbar))$-algebra. One may obviously view $\psi$ as an element of $\mathcal{R} \mathcal{A}((\hbar))$. Associated with this element, there is a Poisson $R((\hbar))$-algebra $\mathcal{R} \mathcal{A}_{\psi}$ (cf. Definition 1.3.2). Clearly, $\mathcal{R} \mathcal{A}_{\psi} \cong R((\hbar)) \otimes_{R[[\hbar]]} \mathcal{R} \mathcal{A}_{\hbar}$, and the Poisson bracket on $\mathcal{R} \mathcal{A}_{\psi}$ is nothing but the $R((\hbar))$-bilinear extension of the Poisson bracket on the $\hbar$ algebra $\mathcal{R} \mathcal{A}_{\hbar}$. Similarly, associated with the potential $\Phi^{u}$, we have an $R((\hbar))$-algebra $\mathfrak{A}\left(\Phi^{u}\right):=R((\hbar)) \otimes_{R[[\hbar]]} \mathfrak{A}_{\hbar}\left(\Phi^{u}\right)$.

At this point, one applies Kontsevich's formality theorem [K1] (cf. also [CVB]). The theorem yields a graded $R((\hbar))$-algebra isomorphism

$$
H H^{\bullet}\left(\mathfrak{A}\left(\Phi^{u}\right)\right) \cong P H^{\bullet}\left(\mathcal{R} \mathcal{A}_{\psi}\right)
$$

In degree zero, in particular, we get algebra isomorphisms $Z\left(\mathfrak{A}\left(\Phi^{u}\right)\right) \cong \mathcal{Z}\left(\mathcal{R} \mathcal{A}_{\psi}\right)=$ $R((\hbar))[\psi]$. We deduce that the center of $\mathfrak{A}\left(\Phi^{u}\right)$ is generated by a degree $d$ homogeneous element. Multiplying by a power of $\hbar$, we may assume without loss of generality that this central element has the form $1 \otimes \Psi^{u} \in R((\hbar)) \otimes_{R[[\hbar]]} \mathfrak{A}_{\hbar}^{(d)}\left(\Phi^{u}\right)$, where $\Psi^{u} \in \mathfrak{A}_{\hbar}^{(d)}\left(\Phi^{u}\right)$ is such that $\Psi^{u}(\bmod \hbar)=\psi$. Notice further that the $\hbar$-algebra $\mathfrak{A}_{\hbar}\left(\Phi^{u}\right)$ has no $\hbar$-torsion since $\Phi^{u}$ is a CY-potential (see Proposition 7.3.1(i)). It follows that the map $\mathfrak{A}_{\hbar}\left(\Phi^{u}\right) \rightarrow$ $\mathfrak{A}\left(\Phi^{u}\right), a \mapsto 1 \otimes a$, is injective and therefore $\Psi^{u}$ must be a nonzero central element of the algebra $\mathfrak{A}_{\hbar}\left(\Phi^{u}\right)$.

By construction, the original potential $\Phi$ is obtained by specializing the homogeneous potential $\Phi^{u}$ at $u=1$. Thus we see that specializing the central element $\Psi^{u}$ at $u=1$ one obtains a central element $\Psi \in \mathfrak{A}(\Phi)$, as required in the statement of Proposition 8.1.1.i). 


\subsection{Proof of Proposition 8.1.1 ii)-(iii)}

Part (ii) is also an easy consequence of the Kontsevich isomorphism 8.1.3. However, assuming the statement of part (i) holds, one can give an alternative proof of part (ii) which does not involve the formality theorem. To this end, we exploit an adaptation of an argument used by Van den Bergh in the proof of [VB2, Theorem 4.1].

Recall that $\pi=i_{\mathrm{d} \phi} \Upsilon$ (cf. (4.3.3p). First, we need the following corollary of Pichereau's results.

Lemma 8.2.1. The algebra $\mathrm{PH}^{\bullet}\left(\mathscr{A}_{\phi}\right)$ is a graded commutative algebra with generators

$$
\begin{gathered}
\phi \in P H^{0}\left(\mathscr{A}_{\phi}\right), \quad \text { eu } \in P H^{1}\left(\mathscr{A}_{\phi}\right), \quad \theta_{1}=i_{\mathrm{d} f_{1}} \Upsilon, \ldots, \theta_{\mu-1}=i_{\mathrm{d} f_{\mu-1}} \Upsilon, \\
\pi \in P H^{2}\left(\mathscr{A}_{\phi}\right), \quad \Upsilon \in P H^{3}\left(\mathscr{A}_{\phi}\right),
\end{gathered}
$$

and defining relations

$$
\text { eu } \cup \pi=d \cdot \phi \cdot \Upsilon, \quad \text { eu } \cup \Upsilon=\pi \cup \Upsilon=0, \quad \theta_{i} \cup \theta_{j}=\theta_{i} \cup \pi=0, \quad \forall i, j .
$$

Proof. For any polynomial $f \in \mathbb{C}[x, y, z]$, we have eu $\wedge i_{\mathrm{d} f} \Upsilon=\mathrm{eu}(f) \cdot \Upsilon$. Hence, we deduce eu $\wedge i_{\mathrm{d} \phi} \Upsilon=d \cdot \phi \cdot \Upsilon$. Similarly, eu $\wedge i_{\mathrm{d} f_{k}} \Upsilon=\left(\operatorname{deg} f_{k}\right) \cdot f_{k} \cdot \Upsilon$ for any $k=1, \ldots, \mu-1$. The statement readily follows from this by using the description of Poisson cohomology given in Proposition 5.4.1.

Next, we let $\mathfrak{A}_{\hbar}(\Phi) \supset \hbar \cdot \mathfrak{A}_{\hbar}(\Phi) \supset \hbar^{2} \cdot \mathfrak{A}_{\hbar}(\Phi) \supset \cdots$ be the standard $\hbar$-adic filtration. The latter may be extended in a unique way to a descending $\mathbb{Z}$-filtration on the algebra $\mathfrak{A}_{\mathbb{k}}(\Phi)$ such that multiplication by $\hbar^{-1}$ shifts the filtration by one and such that for the associated graded algebra, we have gr $\mathfrak{A}_{\mathbb{k}}(\Phi)=\mathbb{C}[x, y, z]\left[\hbar, \hbar^{-1}\right]$.

The resulting associated graded Poisson bracket on gr $\mathfrak{A}_{\mathbb{k}}(\Phi)$ is easily seen to be the $\mathbb{C}\left[\hbar, \hbar^{-1}\right]$-bilinear extension of the Poisson bracket $\{-,-\}_{\phi}$, on $\mathscr{A}_{\phi}$, where $\phi=$ $\left(\Phi_{1}\right)^{\mathrm{ab}}$. In other words, we have a Poisson $\mathbb{C}\left[\hbar, \hbar^{-1}\right]$-algebra isomorphism gr $\mathfrak{A}_{\mathbb{k}}(\Phi) \cong$ $\mathscr{A}_{\phi}\left[\hbar, \hbar^{-1}\right]$.

Associated with the above defined descending filtration on the algebra $\mathfrak{A}_{\mathbb{k}}(\Phi)$, there is a standard spectral sequence with the first term (cf. [VB2, p. 224])

$$
E_{1}=P H^{\bullet}\left(\operatorname{gr} \mathfrak{A}_{\mathbb{k}}(\Phi)\right)=\mathbb{C}\left[\hbar, \hbar^{-1}\right] \otimes P H^{\bullet}\left(\mathscr{A}_{\phi}\right) \Rightarrow \operatorname{gr} H H^{\bullet}\left(\mathfrak{A}_{\mathbb{k}}(\Phi)\right) .
$$

Following an idea of Van den Bergh, we prove

Lemma 8.2.4. Each of the elements from the set of generators of the algebra $P H^{\bullet}\left(\mathscr{A}_{\phi}\right)$ given in Lemma 8.2.1 can be lifted to an element in $H^{*}\left(\mathfrak{A}_{\mathbb{k}}(\Phi)\right)$ in such a way that analogues of relations 8.2.2) hold for the lifted elements as well.

Proof of Lemma. Set $\mathfrak{A}_{\mathbb{k}}=\mathfrak{A}_{\mathbb{k}}(\Phi)$. By Proposition 8.1.1 (i) we have $H H^{0}\left(\mathfrak{A}_{\mathbb{k}}\right)=\mathbb{k}[\Psi]$. Furthermore, the central element $\Psi \in \mathfrak{A}_{\mathbb{k}}$ provides a lift of the element $\phi \in \mathscr{A}_{\phi}$, due to equation 8.1.2. 
To lift cohomology classes of degree 3, we compare two duality isomorphisms provided by Proposition 5.1.1 (i) and by 2.1.3, respectively:

$$
\begin{aligned}
g: \mathscr{A}_{\phi} /\left\{\mathscr{A}_{\phi}, \mathscr{A}_{\phi}\right\}=P H_{0}\left(\mathscr{A}_{\phi}\right) \stackrel{\sim}{\rightarrow} P H^{3}\left(\mathscr{A}_{\phi}\right), \\
G: \mathfrak{A}_{\mathbb{k}} /\left[\mathfrak{A}_{\mathbb{k}}, \mathfrak{A}_{\mathbb{k}}\right]=H H_{0}\left(\mathfrak{A}_{\mathbb{k}}\right) \stackrel{\sim}{\rightarrow} H H^{3}\left(\mathfrak{A}_{\mathbb{k}}\right) .
\end{aligned}
$$

Observe that any element $f \in \mathscr{A}_{\phi} /\left\{\mathscr{A}_{\phi}, \mathscr{A}_{\phi}\right\}$ can be trivially lifted to an element $F \in \mathfrak{A}_{\mathbb{k}} /\left[\mathfrak{A}_{\mathbb{k}}, \mathfrak{A}_{\mathbb{k}}\right]$. It follows easily that any class of the form $g(f) \in P H^{3}\left(\mathscr{A}_{\phi}\right)$ admits a lift of the form $G(F) \in H H^{3}\left(\mathfrak{A}_{\mathbb{k}}\right)$. Further, let $\mathrm{B}(F) \in H H_{1}\left(\mathfrak{A}_{\mathbb{k}}\right)$ be the image of $F$ under the Connes differential B : $H H_{0}\left(\mathfrak{A}_{\mathbb{k}}\right) \rightarrow H H_{1}\left(\mathfrak{A}_{\mathbb{k}}\right)$. Then one shows that $G(\mathrm{~B}(F)) \in$ $H H^{2}\left(\mathfrak{A}_{\mathbb{k}}\right)$, the image of $\mathrm{B}(F)$ under the duality 2.1.3), provides a lift of the class $i_{\mathrm{d} f} \Upsilon \in$ $P H^{2}\left(\mathscr{A}_{\phi}\right)$. In particular, each of the Poisson cohomology classes $\pi=i_{\mathrm{d} \phi} \Upsilon$, resp. $\theta_{k}=$ $i_{\mathrm{d} f_{k}} \Upsilon, k=1, \ldots, \mu-1$, in $P H^{2}\left(\mathscr{A}_{\phi}\right)$ (see Lemma 8.2.1 has a lift $\Pi=G(\mathrm{~B}(\Psi)$ ), resp. $\Theta_{k}=G\left(\mathrm{~B}\left(F_{k}\right)\right)$, in $H H^{2}\left(\mathfrak{A}_{\mathbb{k}}\right)$.

Finally, one lifts the class eu $\in P H^{1}\left(\mathscr{A}_{\phi}\right)$ to the corresponding Euler derivation Eu of the graded algebra $\mathfrak{A}_{\mathbb{k}}$.

It follows from our construction that all of the relations from 8.2.2, except possibly the first one, automatically hold for the lifted elements, for degree reasons. Also the remaining relation holds for it is a formal consequence of [Gi, Theorem 3.4.3(i)] and the equation $\operatorname{Eu}(\Psi)=d \cdot \Psi$.

According to the lemma, the assignment sending our generators of the algebra $P H^{\bullet}\left(\mathscr{A}_{\phi}\right)$ to the corresponding generators of the algebra $H H^{\bullet}\left(\mathfrak{A}_{\mathbb{k}}(\Phi)\right)$ can be extended to a welldefined graded $\mathbb{k}$-algebra map $\rho: \mathbb{k} \otimes P H^{\bullet}\left(\mathscr{A}_{\phi}\right) \rightarrow H H^{\bullet}\left(\mathfrak{A}_{\mathbb{k}}(\Phi)\right)$.

We claim that the map $\rho$ is an isomorphism. To prove this, we exploit [VB2] Lemma 5.2]. That lemma, combined with our Lemma 8.2.4, implies that the spectral sequence in 8.2 .3 degenerates at $E_{1}$. We deduce that, for the filtration on $H H^{\bullet}\left(\mathfrak{A}_{\mathbb{k}}(\Phi)\right)$ coming from the spectral sequence, one has

$$
\operatorname{gr} H H^{\bullet}\left(\mathfrak{A}_{\mathbb{k}}(\Phi)\right) \cong E_{1}=\mathbb{C}\left[\hbar, \hbar^{-1}\right] \otimes P H^{\bullet}\left(\mathscr{A}_{\phi}\right) .
$$

Observe further that the lifts constructed in Lemma 8.2.4 are compatible with the filtrations involved. Moreover, each term of the filtration is complete in the $\hbar$-adic topology. This, together with isomorphism (8.2.5), immediately implies, as explained at the top of page 224 in [VB2], that the map $\rho$ must be a bijection. That completes the proof of part (ii) of Proposition 8.1.1 and, at the same time, yields part (iii) (cf. Proposition 5.4.1(i)).

\subsection{Proof of Theorems 3.3.2 and 3.4.5}

Part (i) of Theorem 3.3.2 follows directly from Corollary 7.4.1(ii) and Proposition 7.3.1(ii).

Next, we prove the existence of a central element in $\mathfrak{A}(\Phi)$ from Theorem 3.3.2 (ii) for generic potentials $\Phi \in F_{\text {cyc }}^{\leq d}$, where $d=a+b+c$. To this end, one may replace the ground field $\mathbb{C}$ by a larger field and follow the strategy of Van den Bergh [VB2, §5]. Thus, we let our ground field be of the form $K((\hbar))$ for a certain field $K$. 
We assume (as we may) that the coefficitients in the expansion of $\Phi$ as a linear combination of cyclic monomials in $x, y, z$ are algebraically independent over $\mathbb{Q}$. Then, following [VB2, §5], we may assume that the potential has the form $\Phi=x y z-y x z+$ $\sum_{j>0} \hbar^{j} \cdot \Phi_{j}$, where $\Phi_{j} \in F_{\text {cyc }}^{\leq d}$. In such a case, Proposition 8.1.1 (i) ensures the existence of a central element $\Psi \in \mathfrak{A} \leq d(\Phi)$, and we are done.

The proof of part (ii) of Theorem 3.3.2 in the general case is based on a continuity argument. We will use the same notation concerning Rees algebras as in the proof of Proposition 8.1.1. (i).

Thus, given a potential $\Phi=\Phi^{(d)}+\Phi^{(d-1)}+\cdots+\Phi^{(0)}$ of degree $\leq d$, we replace it by a degree $d$ homogeneous potential $\Phi^{u}=\Phi^{(d)}+u \cdot \Phi^{(d-1)}+\cdots+\bar{u}^{d} \cdot \Phi^{(0)} \in \mathrm{R} F_{\text {cyc }}$, where $\operatorname{deg} u=1$. Further, given $\widetilde{\Psi}^{u} \in \mathrm{R} F^{(d)}$ let $\Psi^{u} \in \mathfrak{A}\left(\Phi^{u}\right)$ denote the image of $\widetilde{\Psi}$ under the projection $\mathrm{R} F^{(d)} \rightarrow \mathfrak{A}^{(d)}\left(\Phi^{u}\right)$. The condition that $\Psi^{u} \in \mathfrak{A}\left(\Phi^{u}\right)$ be a central element of the algebra $\mathfrak{A}\left(\Phi^{u}\right)$ amounts to the following three constraints on $\widetilde{\Psi}^{u}$ :

$$
v \cdot \widetilde{\Psi}^{u}-\widetilde{\Psi}^{u} \cdot v \in \partial_{\Phi}\left(\mathfrak{D}^{(d+\operatorname{deg} v)}\left(\Phi^{u}\right)_{1}\right), \quad \forall v \in\{x, y, z\}
$$

The commutator on the left is taken in the algebra $R F$, and $\partial_{\Phi}: \mathfrak{D}^{(d)}\left(\Phi^{u}\right)_{1} \rightarrow \mathfrak{D}^{(d)}\left(\Phi^{u}\right)_{0}$ $=\mathrm{R} F$ stands for the differential in the $\mathrm{DG}$ algebra $\mathfrak{D}\left(\Phi^{u}\right)$.

Let $\Xi \subset \mathrm{R} F_{\text {cyc }}^{(d)} \times \mathbb{P}\left(\mathrm{R} F^{(d)}\right)$ be the set of pairs $\left(\Phi^{u}, \mathbb{C} \cdot \widetilde{\Psi}^{u}\right)$, where $\Phi^{u} \in \mathrm{R} F_{\text {cyc }}^{(d)}$ is a homogeneous $C Y$-potential and the element $\widetilde{\Psi}^{u}$, generating the line $\mathbb{C} \cdot \widetilde{\Psi}^{u} \subset \mathrm{R} F^{(d)}$, satisfies $(8.3 .1)$. According to 7.1.6), for each $r \geq 0$, the dimension of the vector space $\partial_{\Phi}\left(\mathfrak{D}^{(r)}\left(\Phi^{u}\right)_{1}\right)$ is a (finite) integer independent of the choice of a CY-potential $\Phi^{u} \in$ $\mathrm{R} F_{\text {cyc }}^{(d)}$. It follows that the first projection $\Xi \rightarrow \mathrm{R} F_{\text {cyc }}^{(d)},\left(\Phi^{u}, \mathbb{C} \cdot \widetilde{\Psi}^{u}\right) \mapsto \Phi^{u}$, is a proper morphism. The image of this morphism is dense in $\mathrm{R} F_{\text {cyc }}^{(d)}$ since we have already established the existence of central elements in $\mathfrak{A}\left(\Phi^{\leq d}\right)$ for generic potentials. We conclude that the map $\Xi \rightarrow \mathrm{R} F_{\text {cyc }}^{(d)}$ is surjective, and our claim follows by the specialization $u \mapsto 1, \Phi^{u} \mapsto \Phi$, and $\Psi^{u} \mapsto \Psi$.

Proof of Theorem 3.4.5. Part (1) has been proved earlier, in $\$ 6.2$ To prove (2), we repeat the argument used in the proof of Theorem 3.3.2 in the case of generic potentials. This way, we see that the required statement follows from the statement of Proposition 8.1.1(iii) about the center of the algebra $\mathfrak{A}_{\mathbb{k}}(\Phi)$.

\subsection{Proof of Theorem 3.5.2}

The statement of part (i) is a graded version of the corresponding statement of Theorem 3.4.5. (1). Thus, it follows from the latter theorem.

To prove part (ii), we may again reduce the statement to the case where the ground field is $\mathbb{k}=\mathbb{C}((\hbar))$. Furthermore, we may assume the potential $\Phi$ to be of the form (7.4.4) and such that $\left(\Phi_{1}\right)^{\mathrm{ab}} \in \mathbb{C}[x, y, z]$ is a generic homogeneous polynomial of degree $d$. Our assumptions on the triple $(a, b, c)$ ensure that such a polynomial has an isolated singularity. Thus, we are in a position to apply Proposition 8.1.1.ii). The statement of Theorem 3.5.2 (ii) then follows from that proposition and from the corresponding results about Poisson cohomology proved by Pichereau and summarized in Proposition 5.4.1 
We now prove Theorem 3.5.2 (iii). We keep the above setting, in particular, we have $\mathbb{k}$ as the base field. Thus, $A=\mathfrak{A}_{\mathbb{k}}(\Phi)$ is a Calabi-Yau algebra and we know that $H H^{1}(A)=$ $\mathbb{k}[\Psi] \mathrm{Eu}$, by Proposition 8.1.1 (iii).

Let vol $\in H_{3}(A)$ denote the image of $1 \in Z(A)=H H^{0}(A)$ under the duality isomorphism 2.1.3). Then the duality gives a $\mathbb{k}[\Psi]$-module isomorphism $H H^{1}(A) \stackrel{\sim}{\rightarrow}$ $H_{2}(A)$ that sends $\mathrm{Eu} \in H H^{1}(A)$ to $i_{\mathrm{Eu}}$ vol $\in H H_{2}(A)$. Therefore, using the equation $\mathrm{Eu}(\Psi)=d \cdot \Psi$ and standard calculus identities in the framework of Hochschild cohomology (cf. [Lo, \$4.1]), for any $k \geq 0$, we compute (where dot denotes cup-product on Hochschild cohomology)

$$
\begin{aligned}
\mathrm{B}\left(\Psi^{k} \cdot i_{\mathrm{Eu}} \mathrm{vol}\right) & =\mathrm{B} \circ i_{\mathrm{Eu}}\left(\Psi^{k} \cdot \mathrm{vol}\right)=\left(\mathrm{B} \circ i_{\mathrm{Eu}}+i_{\mathrm{Eu}} \circ \mathrm{B}\right)\left(\Psi^{k} \cdot \mathrm{vol}\right) \\
& =L_{\mathrm{Eu}}\left(\Psi^{k} \cdot \mathrm{vol}\right)=k \cdot \Psi^{k-1} \cdot \mathrm{Eu}(\Psi) \cdot \mathrm{vol}+\Psi^{k} \cdot L_{\mathrm{Eu}} \mathrm{vol} \\
& =k d \cdot \Psi^{k} \cdot \mathrm{vol}+d \cdot \Psi^{k} \cdot \mathrm{vol}=(k+1) d \cdot \Psi^{k} \cdot \mathrm{vol} .
\end{aligned}
$$

Since $(k+1) d \neq 0$ for any $k=0,1, \ldots$, from the calculation above we deduce that the Connes differential gives a bijection $\mathrm{B}: H_{2}(A) \stackrel{\sim}{\rightarrow} H H_{3}(A)$. By duality, this implies that the BV-differential yields a bijection $\Delta: H H^{1}(A) \stackrel{\sim}{\rightarrow} H H^{0}(A)$. That proves one of the two isomorphisms of Theorem 3.5.2(iii).

To prove the other isomorphism, we observe that $A$ is a nonnegatively graded algebra with degree zero component equal to $\mathbb{k}$. Hence, by [EG, Lemma 3.6.1], we get an exact sequence of Hochschild homology

$$
0 \rightarrow \mathbb{k} \rightarrow H H_{0}(A) \stackrel{\mathrm{B}}{\longrightarrow} H H_{1}(A) \stackrel{\mathrm{B}}{\longrightarrow} H H_{2}(A) \stackrel{\mathrm{B}}{\longrightarrow} H H_{3}(A) \rightarrow 0 .
$$

Applying duality 2.1.3, we obtain an exact sequence of Hochschild cohomology

$$
0 \rightarrow \mathbb{k} \cdot \Upsilon \rightarrow H H^{3}(A) \stackrel{\Delta}{\longrightarrow} H H^{2}(A) \stackrel{\Delta}{\longrightarrow} H H^{1}(A) \stackrel{\Delta}{\longrightarrow} H H^{0}(A) \rightarrow 0 .
$$

We have shown earlier that the last map $\Delta$ on the right in this exact sequence is a bijection. This forces the first map $\Delta$ on the left to be a surjection, and we are done.

Remark 8.4.3. There are also Poisson cohomology counterparts of exact sequences 8.4.1 - 8.4.2). The counterpart of 8.4.1) follows, using Cartan's homotopy formula $L_{\mathrm{eu}}=\mathrm{d} \circ i_{\mathrm{eu}}+i_{\mathrm{eu}} \circ \mathrm{d}$, from the fact that the operator $L_{\mathrm{eu}}$ acts on $\Omega^{j} \mathscr{A}_{\phi}$ with positive weights, for any $j \neq 0$. The analogue of 8.4 . can be deduced from this by duality (cf. Proposition 5.1.1).

Further, an explicit description of the group $P H^{2}\left(\mathscr{A}_{\phi}\right)$ given by Pichereau $[\mathrm{P}]$ shows that the map $\delta: P H^{3}\left(\mathscr{A}_{\phi}\right) \rightarrow P H^{2}\left(\mathscr{A}_{\phi}\right)$, equivalently, the map d : $P H_{0}\left(\mathscr{A}_{\phi}\right) \rightarrow$ $P H_{1}\left(\mathscr{A}_{\phi}\right)$, is surjective as well. This, combined with spectral sequence [8.2.5], may be used to obtain an alternative proof of Theorem 3.5.2(iii). 


\subsection{Sketch of proof of Theorem 3.4.4}

We begin with part (i). First of all we introduce a space of deformation parameters similar to the one used in the proof of Theorem 2.5.3. Specifically, let $S_{\mathfrak{A}}$ be the space of tuples $(t, c, P, Q, R)$. We have $\operatorname{dim} S_{\mathfrak{A}}=(p-1)+(q-1)+(r-1)+2=\mu$, by (2.5.4).

For each $s=(t, c, P, Q, R) \in S_{\mathfrak{A}}$ we let $A_{s}:=\mathfrak{A}\left(\Phi_{P, Q, R}^{t, c}\right)$ be the corresponding algebra. This is a filtered algebra, with an associated graded algebra gr $A_{s}$. Hence there is an induced ascending filtration $\mathrm{HH}_{<m}{ }^{\circ}\left(A_{s}\right)$ on Hochschild cohomology, resp. homology, groups of $A_{s}$. Proving Theorem 3.4.4(i) amounts to showing that there exists a subset $U \subset S_{\mathfrak{A}}$, of sufficiently general parameters, such that for any $s \in U$, the Kodaira-Spencer map induces an isomorphism

$$
\mathrm{KS}_{s}: T_{s} S_{\mathfrak{A}} \stackrel{\sim}{\rightarrow} H H_{\leq 0}^{2}\left(A_{s}\right), \quad \forall s \in U\left(\subset S_{\mathfrak{A}}\right) .
$$

To this end, we first use the classification result from Theorem 3.4.5 (1). The theorem implies that for any choice of subset $F_{\text {cyc }}^{\circ} \subset F_{\text {cyc }}$, of generic potentials in the sense of Definition 3.4.3 the set $U:=\left\{(t, c, P, Q, R) \in S_{\mathfrak{A}} \mid \Phi_{P, Q, R}^{t, c} \in F_{\text {cyc }}^{\circ}\right\}$ is nonempty and, moreover, it 1s a subset of generic parameters in $S_{\mathfrak{A}}$, in the sense of Definition 3.4.3 again.

We have the following diagram (cf. 2.1.4):

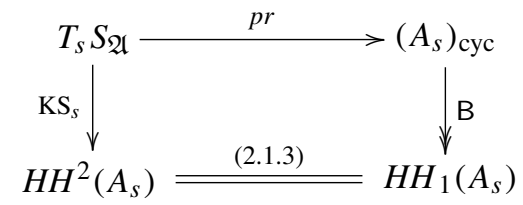

In this diagram, the map $p r$ is the tautological projection that sends a variation of the potential, viewed as an element of $\mathbb{C}\langle x, y, z\rangle_{\text {cyc }}$, to its image in $\left(A_{s}\right)_{\text {cyc. }}$ Observe further that the isomorphism 2.1.3 at the bottom of the diagram gives a bijection between $H H_{-}^{\leq d}\left(A_{S}\right)$ and $H H_{\leq 0}^{d-\bullet}\left(A_{s}\right)$. Furthermore, Proposition 2.1.5 ensures that diagram 8.5.2 commutes.

In order to prove 8.5 .1 for the algebra $A_{s}$ associated with a potential $\Phi=\Phi_{P, Q, R}^{t, c}$ with generic coefficients, we may (and will) assume that our base field is $\mathbb{k}=\mathbb{C}((\hbar)$ ) and that our potential has the form (7.4.4). We put $\phi:=\left(\Phi_{1}\right)^{\mathrm{ab}}$ (cf. 7.4 .5 ) and let $\mathscr{A}_{\phi}$ be the corresponding Poisson algebra.

There is an analogue of diagram $(8.5 .2)$ for the Poisson algebra $\mathscr{A}_{\phi}$ instead of the algebra $A_{s}$. Furthermore, there is a spectral sequence like 8.2 .5 for each of the Hochschild (co)homology groups in 8.5.2). Its $E_{1}$-term is the corresponding Poisson (co)homology group in the Poisson analogue of 8.5.2).

First of all, applying Proposition 8.1 .1 (ii) we get $\operatorname{dim} H H_{\leq 0}^{2}\left(A_{s}\right)=\operatorname{dim} P H_{\leq 0}^{2}\left(\mathscr{A}_{\phi}\right)$. Now, for any homogeneous element $f$ and $k \geq 0$, we have $\operatorname{deg}\left(\phi^{k} \cdot i_{\mathrm{d} f} \Upsilon\right)=k d+\operatorname{deg} f-$ $(a+b+c)=\operatorname{deg} f+(k-1) d$. Therefore, using Proposition 5.1.1 and the notation of Proposition 5.4.1 (ii), we find that the elements $\mathrm{d} f_{1}, \ldots, \mathrm{d} f_{\mu-1}, \mathrm{~d} \phi$ form a $\mathbb{C}$-basis of the vector space $P H_{1}^{\leq d}\left(\mathscr{A}_{\phi}\right)$. Thus, we deduce

$$
\operatorname{dim} H H_{\leq 0}^{2}\left(A_{S}\right)=\operatorname{dim} P H_{\leq 0}^{2}\left(\mathscr{A}_{\phi}\right)=\operatorname{dim} P H_{1}^{\leq d}\left(\mathscr{A}_{\phi}\right)=\mu=\operatorname{dim} S_{\mathfrak{A}} .
$$


Thus, to complete the proof of part (i) it suffices to show that the map 8.5.1 is surjective. From diagram (8.5.2), we see that this would follow provided we prove the surjectivity of the composite map B。pr: $T_{s} S_{\mathfrak{A}} \rightarrow H H_{1}^{\leq d}\left(A_{S}\right)$. Using the spectral sequence in 8.2 .5 we reduce the latter statement to proving surjectivity of a similar map $T_{s} S_{\mathfrak{A}} \rightarrow \operatorname{PH}_{1}^{\leq d}\left(\mathscr{A}_{\phi}\right)$ for Poisson algebras. But this is clear since there are obvious elements in $f_{j} \in S_{\mathfrak{A}}=\mathbb{C}^{2} \times S_{p} \times S_{q} \times S_{r}$ (cf. $\$ 2.5$, proof of Theorem 2.5.3 such that the 1 -forms $\mathrm{d} f_{1}, \ldots, \mathrm{d} f_{\mu-1}, \mathrm{~d} \phi$ give a basis of the vector space $P H_{1}^{\leq d}\left(\mathscr{A}_{\phi}\right)$.

The proof of Theorem 3.4 .5 (ii) proceeds in a similar way. We omit the details.

\section{Appendix: computer calculation}

In the $E_{6}$ case the relations in the algebra $\mathfrak{A}\left(\Phi_{P, Q, R}^{t, c}\right)$ take the following form:

$$
\begin{aligned}
& x y-q y x-t z^{2}+c_{1} z+c_{2}, \\
& y z-q z y-t x^{2}+a_{1} x+a_{2}, \\
& z x-q x z-t y^{2}+b_{1} y+b_{2} .
\end{aligned}
$$

The corresponding central element $\Psi$ was computed by Eric Rains using MAGMA. It reads

$$
\begin{aligned}
t(q+1)\left(t\left(t^{3}+1\right)\right. & \left.y^{3}+\left(q^{3}-t^{3}\right) y z x-q\left(t^{3}+1\right) z y x+t\left(q^{3}-t^{3}\right) z^{3}\right) \\
& -t\left(q^{2}+q t^{3}+q+2 t^{3}+1\right) b_{1} y^{2} \\
& +\left(q t^{3}-q^{2}\right) a_{1} y z+t^{3}(q+1) b_{1} z x+\left(q^{3}+q t^{3}\right) a_{1} z y \\
& +q(q+1) t^{3} c_{1} y x+t\left(2 q t^{3}+t^{3}-q^{4}-q^{3}-q^{2}\right) c_{1} z^{2} \\
& -\left(\left(q^{3} t+2 q^{2} t+q t\right) a_{2}+q^{2} a_{1}^{2}+q t^{2} b_{1} c_{1}\right) x \\
& -t\left(\left(q^{3} b_{2}+2 q^{2}+q t^{3}+2 q+t^{3}+1\right) b_{2}+q t a_{1} c_{1}-t^{2} b_{1}^{2}\right) y \\
& -t\left(\left(q^{4}+2 q^{3}+2 q^{2}-q t^{3}+q-t^{3}\right) c_{2}+q t^{2} c_{1}^{2}+q t a_{1} b_{1}\right) z .
\end{aligned}
$$

We refer to $[\mathbb{R}]$ for more complicated formulas in the $E_{7}$ and $E_{8}$ cases.

Remark. We were informed by the referee that such formulas were also obtained by a computer calculation in D. Stephenson's thesis.

Acknowledgments. We are grateful to Mike Artin and Eric Rains for useful discussions. We also thank Eric Rains for allowing us to reproduce his computer computations in the appendix to this paper. We are grateful to the referee for comments and references. The work of E.G. was partially supported by the NSF grant DMS-0504847. The work of V.G. was partially supported by the NSF grant DMS-0601050.

\section{References}

[A] Artin, M.: Some problems on three-dimensional graded domains. In: Representation Theory and Algebraic Geometry, London Math. Soc. Lecture Note Ser. 238, Cambridge Univ. Press, 1-19 (1997) Zbl 0888.16025 MR 1477464 
[AS] Artin, M., Schelter, W.: Graded algebras of global dimension 3. Adv. Math. 66, 171-216 (1987) Zbl 0633.16001 MR 0917738

[ATV] Artin, M., Tate, J., Van den Bergh, M.: Some algebras associated to automorphisms of elliptic curves. In: The Grothendieck Festschrift, Vol. I, Progr. Math. 86, Birkhäuser Boston, 33-85 (1990) Zbl 0744.14024 MR 1086882

[AV] Artin, M.: Van den Bergh, M.: Twisted homogeneous coordinate rings. J. Algebra 133, 249-271 (1990) Zbl 0717.14001 MR 1067406

[BT] Berger, R., Taillefer, R.: Poincaré-Birkhoff-Witt deformations of Calabi-Yau algebras. J. Noncommutative Geom. 1, 241-270 (2007) Zbl 1161.16022 MR 2308306

[Bo] Bocklandt, R.: Graded Calabi Yau algebras of dimension 3. J. Pure Appl. Algebra 212, 14-32 (2008) Zbl 1132.16017 MR 2355031

[B] Brieskorn, E.: Singular elements of semi-simple algebraic groups. In: Actes du Congrès International des Mathématiciens (Nice, 1970), Tome 2, Gauthier-Villars, Paris, 279-284 (1971) Zbl 0223.22012 MR 0437798

[BSV] Le Bruyn, L., Smith, P., Van den Bergh, M.: Central extensions of three-dimensional Artin-Schelter regular algebras. Math. Z. 222, 171-212 (1996) Zbl 0876.17019 MR 1429334

[Br] Brylinski, J.-L.: A differential complex for Poisson manifolds. J. Differential Geom. 28, 93-114 (1988) Zbl 0634.58029 MR 0950556

[CVB] Calaque, D., Van den Bergh, M.: Hochschild cohomology and Atiyah classes. Adv. Math. 224, 1839-1889 (2010) Zbl pre05729700 MR 2646112

[C1] Cassidy, T.: Global dimension 4 extensions of Artin-Schelter regular algebras. J. Algebra 220, 225-254 (1999) Zbl 0942.16050 MR 1713429

[C2] Cassidy, T.: Central extensions of Stephenson's algebras. Comm. Algebra 31, 1615-1632 (2003) Zbl 1061.16045 MR 1972883

[CK] Chan, D., Kulkarni, R.: Del Pezzo orders on projective surfaces. Adv. Math. 173, 144-177 (2003) Zbl 1051.14005 MR 1954458

[CBEG] Crawley-Boevey, W., Etingof, P., Ginzburg, V.: Noncommutative geometry and quiver algebras. Adv. Math. 209, 274-336 (2007) Zbl 1111.53066 MR 2294224

[D] Demazure, M., et al. (eds.): Séminaire sur les Singularités des Surfaces, 1976-1977. Lecture Notes in Math. 777, Springer (1980)

[Do] Dolgushev, V.: The Van den Bergh duality and the modular symmetry of a Poisson variety. Selecta Math. (N.S.) 14, 199-228 (2009) Zbl 1172.53054 MR 2480714

[Ei] Eisenbud, D.: Homological algebra on a complete intersection, with an application to group representations. Trans. Amer. Math. Soc. 260, 35-64 (1980) Zbl 0444.13006 MR 0570778

[EG] Etingof, P., Ginzburg, V.: Noncommutative complete intersections and matrix integrals. Pure Appl. Math. Quart. 3, 107-151 (2007) Zbl 1151.14006 MR 2330156

[EOR] Etingof, P., Oblomkov, A., Rains, E.: Generalized double affine Hecke algebras of rank 1 and quantized del Pezzo surfaces. Adv. Math. 212, 749-796 (2007) Zbl 1118.14003 MR 2329319

[Gi] Ginzburg, V.: Calabi-Yau algebras. arXiv:math.AG/0612139

[GK] Ginzburg, V., Kaledin, D.: Poisson deformations of symplectic quotient singularities. Adv. Math. 186, 1-57 (2004) Zbl 1062.53074 MR 2065506

[KST] Kajiura, H., Saito, K., Takahashi, A.: Matrix factorizations and representations of quivers II: type ADE case. Adv. Math. 211, 327-362 (2007) Zbl 1167.16011 MR 2313537

[Ka] Kaledin, D.: On the coordinate ring of a projective Poisson scheme. Math. Res. Lett. 13, 99-107 (2006) Zbl 1090.53064 MR 2200049 
[K1] Kontsevich, M.: Deformation quantization of Poisson manifolds. Lett. Math. Phys. 66, 157-216 (2003) Zbl 1058.53065 MR 2062626

[K2] Kontsevich, M.: Deformation quantization of algebraic varieties. Lett. Math. Phys. 56, 271-294 (2001) Zbl 1081.14500 MR 1855264

[LR] Lago, A., Rodicio, A.: Generalized Koszul complexes and Hochschild (co-)homology of complete intersections. Invent. Math. 107, 433-446 (1992) Zbl 0768.14007 MR 1144431

[LPP] Laza, R.: Pfister, G., Popescu, D.: Maximal Cohen-Macaulay modules over the cone of an elliptic curve. J. Algebra 253, 209-236 (2002) Zbl 1056.14048 $\mid$ MR 1929188

[Le] Levasseur, T.: Some properties of noncommutative regular graded rings. Glasgow Math. J. 34, 277-300 (1992) Zbl 0824.16032 MR 1181768

[Lo] Loday, J.-L.: Cyclic Homology. Grundlehren Math. Wiss. 301, Springer (1998) Zbl 0885.18007 MR 1600246

[Ma] Marconnet, N.: Homologies of cubic Artin-Schelter regular algebras. J. Algebra 278, 638-665 (2004) Zbl 1067.53064 MR 2071658

[NVB] de Naeghel, K., Van den Bergh, M.: Ideal classes of three-dimensional Sklyanin algebras. J. Algebra 276, 515-551 (2004) Zbl 1064.16028 MR 2058456

[Or1] Orlov, D.: Derived categories of coherent sheaves and triangulated categories of singularities. In: Algebra, Arithmetic, and Geometry: in honor of Yu. I. Manin, Vol. II, Progr. Math. 270, Birkhäuser, 503-531 (2009) MR 2641200

[Or2] Orlov, D.: Triangulated categories of singularities and D-branes in Landau-Ginzburg models. Proc. Steklov Inst. Math. 246, 227-248 (2004) Zbl 1101.81093 MR 2101296

[P] Pichereau, A.: Poisson (co)homology and isolated singularities. J. Algebra 299, 747-777 (2006); cf. also C. R. Math. Acad. Sci. Paris 340, 151-154 (2005) Zbl 1113.17009 Zbl 1070.53051 MR 2228339 MR 2116774

[R] Rains, E.: Computer calculation available at http://www-math.mit.edu/ etingof/delpezzocenter

[Sa] Saito, K.: Regular system of weights and associated singularities. In: Complex Analytic Singularities, Adv. Stud. Pure Math. 8, North-Holland, Amsterdam, 479-526 (1987) Zbl 0626.14028 MR 0894306

[St1] Stephenson, D. R.: Algebras associated to elliptic curves. Trans. Amer. Math. Soc. 349, 2317-2340 (1997) Zbl 0868.16028 MR 1390046

[St2] Stephenson, D. R.: Artin-Schelter regular algebras of global dimension three. PhD Thesis, University Microfilms International (1994)

[St3] Stephenson, D. R.: Artin-Schelter regular algebras of global dimension three. J. Algebra 183, 55-73 (1996) Zbl 0868.16027 MR 1397387

[VB1] Van den Bergh, M.: A relation between Hochschild homology and cohomology for Gorenstein rings. Proc. Amer. Math. Soc. 126, 1345-1348 (1998); Erratum, 130, 2809-2810 (2002) Zbl 0894.16005 MR 1443171 MR 1900889

[VB2] Van den Bergh, M.: Noncommutative homology of some three-dimensional quantum spaces. $K$-Theory 8, 213-230 (1994) Zbl 0814.16006 MR 1291019

[VB3] Van den Bergh, M.: Blowing up of non-commutative smooth surfaces. Mem. Amer. Math. Soc. 154, no. 734 (2001) Zbl 0998.14002 MR 1846352

[Xu $\mathrm{Xu}, \mathrm{P} . \mathrm{X}$ Gerstenhaber algebras and BV-algebras in Poisson geometry. Comm. Math. Phys. 200, 545-560 (1999) Zbl 0941.17016 MR 1675117 